\title{
A Nonlinear Theory of Atmospheric Blocking: A Potential Vorticity Gradient View
}

\author{
DeHAi LuO, WenQi ZHANG, AND LinHAO ZhONG \\ Key Laboratory of Regional Climate-Environment for Temperate East Asia, Institute of \\ Atmospheric Physics, Chinese Academy of Science, and University of Chinese \\ Academy of Sciences, Beijing, China \\ Aiguo DAI \\ Department of Atmospheric and Environmental Sciences, University at Albany, \\ State University of New York, Albany, New York
}

(Manuscript received 28 October 2018, in final form 14 April 2019)

\begin{abstract}
In this paper, an extended nonlinear multiscale interaction model of blocking events in the equivalent barotropic atmosphere is used to investigate the effect of a slowly varying zonal wind in the meridional direction on dipole blocking that is regarded as a nonlinear Rossby wave packet. It is shown that the meridional gradient of potential vorticity $\left(\mathrm{PV}_{y}=\partial \mathrm{PV} / \partial y\right)$ prior to the blocking onset, which is related to the background zonal wind and its nonuniform meridional shear, can significantly affect the lifetime, intensity, and north-south asymmetry of dipole blocking, while the blocking dipole itself is driven by preexisting incident synoptic-scale eddies. The magnitude of the background $\mathrm{PV}_{y}$ determines the energy dispersion and nonlinearity of blocking. It is revealed that a small background $\mathrm{PV}_{y}$ is a prerequisite for strong and long-lived eddy-driven blocking that behaves as a persistent meandering westerly jet stream, while the blocking establishment further reduces the $\mathrm{PV}_{y}$ within the blocking region, resulting in a positive feedback between blocking and $\mathrm{PV}_{y}$. When the core of the background westerly jet shifts from higher to lower latitudes, the blocking shows a northwest-southeast-oriented dipole with a strong anticyclonic anomaly to the northwest and a weak cyclonic anomaly to the southeast as its northern pole moves westward more rapidly and has weaker energy dispersion and stronger nonlinearity than its southern pole because of the smaller $\mathrm{PV}_{y}$ in higher latitudes. The opposite is true when the background jet shifts toward higher latitudes. The asymmetry of dipole blocking vanishes when the background jet shows a symmetric double-peak structure. Thus, a small prior $\mathrm{PV}_{y}$ is a favorable precursor for the occurrence of long-lived and large-amplitude blocking.
\end{abstract}

\section{Introduction}

Atmospheric blocking is a quasi-stationary large-scale dipole structure in the geopotential height anomaly field that usually occurs over mid- to high latitudes and lasts for 10-20 days (Yeh 1949; Berggren et al. 1949; Rex 1950; Dole and Gordon 1983; Shukla and Mo 1983; Luo 2000, 2005; Diao et al. 2006; Yao et al. 2017). Such a blocking flow often behaves as a large meandering of midlatitude westerly jet streams in the unfiltered geopotential height field (Berggren et al. 1949). The time evolution structure of this kind of blocking was first obtained theoretically by Luo $(2000,2005)$, Luo and Li (2000), and Luo et al. (2014). The study on the formation

\footnotetext{
Corresponding author: Dr. Dehai Luo,ldh@mail.iap.ac.cn
}

of blocking and its variability has been an important topic since the 1950s (Yeh 1949; Charney and DeVore 1979; McWilliams 1980; Lejenäs and Økland 1983; Shutts 1983; Haines and Marshall 1987: Luo 2000, 2005; Luo et al. 2014; Nakamura and Huang 2018), because it can significantly affect local weather and climates in mid- to high latitudes, for example, often leading to cold extremes in winter and heat waves in summer (Dole et al. 2011; Kitano and Yamada 2016).

Earlier studies have suggested that traveling synopticscale eddies and large-scale topography play important roles in the formation and maintenance of atmospheric

Publisher's Note: This article was revised on 1 August 2019 to correct three instances of the form of the variable $\varphi$, and revised again on 14 August 2019 to correct a typographical error in Table 1. 

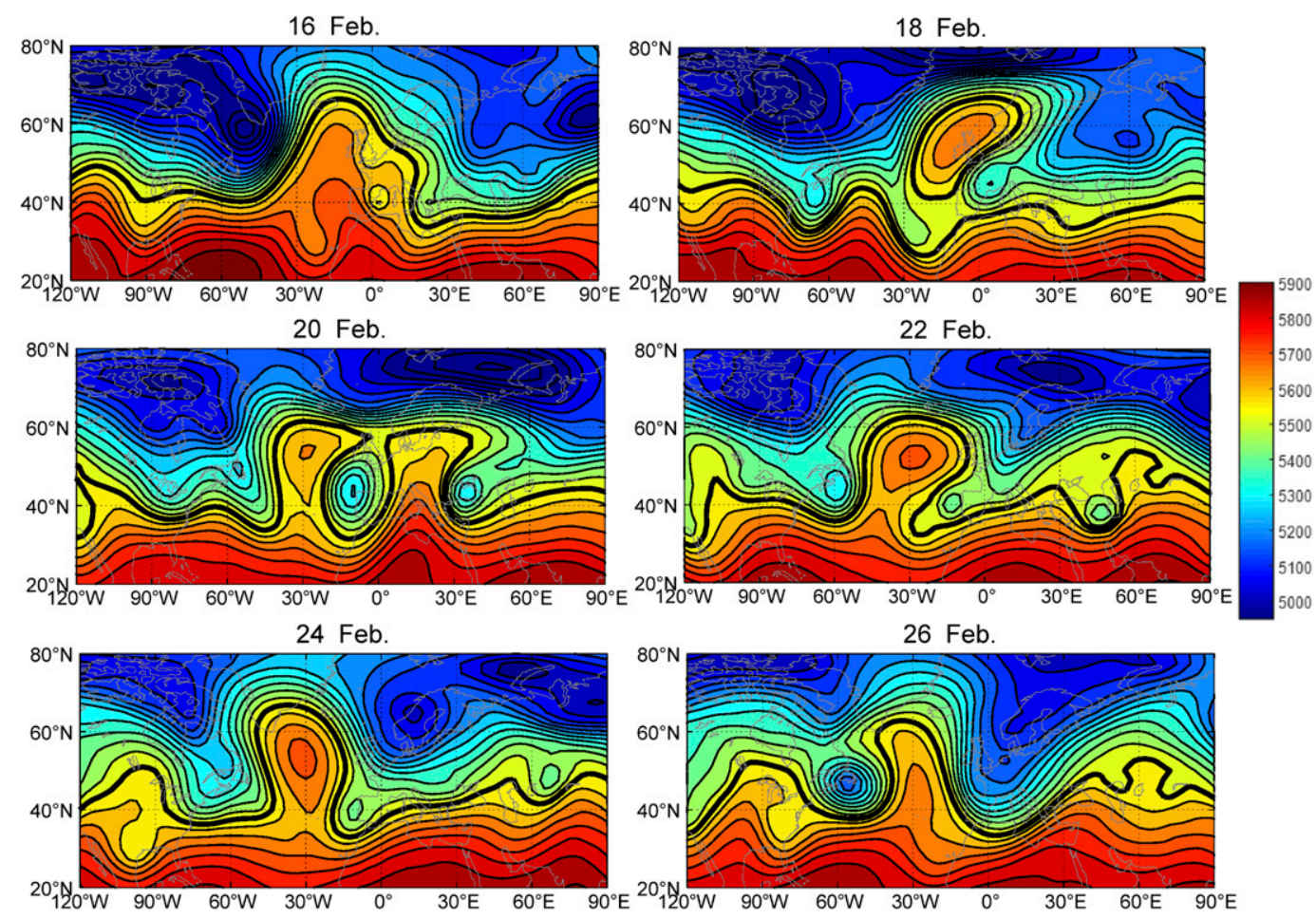

FIG. 1. Time sequences of instantaneous daily 500-hPa geopotential height fields [contour interval $(\mathrm{CI})=50 \mathrm{gpm}$ ] of a blocking event occurring in the North Atlantic from 16 Feb to 26 Feb 2004 from the European Centre of Medium-Range Weather Forecasts interim reanalysis (ERA-Interim) data on a $2.5^{\circ} \times 2.5^{\circ}$ grid. The thick black line represents the contour line of $5550 \mathrm{gpm}$ and characterizes the large meandering of a westerly jet stream comprising several anticyclones and cyclones within the blocking region.

blocking (Berggren et al. 1949; Egger 1978; Charney and DeVore 1979; Tung and Lindzen 1979; Shutts 1983; Illari and Marshall 1983; Holopainen and Fortelius 1987; Mullen 1987). However, the numerical experiment of Ji and Tibaldi (1983) indicated that the forcing of large-scale topography appears to play a secondary role in the formation of blocking compared to traveling synoptic-scale eddies. It has been recognized that the dipole blocking mainly occurs downstream of the storm track in the Pacific or Atlantic basin (Colucci 1985; Holopainen and Fortelius 1987; Mullen 1987), which also suggests that synoptic-scale eddies likely contribute to the formation and maintenance of the dipole blocking downstream of the storm tracks (Illari and Marshall 1983; Shutts 1983; Colucci 1985; Nakamura and Wallace 1993).

While many theoretical models have been proposed to explain how atmospheric blocking is maintained (Charney and DeVore 1979; McWilliams 1980; Haines and Marshall 1987; Haines and Holland 1998), they are unable to depict the life cycle of a blocking event as observed in the North Atlantic (Fig. 1) and tell us how synoptic-scale eddies drive the blocking evolution (growth and decay), as well as how the blocking and synoptic-scale eddies interact to produce the eddy deformation such as eddy straining, eddy merging, and cyclonic wave breaking (CWB). The pronounced features of the blocking evolution in the instantaneous unfiltered height field (Fig. 1) are that the synoptic-scale ridges (troughs) over the blocking region and its two sides are intensified (deepened) and shifted northward (southward) so that the Arctic cold air intrudes into lower latitudes on the eastern side and subtropical warm air enter higher latitudes on the western side. Such a behavior is the so-called CWB. During the intensification process of this North Atlantic blocking event, a large meandering of westerly jet streams can be seen in the daily unfiltered height field (thick black line in Fig. 1). These features cannot be explained using the theoretical models of Charney and DeVore (1979), McWilliams (1980), and Shutts (1983). In the previous diagnostic and theoretical models, the role of traveling synoptic-scale eddies in the blocking maintenance was considered as a time-mean effect (Shutts 1983; Hoskins et al. 1983; Haines and Marshall 1987; Haines and Holland 1998). Such a treatment excludes the instantaneous contribution of traveling synoptic-scale eddies to the blocking evolution and the feedback of an intensified blocking on the eddy evolution. It also makes it difficult 
to examine what role the synoptic-scale eddies play in the growth and decay of a dipole blocking and to explain why the blocking flow behaves as a large westerly jet meandering. Thus, the instantaneous effect of synoptic-scale eddies should be included in any theoretical model intended to study how synoptic-scale eddies reinforce and maintain a blocking dipole and how synoptic-scale eddies deform as a result of the feedback of the intensified blocking.

In recent decades, Luo and his collaborators have established a nonlinear multiscale interaction (NMI) model to elucidate how preexisting synoptic-scale eddies contribute to the evolution (growth and decay) of a downstream dipole blocking based on a zonal scale separation assumption (Luo 2000, 2005; Luo and Li 2000; Luo et al. 2001, 2014). Different from previous theoretical models, the most important advantage of this NMI model is that it shows how the life cycle of a dipole blocking with a 10-20-day time scale as a nonlinear evolution of a large-scale Rossby wave packet is generated by preexisting synoptic-scale eddies and how preexisting synopticscale eddies undergo a deformation due to the feedback of the intensified blocking (Luo 2000, 2005; Luo et al. 2014). In particular, this model can show that the intensification of blocking is followed by the northward (southward) migration of amplified small-scale ridges (troughs) and characterized by an enhanced westerly jet meandering. These features also reflect the appearance of both the eddy straining and CBW and are consistent with reanalysis data (Fig. 1). Another advantage of this theoretical model is that the preexisting incoming synoptic-scale eddies need to "match" the preexisting small block in order for it to grow, whereas the eddy straining and CWB are only a concomitant phenomenon of the blocking occurrence and secondary or not important for the blocking intensification and decay (Luo et al. 2014). This is at odds with the previous viewpoint that the eddy straining or CWB related to deformed eddies was understood as leading to the blocking onset, formation, and maintenance. In this model, the preexisting incoming synoptic-scale eddies have been shown to play a pivotal role in the life cycle (intensification, maintenance, and decay) of downstream blocking.

Atmospheric reanalysis data further revealed that the composite daily 500-hPa geopotential height anomaly field of blocking events over the North Atlantic shows a strong north-south asymmetry with a strong anticyclonic anomaly to the northwest and a weak cyclonic anomaly to the southeast, with the blocking dipole moving westward (i.e., retrogression; Fig. 2a). Such asymmetry was also seen in blocking occurring over Eurasia (Luo et al. 2016; Yao et al. 2017). Because the winter-mean zonal wind is generally stronger in midlatitudes than in high latitudes (Fig. 2b), the meridional asymmetry and the northwest-southeast
(NW-SE) orientation of the blocking dipole may be related to the meridional distribution of background westerly winds in the mid- to high latitudes. However, it is unclear what physical mechanism causes this meridional asymmetry of blocking, or how the meridional wind shear leads to this blocking asymmetry. Previous theoretical studies, including Luo et al. (2014), have not attempted to address what determines the duration and spatial shape of a blocking event, which have major implications for its impact on local weather. In particular, no theoretical studies have investigated how a changing climate, such as fast warming high latitudes, may affect the duration, intensity, location, and spatial shape of atmospheric blocking. This has become a concern because weakened zonal winds in mid- to high latitudes can occur as result of reduced meridional temperature gradients under enhanced Arctic warming (Luo et al. 2016; Yao et al. 2017; Dai et al. 2019). While the extended NMI model of Luo et al. (2018) has this ability, this model was not applied to study the above problems. The main purpose of this study is to use the extended nonlinear theoretical model of Luo et al. $(2014,2018)$ to address these issues.

This paper is organized as follows. In section 2, we describe an extended nonlinear multiscale interaction (or extended NMI) model by including a slowly varying background zonal wind. The impact of meridionally varying background zonal winds on the persistence and meridional asymmetry of an eddy-driven dipole blocking is examined in sections 3 and 4, respectively. In section 5, we discuss the impacts of the strength of the background zonal wind and its nonuniform meridional shear on the blocking dipole. The conclusions and discussion are given in section 6 .

\section{Extended nonlinear multiscale interaction model with a slowly varying zonal flow}

In our previous NMI model, the basic zonal flow is assumed to be uniform (Luo 2000, 2005; Luo and Li 2000; Luo et al. 2014). However, reanalysis data show that the basic zonal wind is slowly varying in the meridional direction (Fig. 2b). In this case, the NMI model cannot be used to examine how the slowly varying basic zonal flow affects the blocking evolution and its spatial structure as observed in Fig. 2a. Thus, the previous NMI model needs to be further extended to include the effect of slowly varying basic flow as shown in Fig. 2b. The extended NMI model of Luo et al. (2018) provides such a possibility, but the effect of background zonal wind distributions on the blocking was not examined in Luo et al. (2018).

The appearance of atmospheric blocking often leads to the most pronounced low-frequency variability in mid- to high latitudes (Dole and Gordon 1983; Shukla 
lag -10

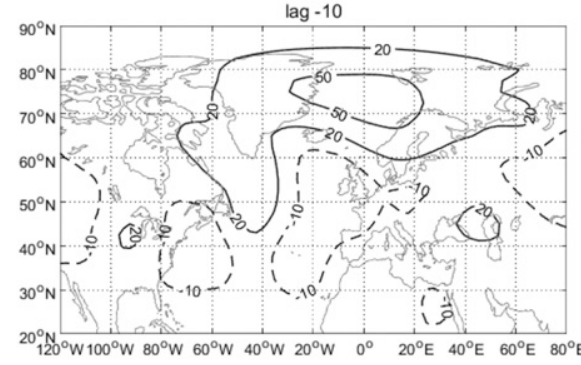

lag -6

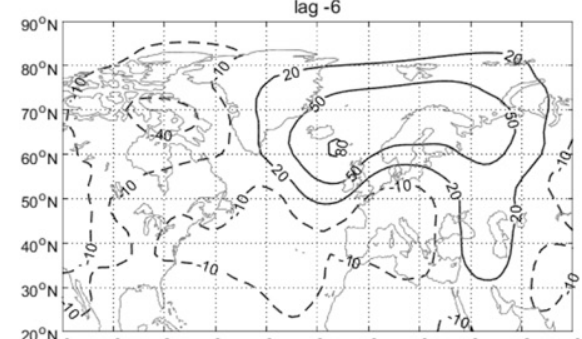

${ }_{120^{\circ} \mathrm{N}}^{\circ} \mathrm{W} 100^{\circ} \mathrm{W} 80^{\circ} \mathrm{W} 60^{\circ} \mathrm{W} \quad 40^{\circ} \mathrm{W} 20^{\circ} \mathrm{W} \quad 0^{\circ} \quad 20^{\circ} \mathrm{E} \quad 40^{\circ} \mathrm{E} \quad 60^{\circ} \mathrm{E} 80^{\circ}$

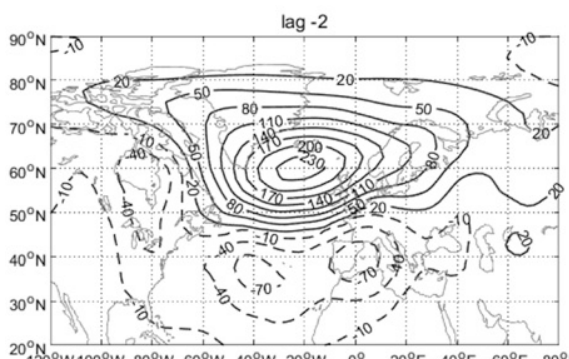

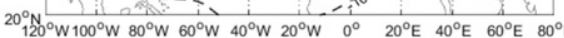

$\operatorname{lag} 2$

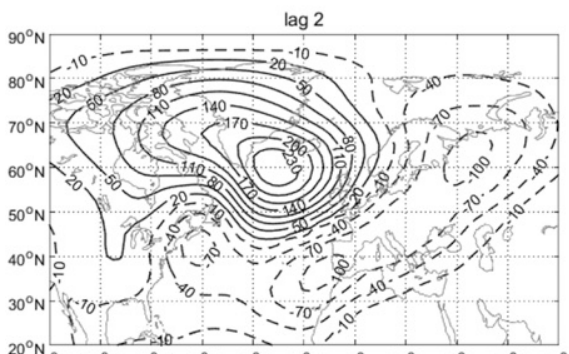

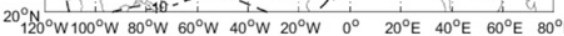

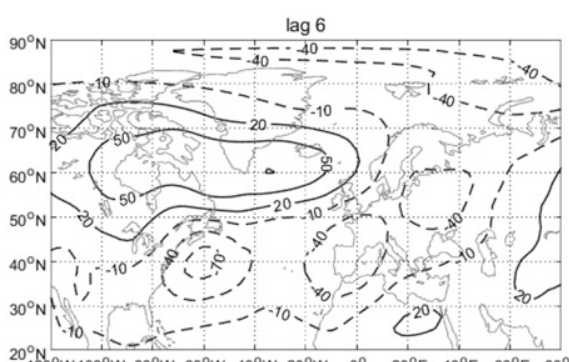

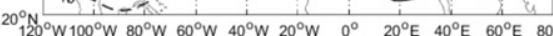

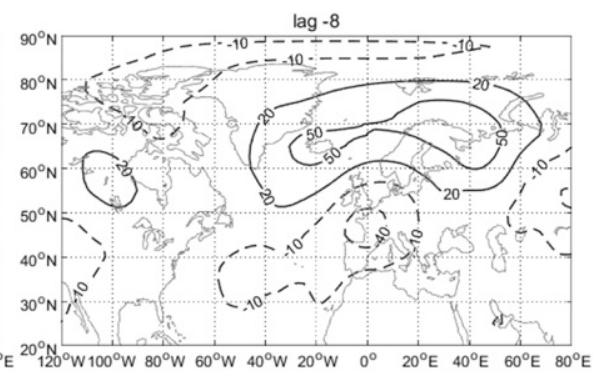

$\operatorname{lag}-4$

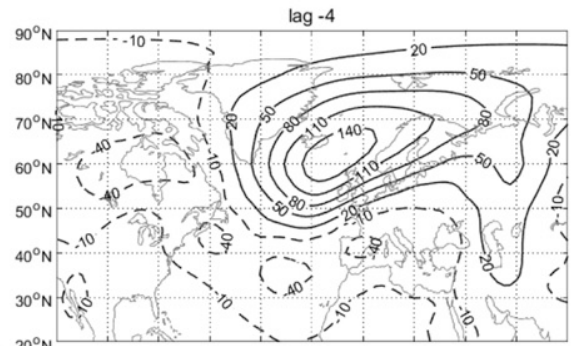

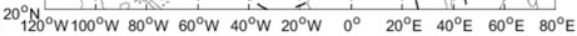
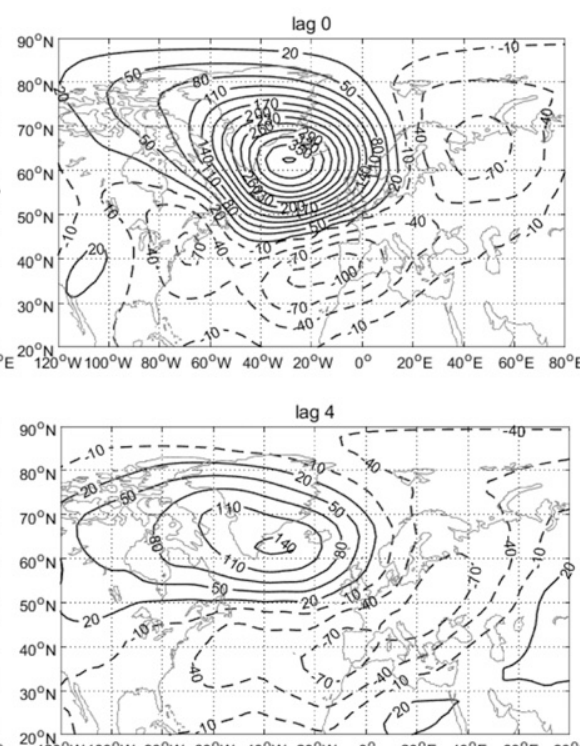

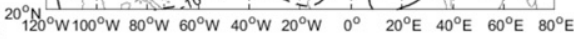

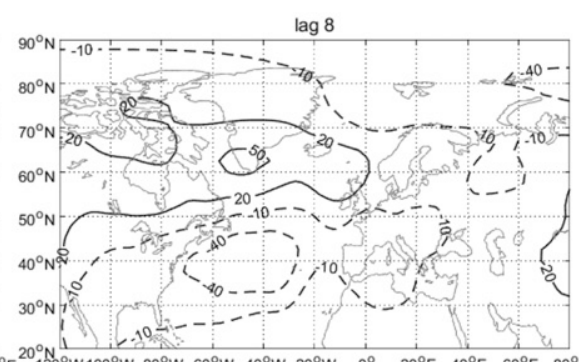

(a)

FIG. 2. (a) Time sequences of composite daily 500-hPa geopotential height (Z500) anomalies (lag 0 denotes the blocking peak day) of North Atlantic blocking events (44 cases) in winter (DJF) from December 1979-February 1980 to December 2017-February 2018 (1979-2017) identified with the Tibaldi-Molteni index with a duration threshold of 5 days (Tibaldi and Molteni 1990) and (b) DJF-mean zonal wind based on ERA-Interim data with $2.5^{\circ} \times 2.5^{\circ}$ resolution. 


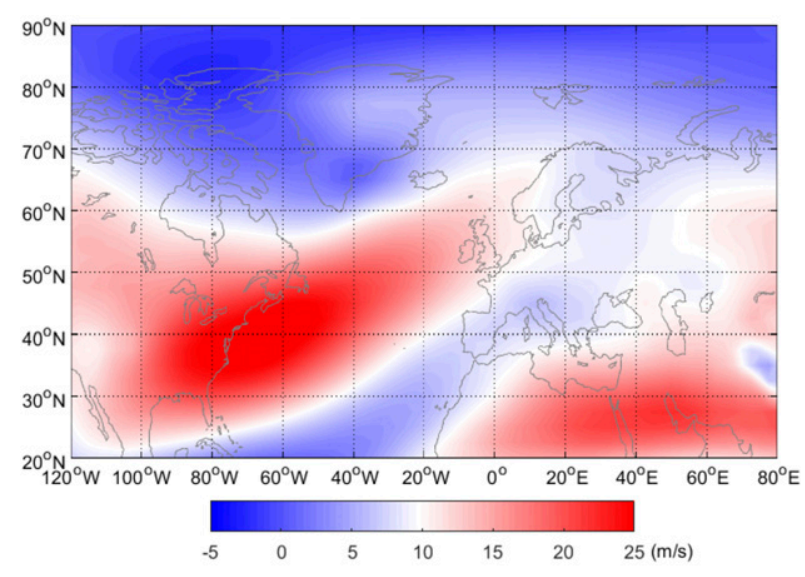

(b)

FIG. 2. (Continued)

and Mo 1983). The planetary-scale field of blocking can generally be separated into two parts: a basic flow and a planetary-scale blocking anomaly. The planetaryscale blocking anomaly is often associated with synopticscale eddies (Berggren et al. 1949; Ji and Tibaldi 1983; Shutts 1983; Holopainen and Fortelius 1987). Thus, it is reasonable that the instantaneous total blocking streamfunction field $\psi_{T}$ is decomposed into three parts of $\psi_{T}=\bar{\psi}(y)+\psi+\psi^{\prime}$ : a basic flow $\bar{\psi}(y)$, a planetary-scale blocking anomaly $\psi$, and a synoptic-scale component $\psi^{\prime}$ as in our previous studies (Luo 2000, 2005; Luo and Li 2000; Luo et al. 2014). Here, we assume that the prior basic flow is purely zonal and $\bar{\psi}(y)=-\int_{0}^{y} U\left(y^{\prime}\right) d y^{\prime}$ (the overbar is for the averaging along the latitude, $U$ is the background zonal wind prior to the blocking onset and the meridional mean wind $V \approx 0$ ). We further assume that the planetary-scale streamfunction anomaly $\psi$ has a single zonal wavenumber $k$ typically corresponding to wavenumber 2 in northern midlatitudes and the synoptic-scale eddies $\psi^{\prime}$ have large zonal wavenumbers $\tilde{k}_{j}(j=1,2,3, \ldots)$. As in Luo $(2000,2005)$, Luo and $\mathrm{Li}$ (2000), and Luo et al. (2014), when there is a scale separation assumption $k \ll \tilde{k}$, the nondimensional planetary- and synoptic-scale $\mathrm{PV}$ equations of the extended NMI (ENMI) model, scaled by characteristic length $\tilde{L}(\approx 1000 \mathrm{~km})$ and velocity $\tilde{U}\left(\approx 10 \mathrm{~m} \mathrm{~s}^{-1}\right)$, are obtained as

$$
\begin{aligned}
\left(\frac{\partial}{\partial t}+\right. & \left.U \frac{\partial}{\partial x}\right)\left(\nabla^{2} \psi-F \psi\right)+J\left(\psi, \nabla^{2} \psi\right)+\mathrm{PV}_{y} \frac{\partial \psi}{\partial x} \\
& =-\nabla \cdot\left(\mathbf{v}^{\prime} q^{\prime}\right)_{P}, \\
\left(\frac{\partial}{\partial t}+\right. & \left.U \frac{\partial}{\partial x}\right)\left(\nabla^{2} \psi^{\prime}-F \psi^{\prime}\right)+\mathrm{PV}_{y} \frac{\partial \psi^{\prime}}{\partial x} \\
& =-J\left(\psi^{\prime}, \nabla^{2} \psi\right)-J\left(\psi, \nabla^{2} \psi^{\prime}\right)+\nabla^{2} \psi_{S}^{*},
\end{aligned}
$$

where $\nabla \cdot\left(\mathbf{v}^{\prime} q^{\prime}\right)_{P}=J\left(\psi^{\prime}, \nabla^{2} \psi^{\prime}\right)_{P}$ denotes the planetaryscale component of the eddy vorticity flux divergence $\nabla \cdot\left(\mathbf{v}^{\prime} q^{\prime}\right)$ induced by synoptic eddies $\psi^{\prime}$ with its relative vorticity $q^{\prime}=\nabla^{2} \psi^{\prime}$, which has a zonal wavenumber close or identical to that of the planetary-scale blocking anomaly $\psi, \mathbf{v}^{\prime}=\left(u^{\prime}, v^{\prime}\right)=\left(-\partial \psi^{\prime} / \partial y, \partial \psi^{\prime} / \partial x\right), \mathrm{PV}=f_{0}+$ $\beta y-U_{y}-F \psi$ is the barotropic PV (Charney and DeVore 1979; McWilliams 1980) under equivalent barotropic and $V \approx 0$ conditions, $U_{y}=\partial U / \partial y, \mathrm{PV}_{y}=\partial \mathrm{PV} / \partial y=\beta-$ $U_{y y}+F U$ is the meridional background $\mathrm{PV}$ gradient, $U_{y y}=\partial^{2} U / \partial y^{2}, F=\left(\tilde{L} / R_{d}\right)^{2}, R_{d}$ is the radius of Rossby deformation, $\beta=\beta_{0} \tilde{L}^{2} / \tilde{U}, \beta_{0}$ is the meridional gradient of the Coriolis parameter at a given latitude $\varphi_{0}$. We assume $R_{d} \approx \tilde{L}$ in Eq. (1). Note that $\nabla^{2} \psi_{S}^{*}$ is the synoptic-scale vorticity source term as a synoptic-scale wavemaker that excites preexisting synoptic-scale eddies prior to the blocking onset (Luo 2005).

Because $\nabla \cdot\left(\mathbf{v}^{\prime} q^{\prime}\right)_{P}$ is time dependent, Eq. (1) can depict the life cycle of a blocking flow. In many previous studies (Shutts 1983; Haines and Marshall 1987; Haines and Holland 1998), the time-mean $\nabla \cdot \overline{\left(\mathbf{v}^{\prime} q^{\prime}\right)}$ with some large parts that mismatch the blocking component was often used, and the resulting solution could only describe the time-mean state. Thus, these earlier models overestimate the timemean effect of synoptic eddies on blocking and are not suitable for examining how a change in the background zonal wind affects the temporal evolution of blocking.

Here, we briefly describe the physical processes implied by the ENMI model regarding the generation of blocking under some simplified assumptions below. We can rewrite Eq. (1a) as $\partial q / \partial t+J(\bar{\psi}+\psi, q+$ $\mathrm{PV})=-\nabla \cdot\left(\mathbf{v}^{\prime} q^{\prime}\right)_{P}$, where $q=\nabla^{2} \psi-F \psi$. One can have $\partial q / \partial t \approx-\nabla \cdot\left(\mathbf{v}^{\prime} q^{\prime}\right)_{P}$ when the assumption $J(\bar{\psi}+\psi, q+$ $\mathrm{PV}) \approx 0$ is used as done in Luo et al. (2014). This means that the growth, maintenance, and decay of blocking is driven by the planetary-scale (blocking scale) eddy vorticity flux divergence $\nabla \cdot\left(\mathbf{v}^{\prime} q^{\prime}\right)_{P}$ induced by synopticscale eddies $\psi^{\prime}$, when this vorticity flux divergence $\left[\nabla \cdot\left(\mathbf{v}^{\prime} q^{\prime}\right)_{P}\right]$ matches the PV (i.e., $\left.q\right)$ structure of the blocking field (Luo et al. 2014). The analytical solution of the time evolution of eddy-driven blocking can be obtained from Eq. (1) by assuming $\psi^{\prime} \approx \psi_{1}^{\prime}+\psi_{2}^{\prime}$, where $\psi_{1}^{\prime}$ denotes the given preexisting incident synoptic-scale eddies upstream prior to the blocking onset and $\psi_{2}^{\prime}$ represents the deformed synoptic-scale eddies due to the feedback of intensified blocking on preexisting synoptic eddies (Luo 2000, 2005; Luo et al. 2014). In this theoretical model, deformed eddies reflect the meridional displacement of intensified synoptic-scale ridge and trough systems or eddy straining or CWB and do not play any role in the blocking growth or decay.

We can also have $\partial q / \partial t \approx-\nabla \cdot\left(\mathbf{v}_{1}^{\prime} q_{1}^{\prime}\right)_{P}$ because of $\psi_{2}^{\prime} \approx 0, \mathbf{v}^{\prime} \approx \mathbf{v}_{1}^{\prime}=\left(-\partial \psi_{1}^{\prime} / \partial y, \partial \psi_{1}^{\prime} / \partial x\right)$, and $q^{\prime} \approx q_{1}^{\prime}=\nabla^{2} \psi_{1}^{\prime}$ 
during the initial stage $(t \sim 0)$ of the blocking onset, where $-\nabla \cdot\left(\mathbf{v}_{1}^{\prime} q_{1}^{\prime}\right)_{P}$ denotes the blocking-scale eddy vorticity flux divergence induced by preexisting synoptic-scale eddies $\psi_{1}^{\prime}$ or referred to as "preexisting eddy forcing" due to preexisting eddy-eddy interaction hereafter. The relation $\partial q / \partial t \approx-\nabla \cdot\left(\mathbf{v}_{1}^{\prime} q_{1}^{\prime}\right)_{P}$ reveals that the initiation of blocking is caused by the preexisting eddy forcing $-\nabla \cdot\left(\mathbf{v}_{1}^{\prime} q_{1}^{\prime}\right)_{P}$. While intense deformed synoptic eddies $\psi_{2}^{\prime}$ can be produced by the intensified blocking, $-\nabla \cdot\left(\mathbf{v}_{1}^{\prime} q_{1}^{\prime}\right)_{P}$ is still important for the formation, maintenance, and decay of blocking (Luo 2000, 2005; Luo et al. 2014), which is also demonstrated by Mu and Jiang (2008) from a conditional nonlinear optimal perturbation view. This theory emphasizes that it does not need the eddy straining and CWB as the formation and maintenance mechanisms of blocking. Instead, this theoretical model also indicates that the eddy straining and CWB are more likely a result of the blocking formation and maintenance caused by preexisting synoptic eddies. This is different from the previous numerical results (Shutts 1983). It is further revealed that the movement, intensity, and lifetime of blocking depends not only on the duration of $-\nabla \cdot\left(\mathbf{v}_{1}^{\prime} q_{1}^{\prime}\right)_{P}$, but also on the strength of the background westerly wind that determines the energy dispersion and nonlinearity of blocking system. Below, we will use the analytical solution of the ENMI model to further demonstrate that the change of background westerly winds in strength and meridional distribution can significantly affect the persistence and meridional asymmetry of dipole blocking through changing energy dispersion and nonlinearity of the blocking system and the duration of $-\nabla \cdot\left(\mathbf{v}_{1}^{\prime} q_{1}^{\prime}\right)_{P}$.

To obtain the analytical solution of Eq. (1), we make two important assumptions: 1) the basic zonal flow is so slowly varying in the meridional direction that $U_{y}$, $U_{y y}$, and $\mathrm{PV}_{y}$ are small compared to the wavy structure of the blocking when the Wentzel-Kramers-Brillouin (WKB) method (Nayfeh 2000) is used to derive the analytical solution of Eq. (1); and 2) preexisting synoptic-scale eddies $\psi_{1}^{\prime}$ are composed of two zonal modes having a meridional monopole structure and zonal wavenumbers $\tilde{k}_{1}$ and $\tilde{k}_{2}$ with two frequencies $\tilde{\omega}_{1}$ and $\tilde{\omega}_{2}$ as a maximum simplification of preexisting incident synoptic eddies (Luo 2000, 2005; Luo et al. 2014).

We further assume that the blocking wavy anomaly has a meridional dipole structure with meridional and zonal wavenumbers $m$ and $k$, respectively. In this case, one can obtain the time-dependent solution of the eddy-driven blocking dipole in a slowly varying basic zonal flow if the envelope amplitude of the blocking wavy anomaly is also assumed to be slowly varying in the north-south direction and has slower variations compared to those in the zonal direction. These assumptions seem to be reasonable for our purpose to study the role of the meridional asymmetry of the background westerly wind in producing the meridional asymmetry of the blocking dipole because the spatial variation of observed blocking is often faster in the zonal direction than that in the meridional direction.

Similar to Luo (2000, 2005), Luo and Li (2000), and Luo et al. (2014), the asymptotic solution of the total atmospheric streamfunction of an eddy-driven blocking event derived from Eq. (1) in a slowly varying zonal flow $U(y)$ can be obtained using the WKB method. The analytical solution of Eq. (1) in a first variable form can be expressed as

$$
\begin{aligned}
\psi_{T}= & -\int_{0}^{y} U\left(y^{\prime}\right) d y^{\prime}+\psi+\psi^{\prime}=\psi_{P}+\psi^{\prime} \\
\psi_{P}= & -\int_{0}^{y} U\left(y^{\prime}\right) d y^{\prime}+\psi \approx-\int_{0}^{y} U\left(y^{\prime}\right) d y^{\prime}+\psi_{B}+\psi_{m} \\
\psi_{B}= & B \sqrt{\frac{2}{L_{y}}} \exp [i(k x-\omega t)] \sin (m y)+\mathrm{cc} \\
\psi_{m}= & -|B|^{2} \sum_{n=1}^{\infty} q_{n} g_{n} \cos (n+1 / 2) m y \\
\psi^{\prime} \approx & \psi_{1}^{\prime}+\psi_{2}^{\prime} \\
\psi_{1}^{\prime}= & f_{0}(x)\left\{\exp \left[i\left(\tilde{k}_{1} x-\tilde{\omega}_{1} t\right)\right]\right. \\
& \left.+\alpha \exp \left[i\left(\tilde{k}_{2} x-\tilde{\omega}_{2} t\right)\right]\right\} \sin \left(\frac{m}{2} y\right)+\mathrm{cc}
\end{aligned}
$$


where $\Delta k=k-\left(\tilde{k}_{2}-\tilde{k}_{1}\right)$ denotes the difference of the zonal wavenumber between the blocking wavy anomaly $\psi_{B}$ and preexisting eddy forcing $-\nabla \cdot\left(\mathbf{v}_{1}^{\prime} q_{1}^{\prime}\right)_{P}$ induced by preexisting synoptic eddies $\psi_{1}^{\prime}, \Delta \omega=\tilde{\omega}_{2}-$ $\tilde{\omega}_{1}-\omega, \omega=U k-\mathrm{PV}_{y} k /\left(k^{2}+m^{2}+F\right), \quad C_{g}=\partial \omega / \partial k=$ $U-\mathrm{PV}_{y}\left(m^{2}+F-k^{2}\right) /\left[\left(k^{2}+m^{2}+F\right)^{2}\right]$ is the group velocity, $\tilde{\omega}_{j}=U \tilde{k}_{j}-\mathrm{PV}_{y} \tilde{k}_{j} /\left(\tilde{k}_{j}^{2}+m^{2} / 4+F\right)(j=1,2)$, $|B|^{2}=B B^{*}, k=2 k_{0}, k_{0}=1 /\left(6.371 \cos \varphi_{0}\right), \lambda=\left[3\left(m^{2}+\right.\right.$ $\left.F)-k^{2}\right] \times \mathrm{PV}_{y} k /\left[\left(k^{2}+m^{2}+F\right)^{3}\right]$ is the linear dispersion term, $q_{n}=q_{N n} / \mathrm{PV}_{y}, \delta=\delta_{N} / \mathrm{PV}_{y}\left(\delta_{N}>0\right)$ is the nonlinearity strength, $m=-2 \pi / L_{y}, \alpha_{1}=1, \alpha_{2}=\alpha$, $\tilde{k}_{j}=n k_{0}+(-1)^{j} \Delta n k_{0}(j=1,2), n$ is the positive integer, $\Delta n$ is the difference between $\tilde{k}_{1}$ and $\tilde{k}_{2}$, and "cc" denotes the complex conjugate of its preceding term. Note that $\psi \approx \psi_{B}+\psi_{m}$ has been used in Eq. (2b), and it includes two parts: the blocking wavy anomaly streamfunction $\psi_{B}$ and the associated mean zonal-wind anomaly streamfunction $\psi_{m}$ (the subscript $m$ of $\psi_{m}$ denotes a zonal mean). Here, we can allow $n=10, \Delta n=1.0$, and $\alpha=-1.0$. For this case, the spatial structure of $-\nabla$. $\left(\mathbf{v}_{1}^{\prime} q_{1}^{\prime}\right)_{P}$ can match the PV of the incipient block so that the preexisting incipient synoptic-scale eddies can amplify this incipient block into a typical dipole blocking. In Eq. (2h), $G f_{0}^{2} \exp [-i(\Delta k x+\Delta \omega t)]$ has a duration of $2 \pi /(-\Delta \omega)$ and represents the role of the preexisting eddy forcing $-\nabla \cdot\left(\mathbf{v}_{1}^{\prime} q_{1}^{\prime}\right)_{P}$ in the establishment, maintenance, and decay of dipole blocking. The term $Q_{j}$ is the coefficient of the interaction between the blocking wavy anomaly and preexisting synoptic-scale eddies, $\omega$ is the frequency of the blocking wavy anomaly $\psi_{B}, \varphi_{0}$ is the reference latitude, $L_{y}$ is the width of the nondimensional beta plane channel, $f_{0}(x)=a_{0} \exp [-$ $\left.\mu \varepsilon^{2}\left(x+x_{T}\right)^{2}\right]$ is the spatial distribution of the amplitude of the preexisting synoptic-scale eddies with two frequencies $\tilde{\omega}_{j}(j=1,2)$ that is maintained by the upstream synoptic-scale wavemaker (Luo 2005), $\mu>0$, $\varepsilon \ll 1.0$ is a small parameter, and $x_{T}$ is the zonal location of the maximum eddy amplitude. In Eq. $(2 \mathrm{~h}), f_{0}(x)$ is a slowly varying function of $x$ because of $f_{0}(x)=f_{0}(\varepsilon x)(\varepsilon$ is defined as above), which means that its $x$ derivative is a higher-order small term so that the zonal variation of $f_{0}(x)$ cannot produce a lower-order meridional velocity at the boundaries. Such an assumption can avoid the boundary-generated oscillating mean flows. The terms $Q_{j}, p_{j}, r_{j}, s_{j}$, and $h_{j}$ are defined in appendix A and $\delta_{N}, g_{n}$, and $G$ are defined in appendix B. The details of the derivation of solution (2) can be found in Luo and Li (2000) and Luo (2005).

Equation (2h) can be derived after the first- and second-order solutions of Eq. (1a) are substituted into its third-order solution equation when using the WKB method. In Luo (2005), we have described how the preexisting incoming synoptic-scale eddies in the upstream side of an incipient dipole block are produced and maintained by a synoptic-scale wavemaker $\nabla^{2} \psi_{S}^{*}$ and how an incipient dipole block evolves into a typical dipole blocking under the forcing of preexisting upstream synoptic-scale eddies for $x_{T}>0$.

Equation (2d) describes the splitting of a westerly jet anomaly due to the presence of intensified dipole blocking. We also see that $B$ represents the complex envelope amplitude of the blocking wavy anomaly $\psi_{B}$ and has a complex conjugate of $B^{*}$, whose variation in time and in the zonal direction can be described by a forced nonlinear Schrödinger (NLS) Eq. (2h). Note that the blocking amplitude Eq. (2h) as a nonlinear wave packet equation includes the parameters $C_{g}, \lambda, \delta, G, \Delta k$, and $\Delta \omega$ in addition to the eddy amplitude distribution $f_{0}(x)$. Among these parameters, $G$ and $\Delta k$ are fixed constants and independent of $U$ or $\mathrm{PV}_{y}$. Thus, the blocking amplitude $B$ is dependent on the latitude because $C_{g}, \lambda, \delta$, and $\Delta \omega$ include $U(y)$ and $\mathrm{PV}_{y}$, which are the functions of $y$. To some extent, Eq. (2h) is a onedimensional equation, but essentially two-dimensional. Such an assumption may greatly simplify the complexity of the solution of our model equations. In the previous studies, because $\mathrm{PV}_{y}=\beta+F u_{0}$ for a uniform westerly wind $u_{0}, C_{g}, \lambda, \delta$, and $\Delta \omega$ are only related to the strength of the uniform background westerly wind $u_{0}$. But in the extended NMI model, $C_{g}, \lambda, \delta$, and $\Delta \omega$ are not only related to the strength of the background westerly wind $U(y)$, but also related to $U_{y y}$ because of $\mathrm{PV}_{y}=$ $\beta-U_{y y}+F U$. Naturally, it is concluded that the change of the background westerly wind in its strength and meridional distribution can probably influence eddy-driven dipole blocking through changing $\mathrm{PV}_{y}$. Thus, the ENMI model here is a generalization of our previous NMI model derived by Luo and Li (2000), Luo (2005), and Luo et al. (2014) for $\mathrm{PV}_{y}=\beta+F u_{0}$.

In Eq. (2c), the phase speed of the blocking carrier wave is $C_{p}=\omega / k$, which represents the movement speed of the blocking only in a linear framework that requires the blocking amplitude to be small. However, the movement speed of a large-amplitude blocking in a nonlinear framework depends not only on the value of $C_{p}$, but also on the magnitude of the blocking amplitude. According to Luo (2000), the soliton solution of Eq. (2h) without eddy forcing term is $B=M_{0}$ sech $\left[\sqrt{\delta / 2 \lambda} M_{0}\left(x-C_{g} t\right)\right] \exp \left(-i \delta M_{0}^{2} t / 2\right)$, where $M_{0}=|B|_{\max }$ denotes the maximum amplitude. It is also easy to obtain the nonlinear phase speed of the blocking dipole as $\quad C_{\mathrm{NP}}=C_{p}+C_{N}=U-\mathrm{PV}_{y} /\left(k^{2}+m^{2}+F\right)-\delta_{N} M_{0}^{2} /$ $\left(2 k \mathrm{PV}_{y}\right)$ [where $C_{N}=-\delta_{N} M_{0}^{2} /\left(2 k \mathrm{PV}_{y}\right)$ is the blockinginduced phase speed and represents a westward speed if $\left.C_{N}<0\right]$. This result also holds and can be obtained when 
the eddy forcing is considered as a small perturbation of blocking. The same nonlinear phase speed formula can also be derived for a nonzonal basic flow $(U, V)$ that satisfies $\mathrm{PV}_{y}=\bar{\zeta}_{y}+\beta+F U$, where $\bar{\zeta}_{y}=\partial \bar{\zeta} / \partial y$ and $\bar{\zeta}=\partial V / \partial x-\partial U / \partial y$ is the relative vorticity of the nonzonal background wind. In this nonlinear phase speed formula, $M_{0}$ and $C_{g}$ are time dependent as the blocking intensifies and decays because of the eddy forcing (Luo 2000). The above nonlinear phase speed formula considers the effect of blocking amplitude on the blocking movement. Thus, the zonal movement of an observed blocking can be reflected by the magnitude of the nonlinear phase speed $C_{\mathrm{NP}}$.

It is supposed that Eq. (2h) has a soliton solution. Thus, the blocking wavy anomaly also has the soliton solution of $\psi_{B}=\sqrt{2 / L_{y}} M_{0} \operatorname{sech}\left[\sqrt{\delta /(2 \lambda)} M_{0}\left(x-C_{g} t\right)\right] \exp [i k(x-$ $\left.\left.C_{\mathrm{NP}} t\right)\right] \sin (m y)+\mathrm{cc}$ if the group velocity $C_{g}$ of the blocking wave packet $|B|$ is not changed with the change of the blocking amplitude $M_{0}$. In this case, the nonlinear energy dispersion of the blocking wavy anomaly can be characterized by $C_{\mathrm{ND}}=C_{g}-C_{\mathrm{NP}}=C_{g p}$ $C_{N}=2 k^{2} \mathrm{PV}_{y} /\left[\left(k^{2}+m^{2}+F\right)^{2}\right]+\delta_{N} M_{0}^{2} /\left(2 k \mathrm{PV}_{y}\right)$, where $C_{g p}=C_{g}-C_{p}$ is the linear energy dispersion and $C_{g p}=$ $2 k^{2} \mathrm{PV}_{y} /\left[\left(k^{2}+m^{2}+F\right)^{2}\right]$. This means that the nonlinear energy dispersion of observed blocking depends not only on the magnitude of background meridional PV gradient, but also on the blocking amplitude. When $M_{0} \sim 0$, the nonlinear energy dispersion $C_{\mathrm{ND}}$ reduces to the linear energy dispersion $C_{g p}$. Thus, the nonlinear energy dispersion of small-amplitude blocking can be approximately characterized by the linear energy dispersion $C_{g p}$. The lifetime or duration of observed blocking can be better reflected by the magnitude of the nonlinear energy dispersion $C_{\mathrm{ND}}$. The nonlinear energy dispersion of the blocking is enhanced when the blocking amplitude becomes large. In other words, larger amplitude blocking can more rapidly disperse its energy, which has been confirmed by the results of Luo (2000) and Yao et al. (2017), who found that a very largeamplitude blocking has strong energy dispersion to produce a short-lived blocking. Moreover, we see that the first term of the nonlinear energy dispersion $C_{\mathrm{ND}}$ is proportional to $\mathrm{PV}_{y}$ and its second term is proportional to $1 / \mathrm{PV}_{y}$. It is also seen from the mathematical expression of $C_{\mathrm{ND}}$ that the extent of the effect of $1 / \mathrm{PV}_{y}$ depends on the blocking amplitude $M_{0}$. But $M_{0}^{2} / \mathrm{PV}_{y}$ becomes very large once when the blocking amplitude $M_{0}$ is very large. In other words, for a fixed $\mathrm{PV}_{y}$, the blocking is more easily maintained for a small-amplitude blocking case than for a large-amplitude case. This explains why the North Pacific blocking often has larger amplitude but shorter lifetime than the North Atlantic blocking (Diao et al. 2006).

It is also easy to obtain $\partial C_{\mathrm{ND}} / \partial \mathrm{PV}_{y}=2 k^{2} /\left[\left(k^{2}+\right.\right.$ $\left.\left.m^{2}+F\right)^{2}\right]-\delta_{N} M_{0}^{2} /\left[2 k\left(\mathrm{PV}_{y}\right)^{2}\right]$. From this expression, one can obtain a critical value of $\mathrm{PV}_{y}$ as $\left(\mathrm{PV}_{y}\right)_{C}=$ $\sqrt{\delta_{N} / k}\left[M_{0}\left(k^{2}+m^{2}+F\right) /(2 k)\right]$. Thus, when $\mathrm{PV}_{y}<\left(\mathrm{PV}_{y}\right)_{C}$ for $\partial C_{\mathrm{ND}} / \partial \mathrm{PV} V_{y}<0$, the nonlinear energy dispersion $C_{\mathrm{ND}}$ increases with the decreased $\mathrm{PV}_{y}$. But when $\mathrm{PV}_{y}>\left(\mathrm{PV}_{y}\right)_{C}$ for $\partial C_{\mathrm{ND}} / \partial \mathrm{PV}_{y}>0$, the nonlinear energy dispersion $C_{\mathrm{ND}}$ decreases with the decreased $\mathrm{PV}_{y}$. The above results reveal that while the reduced strength of $\mathrm{PV}_{y}$ favors the weakened energy dispersion of blocking, it must be larger than a critical value $\left(\mathrm{PV}_{y}\right)_{C}$. When the blocking amplitude $M_{0}$ is larger, $\mathrm{PV}_{y}>\left(\mathrm{PV}_{y}\right)_{C}$ is not easily satisfied because of $\left(\mathrm{PV}_{y}\right)_{C}$ being larger, but $\mathrm{PV}_{y}<\left(\mathrm{PV}_{y}\right)_{C}$ is easily satisfied. This means that the eddy-driven dipole blocking with a longer lifetimes or weaker energy dispersion must require that the blocking amplitude cannot be too large or the background $\mathrm{PV}_{y}$ cannot be too small. Of course, this result is obtained based on an important assumption that the group velocity of the blocking is not changed with the blocking evolution.

It is further found that the nonlinear phase speed $C_{\mathrm{NP}}$ of the eddy-driven dipole blocking depends not only on the magnitudes of $U$ and $\mathrm{PV}_{y}$, but also on the blocking amplitude $M_{0}$. When $\mathrm{PV}_{y}>0$, the amplification of the blocking amplitude can induce a westward movement of blocking. The blocking amplitude-induced westward movement speed may be understood as the effect of weakened mean zonal wind induced by the amplified blocking amplitude $|B|$. This can also be explained in terms of the mean westerly wind anomaly change within the blocking region. In fact, the westward speed $C_{N}=-\delta_{N} M_{0}^{2} /\left(2 k \mathrm{PV}_{y}\right)$ is proportional to $-|B|^{2}$ and equivalent to the effect of weakened mean zonal wind within the blocking region described by $U_{m}=$ $-\partial \psi_{m} / \partial y=-|B|^{2} \sum_{n=1}^{\infty} q_{n} g_{n}(n+1 / 2) m \sin (n+1 / 2) m y \mathrm{du}-$ ring the blocking episode. As noted below, $U_{m}$ is weakened within the blocking region because of the effect of $-|B|^{2}$ as the blocking intensifies. In this case, the blocking moves westward. Also, there is $C_{\mathrm{NP}} \approx C_{p}$ when the blocking amplitude is smaller $\left(M_{0} \sim 0\right)$. This case corresponds to a linear blocking theory as noted above. This linear theory has some limitations because it cannot explain why the intensified blocking dipole shows a strong westward movement (Fig. 2a). We further find that the meridional structure of the dipole blocking is dominated by the meridional distributions of the linear dispersion $\lambda$ and nonlinearity $\delta$ terms, even though the amplitude $M_{0}$ and group velocity $C_{g}$ of the blocking wave packet are varied with time under the eddy forcing (Luo 2000). 
While $C_{g}$ represents the propagation speed of the blocking wave packet, the behavior (movement, strength, and duration) of eddy-driven dipole blocking is mainly influenced by the background parameters $\lambda, \delta$, and $\Delta \omega$ associated with the background westerly wind distribution. It is also noted that in Eq. (2h) the linear dispersion term $\lambda$ is proportional to $\mathrm{PV}_{y}$ and the nonlinearity strength $\delta$ is proportional to $1 / \mathrm{PV}_{y}$. The blocking system satisfies a linear energy dispersionnonlinearity strength inverse relation rule and has weak dispersion and strong nonlinearity when $\mathrm{PV}_{y}$ is small especially in high latitudes. Thus, the small $\mathrm{PV}_{y}$ is conducive to strong and long-lived dipole blocking for a given preexisting eddy strength. We can further obtain $-\Delta \omega=\mathrm{PV}_{y}\left[\tilde{k}_{2} /\left(\tilde{k}_{2}^{2}+m^{2} / 4+F\right)-\tilde{k}_{1} /\left(\tilde{k}_{1}^{2}+m^{2} / 4+\right.\right.$ $\left.F)-k /\left(k^{2}+m^{2}+F\right)\right]$ for $k=\tilde{k}_{2}-\tilde{k}_{1}$. Obviously, the duration of the eddy forcing term in Eq. (2h) is mainly determined by the magnitude of $\mathrm{PV}_{y}$ for fixed zonal wavenumbers $k$ and $\tilde{k}_{j}(j=1,2)$. The preexisting eddy forcing term has a long duration when $\mathrm{PV}_{y}$ is small. In our ENMI model, because $\lambda \propto \mathrm{PV}_{y}, \delta \propto 1 / \mathrm{PV}_{y}$, and $-\Delta \omega \propto \mathrm{PV}_{y}$, the strength and duration of eddy-driven dipole blocking are mainly related to the magnitude of $\mathrm{PV}_{y}$, whereas the movement of dipole blocking is related to $U$, $\mathrm{PV}_{y}$, and the blocking amplitude as noted above.

The above analysis leads us to conclude that an intense and long-lived dipole blocking is easily formed when $\mathrm{PV}_{y}$ is small, because $\lambda$ and $\Delta \omega$ are small and $\delta$ is large. The eddy-driven blocking dipole will have a strong meridional asymmetry in strength, persistence, movement, and meridional structure because of strong meridional asymmetries in the energy dispersion, nonlinearity, and the duration of the preexisting eddy forcing if $U$ or $\mathrm{PV}_{y}$ has a strong asymmetry in the meridional direction. This problem will be further investigated to explain why the North Atlantic blocking has the observed spatial structure and westward movement as shown in Fig. 2a.

The evolutions of planetary- and synoptic-scale streamfunction fields and their total streamfunction field during the blocking life cycle can be obtained once the solution of the blocking amplitude $B$ is obtained. Here, a high-order split-step Fourier scheme (Muslu and Erbay 2005) is used to solve Eq. (2h) to obtain the spatiotemporal solution of the blocking amplitude $B$ for prescribed initial values and parameter conditions. Here, the periodic boundaries with $L_{x}= \pm 11.48$ are used to compute Eq. (2h). The assumption of $f_{0}(x)=$ $a_{0} \exp \left[-\mu \varepsilon^{2}\left(x+x_{T}\right)^{2}\right]$ used to represent the slowly varying amplitude distribution of preexisting synoptic-scale eddies in this paper does not destroy the periodic boundary condition of the calculation. In this paper, we only plot the streamfunction fields in the zonal region from $x=-5.74$ to $x=5.74$.
TABLE 1. Values of given parameters used to calculate the ENMI model solution.

\begin{tabular}{lc}
\hline \hline \multicolumn{1}{c}{ Parameters } & Value \\
\hline $\begin{array}{l}\text { Nondimensional zonal wavenumber } k \\
\text { of the blocking dipole }\end{array}$ & $k=2 /\left(6.371 \cos \varphi_{0}\right)$ \\
$\begin{array}{l}\text { Nondimensional zonal wavenumber } \tilde{k}_{1} \\
\quad \text { of preexisting synoptic-scale eddies }\end{array}$ & $\tilde{k}_{1}=9 /\left(6.371 \cos \varphi_{0}\right)$ \\
Nondimensional zonal wavenumber $\tilde{k}_{2}$ & $\tilde{k}_{2}=11 /\left(6.371 \cos \varphi_{0}\right)$ \\
$\quad$ of preexisting synoptic-scale eddies & $55^{\circ} \mathrm{N}$ \\
Reference latitude $\varphi_{0}$ & 5 \\
Nondimensional width $L_{y}$ of the $\beta$ & $10^{6} \mathrm{~m}$ \\
$\quad$ channel & $10 \mathrm{~m} \mathrm{~s}{ }^{-1}$ \\
Characteristic horizontal length $\tilde{L}$ & 1.2 \\
Characteristic horizontal wind speed $\tilde{U}$ & 0.24 \\
$\mu \quad$ & $2.87 / 2$ \\
$\varepsilon \quad$ Zonal location $x_{T}$ of preexisting synoptic- & \\
$\quad$ scale eddies & \\
Amplitude $a_{0}$ of preexisting synoptic-scale & \\
$\quad$ eddies &
\end{tabular}

\section{Role of $P V_{y}$ in determining the lifetime and intensity of a blocking event}

\section{a. Effect of different $P V_{y}$ with constant background zonal wind}

In this section, we first examine the effect of constant $\mathrm{PV}_{\mathrm{y}}$ on the blocking dipole. For the parameters shown in Table 1, we consider two cases: 1) $U=u_{0}=0.7$ and $\mathrm{PV}_{y} \approx 2.0$ and 2) $U=0.7$ and $\mathrm{PV}_{y} \approx 2.5$. Note that all values are in units of their characteristic scales as all variables are nondimensional (i.e., normalized by the characteristic scales). The choice of $U=0.7$ can allow a given initial blocking dipole to be stationary. Here $\mathrm{PV}_{y} \approx 2.0$ is the value of $\mathrm{PV}_{y}$ at $55^{\circ} \mathrm{N}$ for $U=0.7$, but $\mathrm{PV}_{y} \approx 2.5$ at $38^{\circ} \mathrm{N}$ for $U=0.7$. To clearly see the impact of the $\mathrm{PV}$ gradient, we fix $U$, but change $\mathrm{PV}_{y}$.

For the initial amplitude $B(x, 0)=0.4$, background westerly wind $U=0.7$, and other parameters listed in Table 1, we show the time sequences of instantaneous planetary-scale streamfunction $\psi_{P}$, blocking wavy anomaly $\psi_{B}$, synoptic-scale streamfunction $\psi^{\prime}$, and total streamfunction $\psi_{T}$ fields during the life cycle of an eddy-driven dipole blocking event in Figs. 3 and 4 for $\mathrm{PV}_{y}=2.0$ (Fig. 3) and $\mathrm{PV}_{y}=2.5$ (Fig. 4) cases. As seen in the $\psi_{P}$ and $\psi_{B}$ fields in Fig. 3a, the prespecified initial blocking can be amplified into a typical dipole blocking, which has a lifetime of 10-20 days under the dipole eddy forcing $-\nabla \cdot\left(\mathbf{v}_{1}^{\prime} q_{1}^{\prime}\right)_{P}$ induced by preexisting synoptic-scale eddies upstream. During the mature phase (from days 6 to 9), the blocking dipole in the planetary-scale field $\psi_{P}$ (Fig. 3a) resembles the modon solution of the blocking (McWilliams 1980), while the modon solution cannot reflect the life cycle 

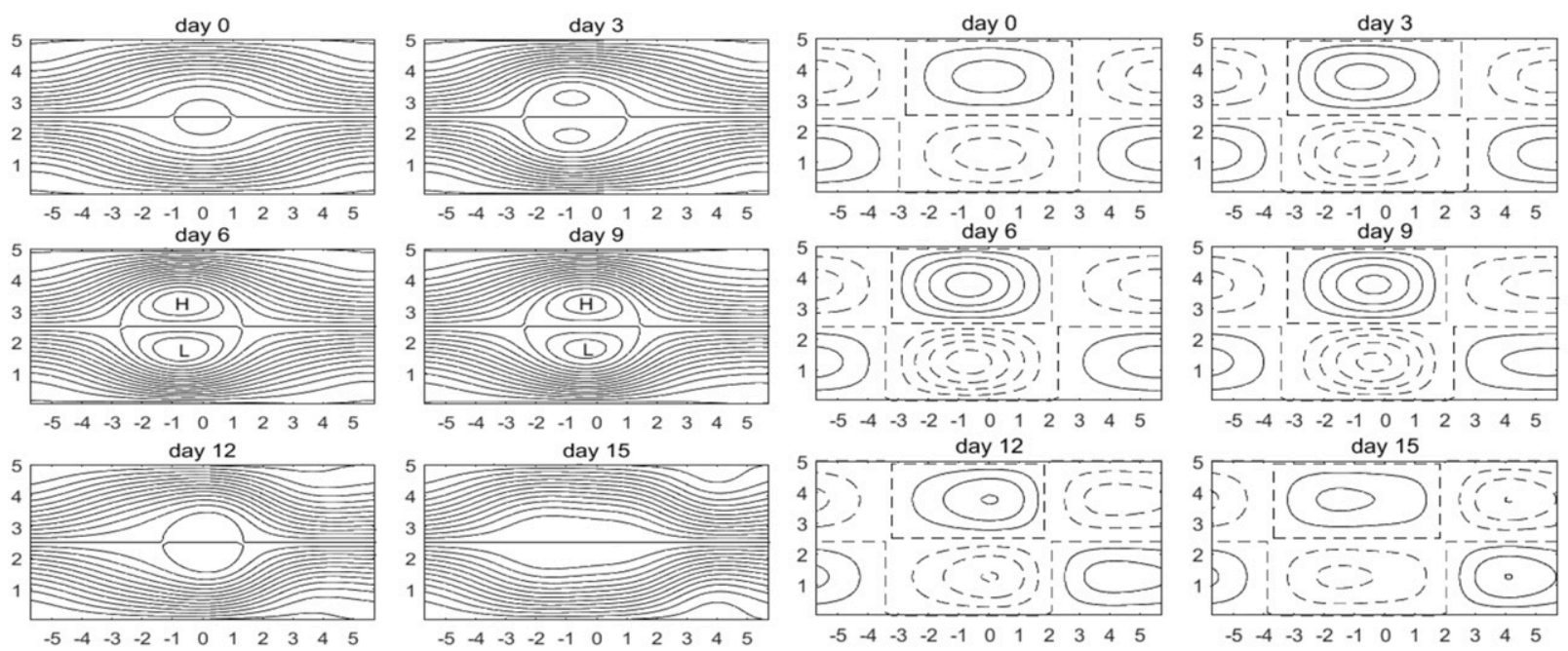

(a) $\psi_{P}$

(b) $\psi_{B}$
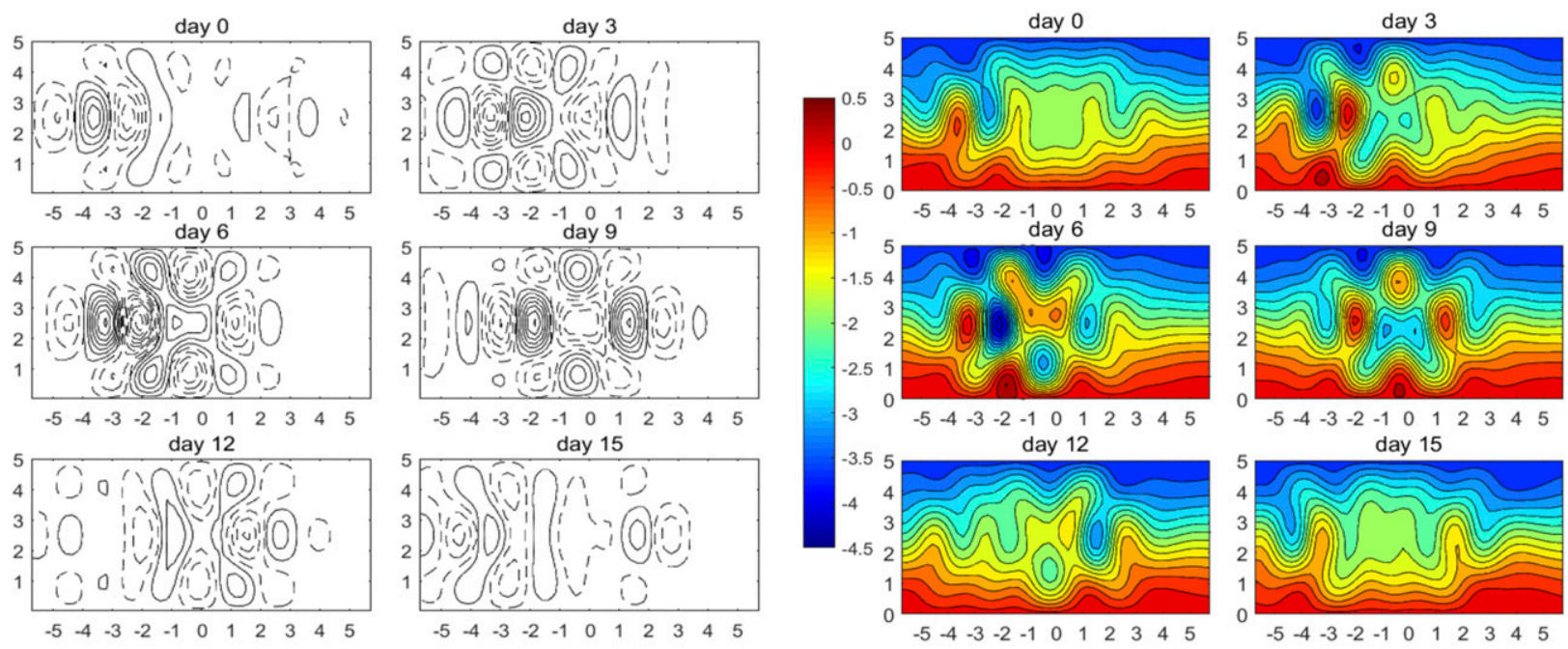

(c) $\psi^{\prime}$

(d) $\psi_{T}$

FIG. 3. Instantaneous fields of (a) planetary-scale streamfunction $\psi_{P}(\mathrm{CI}=0.15)$, (b) blocking wavy anomaly $\psi_{B}(\mathrm{CI}=0.2)$, (c) synopticscale streamfunction $\psi^{\prime}(\mathrm{CI}=0.3)$, and $(\mathrm{d})$ total streamfunction $\psi_{T}(\mathrm{CI}=0.3)$ fields during the life cycle of an eddy-driven dipole blocking for $\mathrm{PV}_{y}=2.0$ with the initial amplitude of $B(x, 0)=0.4$ and $U=0.7$; other parameters are listed in Table 1 . The nondimensional $y(x)$ coordinate is marked by the ordinate (abscissa).

of observed dipole blocking and associated westerly jet meandering (Fig. 1).

The total streamfunction field of the dipole blocking also shows a large meandering of midlatitude westerly jet streams (the $\psi_{T}$ fields in Figs. 3d and $4 \mathrm{~d}$ ) because of eddy deformation (the $\psi^{\prime}$ fields in Figs. 3c and 4c), first observed by Berggren et al. (1949) and then by Luo (2000) from a theoretical model. In other words, the meandering westerly jet stream reflects the synoptic time-scale evolution of a blocking flow and the northward (southward) intrusion of associated warm subtropical (cold Arctic) air (Luo 2000, 2005; Pelly and Hoskins 2003; Luo et al. 2014). We also note that the basic features of the modeled blocking are not sensitive to the initial conditions of the blocking (not shown). A comparison between Figs. 3 and 4 indicates that the meandering westerly jet stream is more persistent or stationary for a small PV gradient $\left(\mathrm{PV}_{y}=2.0\right.$; Fig. 3d $)$ than for a large $\mathrm{PV}$ gradient $\left(\mathrm{PV}_{y}=2.5\right.$; Fig. $\left.4 \mathrm{~d}\right)$ because the blocking dipole moves westward slower (see the $\psi_{B}$ panels in Figs. $3 \mathrm{~b}$ and $4 \mathrm{~b}$ ) and has a longer lifetime (see the $\psi_{P}$ 

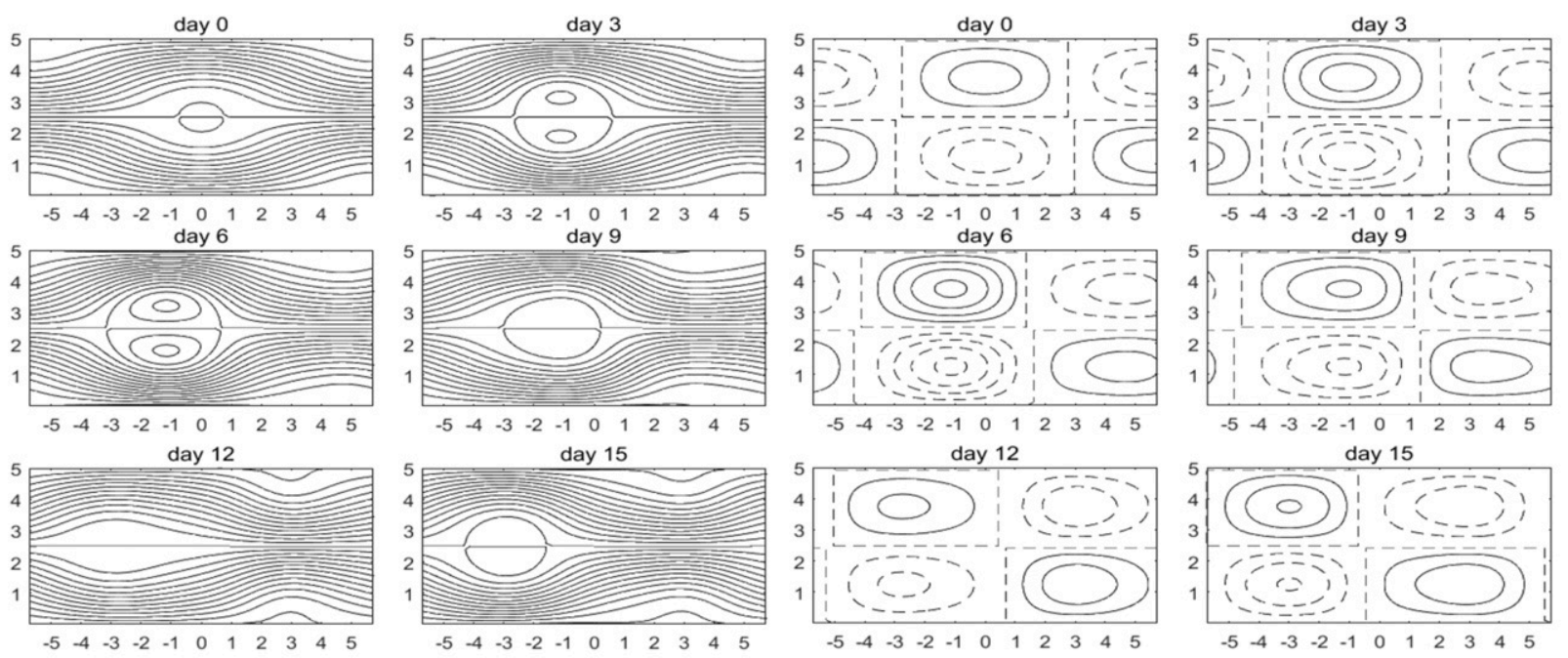

(a) $\psi_{P}$

(b) $\psi_{B}$
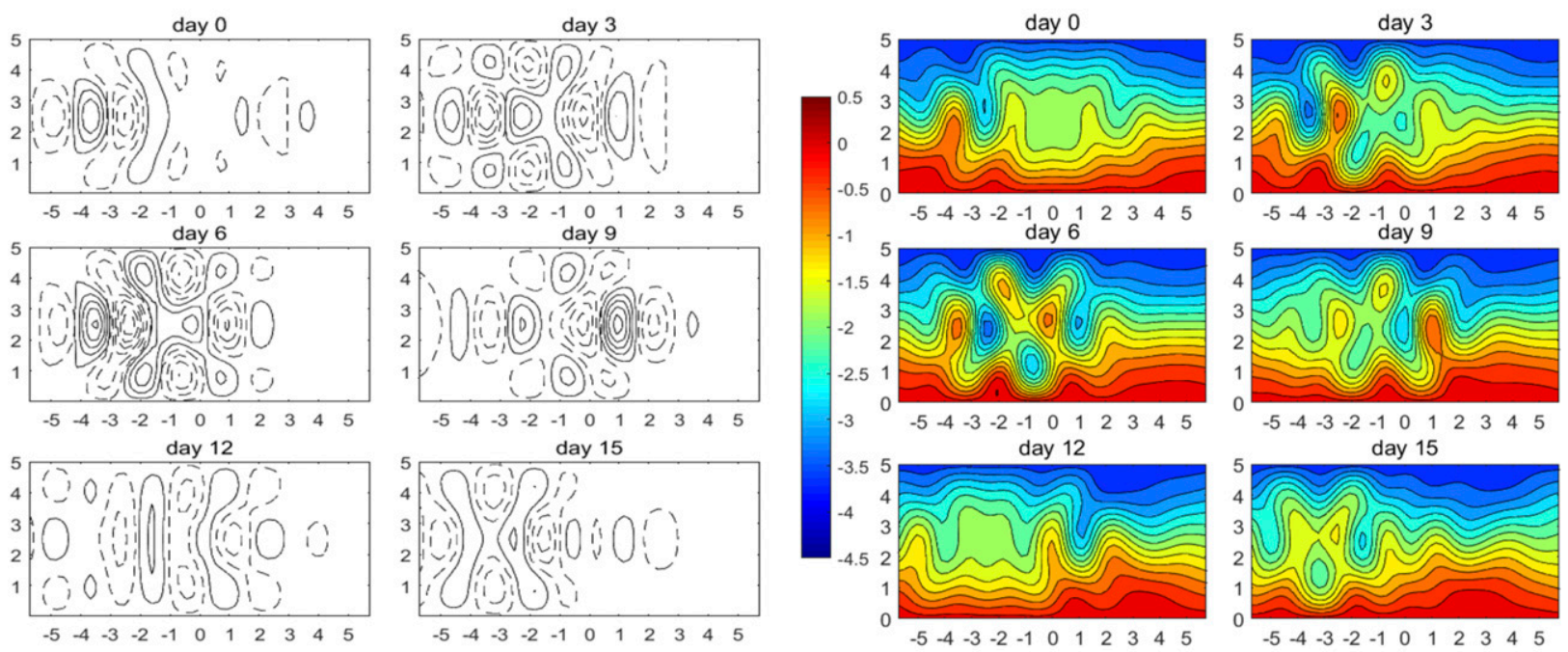

(c) $\psi^{\prime}$

(d) $\psi_{T}$

FIG. 4. As in Fig. 3, but for $\mathrm{PV}_{y}=2.5$.

panels in Figs. $3 \mathrm{a}$ and $4 \mathrm{a}$ ) for $\mathrm{PV}_{y}=2.0$ than for $\mathrm{PV}_{y}=$ 2.5. This implies that as a background condition the reduced PV gradient can lengthen the lifetimes of dipole blocking and associated meandering westerly jet stream.

To examine the sensitivity of our results to the initial amplitude of the dipole blocking and to quantify the changes in amplitude and duration of the intensified blocking event, here we further examine a case with $B(x, 0)=0.45$. We also define $\psi_{D}=\left(\psi_{B}\right)_{\text {Max }}-\left(\psi_{B}\right)_{\text {Min }}$ as the daily amplitude of dipole blocking during its life cycle, where $\left(\psi_{B}\right)_{\text {Max }}$ and $\left(\psi_{B}\right)_{\text {Min }}$ denote the maximum and minimum values of the streamfunction anomaly within the blocking region of the blocking dipole, respectively. For the same condition as in Fig. 3, we show the instantaneous fields of planetary-scale streamfunction $\psi_{P}$ of an eddy-driven dipole blocking event in Figs. 5a and 5b for $\mathrm{PV}_{y}=2.0$ and $\mathrm{PV}_{y}=2.5$. It is seen that the eddy-driven dipole blocking has a shorter lifetime for a larger $\mathrm{PV}_{y}$ (Fig. 5b) than that for a smaller $\mathrm{PV}_{\mathrm{y}}$ (Fig. 5a), even when the initial blocking amplitude is large. The temporal variations of the daily amplitude $\psi_{D}$ of the dipole blocking are shown in Fig. 5c (Fig. 5d) for $B(x, 0)=0.4[B(x, 0)=$ $0.45]$ with $\mathrm{PV}_{y}=2.0$ (solid line) and $\mathrm{PV}_{y}=2.5$ (dashed line). It is clearly seen that the eddy-driven dipole 

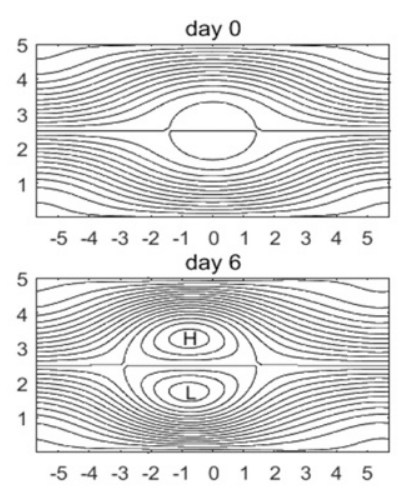

day 12

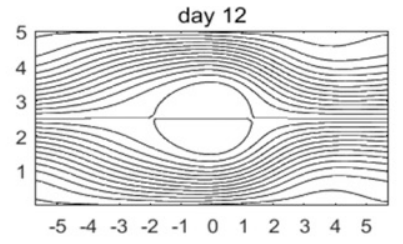

(a)

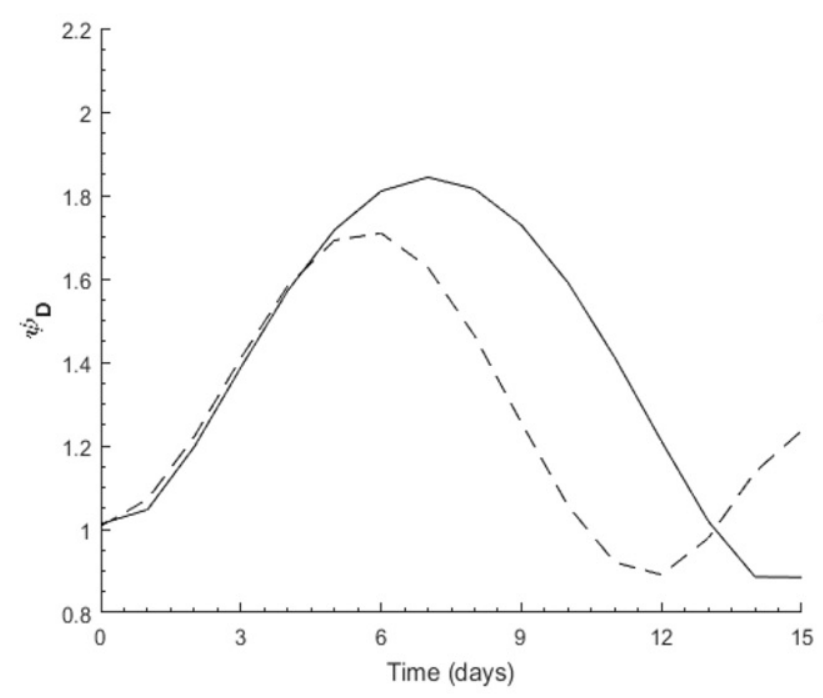

(c)
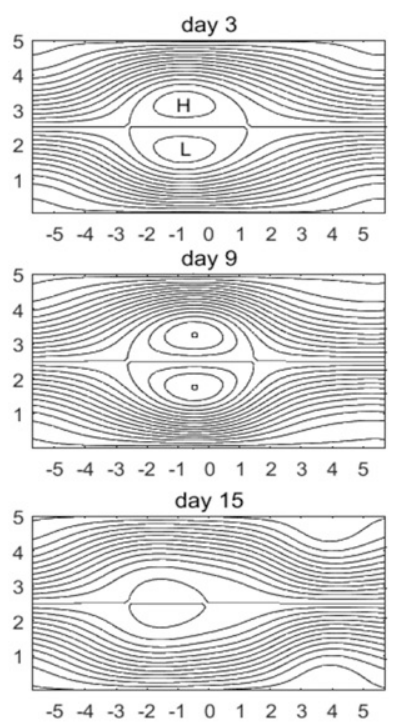
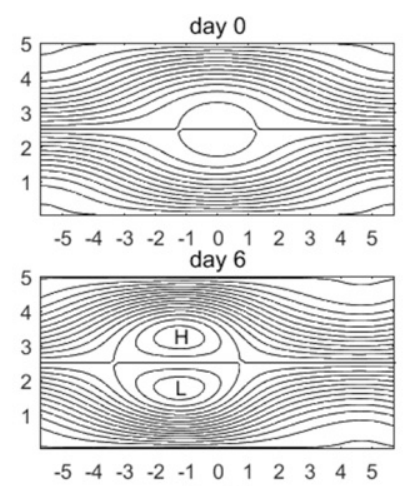

day 12

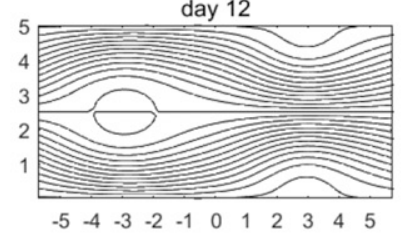

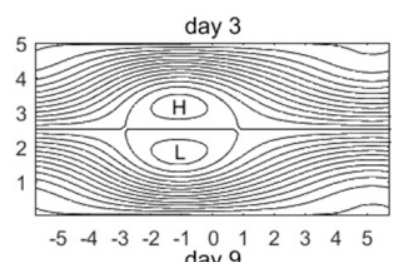

day 9

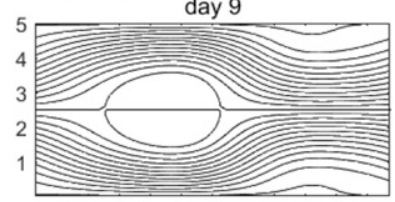

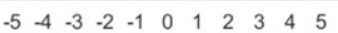
day 15

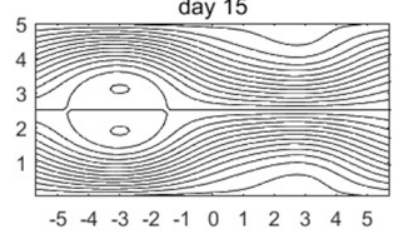

(b)

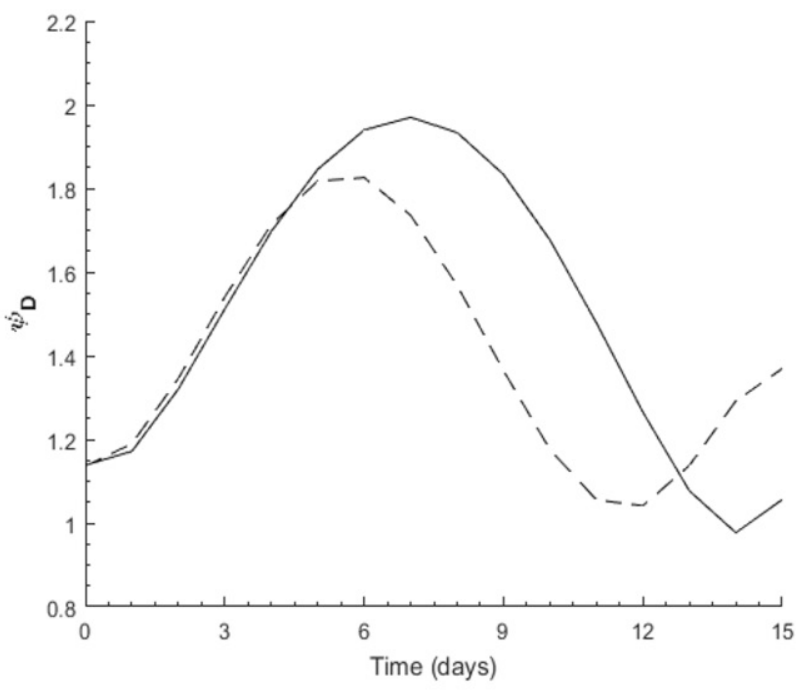

(d)

FIG. 5. (top) Temporal evolution of instantaneous planetary-scale streamfunction $\psi_{P}$ fields $(\mathrm{CI}=0.15)$ of a dipole blocking event for (a) $\mathrm{PV}_{y}=2.0$ and (b) $\mathrm{PV}_{y}=2.5$ with the initial amplitude of $B(x, 0)=0.45$ and the same parameters as in Fig. 3. (bottom) Temporal variations of the daily blocking dipole amplitude $\psi_{D}=\left(\psi_{B}\right)_{\operatorname{Max}}-\left(\psi_{B}\right)_{\operatorname{Min}}$ for (c) $B(x, 0)=0.4$ and (d) $B(x, 0)=0.45$ with PV $y=2.0$ (solid line) and $\mathrm{PV}_{y}=2.5$ (dashed line), where $\left(\psi_{B}\right)_{\mathrm{Max}}$ and $\left(\psi_{B}\right)_{\text {Min }}$ denotes the maximum and minimum values of the blocking dipole within the blocking region, respectively.

blocking has a larger amplitude and a longer lifetime in an environment with a small PV gradient than in an environment with a large PV gradient for both $B(x, 0)=0.4$ and $B(x, 0)=0.45$. The above results suggest that a small meridional PV gradient is a favorable environment for strong and long-lived dipole blocking. Further calculations showed that within the blocking region the kinetic energy is nearly conserved and the enstrophy increases as the blocking grows (not shown). This implies that the potential vorticity gradient affects the blocking may be through a cascade of enstrophy from waves with large wavenumbers (small scales) to waves with small wavenumbers (large scales). 


\section{b. Causal linkage between the background PV gradient and the lifetime of blocking}

It is easy to find from Eq. (2) that the mean zonal wind anomaly $U_{m}=-\partial \psi_{m} / \partial y$ changes with time as a response to the blocking evolution because of the inclusion of the blocking amplitude $B$. This can cause the PV to vary during the life cycle of blocking, which represents a feedback of the blocking on the PV gradient. The time-dependent zonally averaged $\mathrm{PV}$ gradient can be obtained as $\left(\mathrm{PV}_{y}\right)_{\text {life }}=\mathrm{PV}_{y}+$ $\mathrm{PV}_{y}\left(U_{m}\right)$ during the blocking life cycle from Eq. (2), where $\mathrm{PV}_{y}$ is the background $\mathrm{PV}$ gradient and $\mathrm{PV}_{y}\left(U_{m}\right)=-\left(U_{m}\right)_{y y}+F U_{m}\left[\left(U_{m}\right)_{y y}=\partial^{2} U_{m} / \partial y^{2}\right]$ is the time-dependent PV gradient anomaly due to the mean zonal wind change $U_{m}$. As shown in Figs. 3 and 4 , the dipole blocking is strong and long lived as the background $\mathrm{PV}_{y}$ prior to the blocking onset is small (Fig. 3a). Accompanied by the intensifying of blocking, the mean zonal wind $U_{m}$ is weakened within the blocking region (Figs. 6a,b) because of the effect of the blocking amplitude $|B|^{2}$, which favors the westward movement of blocking. We see that the mean zonal wind is split into two branches around the blocking region and the presence of long-lived blocking causes a larger weakening of the mean zonal wind within the blocking region (Fig. 6a) than the short-lived one (Fig. 6b). It also corresponds to a smaller PV gradient within the blocking region (Fig. 6c) because of stronger weakening of mean zonal winds than for the short-lived case (Fig. 6d). We show the time variations of domain-averaged $\mathrm{PV}_{y}\left(U_{m}\right)\left(\right.$ Fig. 7a) and $\left(\mathrm{PV}_{y}\right)_{\text {life }}$ (Fig. 7b) over $-3.0 \leq$ $x \leq 2.0$ and $2.0 \leq y \leq 3.0$ during the blocking life cycle in for $\mathrm{PV}_{y}=2.0$ and $\mathrm{PV}_{y}=2.5$. It is further found that the $\mathrm{PV}_{y}\left(U_{m}\right)$ anomaly decreases with the growth of blocking, and the decrease is larger for the longlived blocking (solid line in Fig. 7a) than for the shortlived blocking (dashed line in Fig. 7a). As a result, the total PV gradient $\left(\mathrm{PV}_{y}\right)_{\text {life }}$ is smaller during the blocking episode for a long-lived blocking (solid line in Fig. 7b) than for a short-lived blocking (dashed line in Fig. 7b), which is also smaller during the blocking mature period than during the prior or latter period of the blocking. This indicates that the long-lived blocking requires a smaller prior background PV gradient than the short-lived blocking. These results suggest the following causal relationship between the PV gradient and blocking: a small background PV gradient $\rightarrow$ long-lived blocking dipole $\rightarrow$ strong reduction and splitting of mean zonal wind $\rightarrow$ a smaller PV gradient within the blocking region. In other words, the small background PV gradient is a prerequisite of long-lived blocking, which further reduces the PV gradient within the blocking region.

\section{c. Effect of $P V_{y}$ associated with weakened background zonal wind}

While some studies have noted that the double jets tend to favor atmospheric blocking (Ogi et al. 2004; Tachibana et al. 2010), how they favor blocking is not addressed in these previous studies. Here, we use the ENMI model to address this issue. To understand the impact of the spatial structure of the background zonal wind on atmospheric blocking in persistence, intensity, and spatial shape, we fist consider a symmetric double-jet structure in the form of $U=u_{0}+\Delta u \cos (m y)$, where $\Delta u$ is a constant. For $B(x, y, 0)=0.4$ and the same parameters as in Fig. 3a, the instantaneous planetary-scale streamfunction $\psi_{P}$, anomaly streamfunction $\psi_{B}$, synoptic-scale streamfunction $\psi^{\prime}$, and total streamfunction $\psi_{T}$ fields are shown in Fig. 8 for $u_{0}=0.7$ and $\Delta u=0.2$. Because $\lambda$ and $\delta$ are two more important parameters for determining the energy dispersion and nonlinearity of the dipole blocking, it is useful to show the variations of $U, \Delta \omega, \mathrm{PV}_{y}, C_{g}, \lambda$, and $\delta$ with the latitude and the time evolution of the blocking amplitude $\psi_{D}$ in Fig. 9. It is worth noting that in our ENMI model, the nonlinear behavior of blocking is mainly influenced by the parameters $\lambda$ and $\delta$ of the NLS equation [Eq. (2h)], whereas the duration of the blocking is determined by the value of $\Delta \omega$. When $\mathrm{PV}_{y} \rightarrow 0$ (PV homogenization), one can have $C_{p} \rightarrow U, C_{g} \rightarrow U, \lambda \rightarrow 0$, $\Delta \omega \rightarrow 0$, and $\delta \rightarrow \infty$. In this case, the dipole blocking can maintain itself for a long lifetime because of the small energy dispersion and strong nonlinearity, even though it propagates eastward with $U$, which may affect the duration observed at a fixed location. The lifetime discussed here is for the whole duration of the blocking system over a moving path, while the duration of the blocking at a fixed location (which is important for local weather) is also affected by its moving speed.

On the other hand, because the background zonal wind changes with the latitude, the synoptic-scale eddies (Fig. 8c) are further distorted by the varying background zonal winds in the meridional direction in addition to the blocking-induced eddy deformation (Luo et al. 2014). Such an effect is more evident during the blocking decay phase. While the total streamfunction field (Fig. 8d) shows an intensely meandering jet stream, it has different spatial structures after day 9, unlike those found in the $\psi_{T}$ field in Fig. 3d. It seems that the nonuniform meridional shear of background zonal winds can break up synoptic-scale anticyclones or cyclones, as suggested by Thorncroft et al. (1993). But such a wave breaking is not the cause of the blocking 


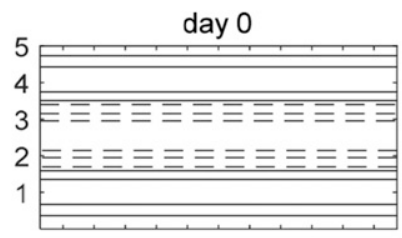

$-5-4-3-2-100123345$ day 6

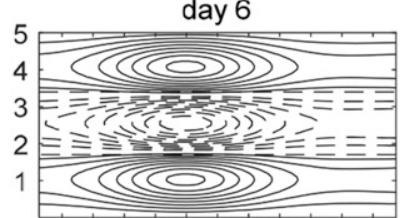

$-5-4-3-2-1 \quad 0 \quad 1 \quad 2 \quad 3445$ day 12

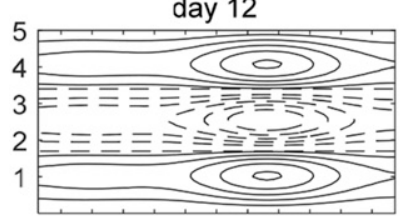

$-5-4-3-2-1 \quad 0 \quad 1223445$

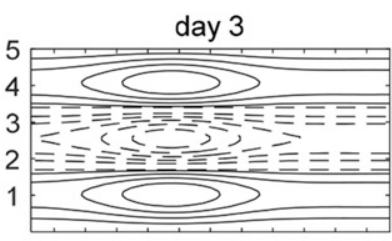

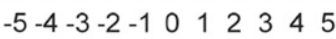

day 9

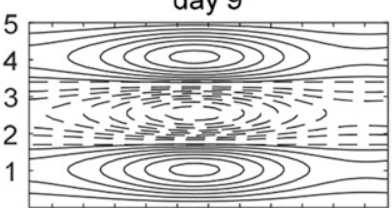

$-5-4-3-2-1 \quad 0 \quad 1 \quad 2 \quad 3445$

day 15

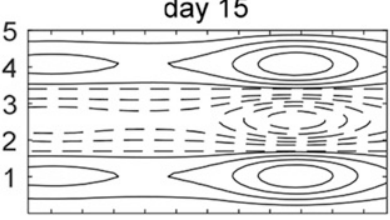

$-5-4-3-2-11001123345$

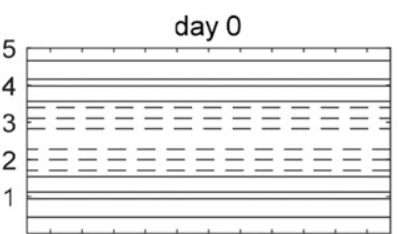

$\begin{array}{llllllllll}-5 & -4 & -3 & -2 & -1 & 0 & 1 & 2 & 3 & 4\end{array}$

day 6

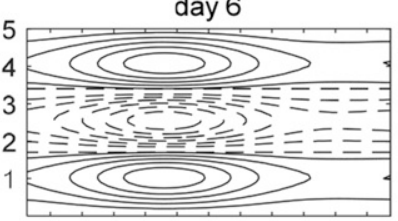

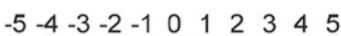

day 12

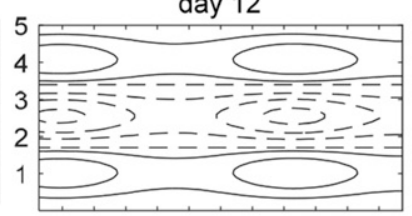

$\begin{array}{llllllllll}-5 & -4 & -3 & -2 & -1 & 0 & 1 & 2 & 3 & 4\end{array}$

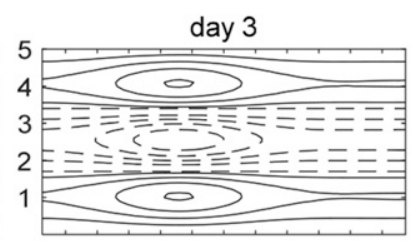

$\begin{array}{llllllllll}-5 & -4 & -3 & -2 & -1 & 0 & 1 & 2 & 3 & 4\end{array}$ day 9

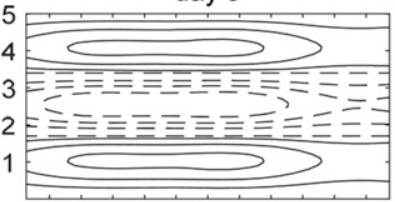

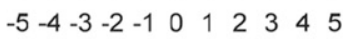
day 15

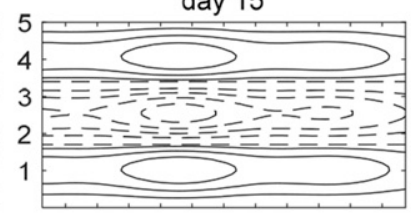

$-5-4-3-2-1 \quad 0 \quad 1 \quad 2 \quad 3445$

(a)

(b)
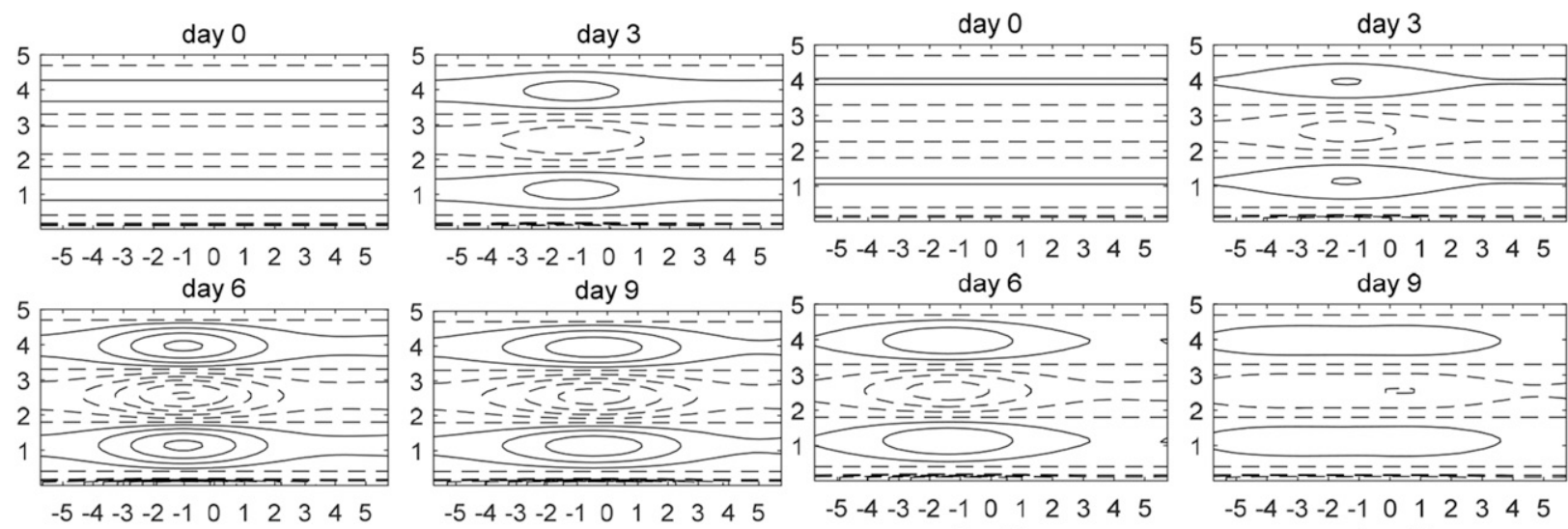
day 12
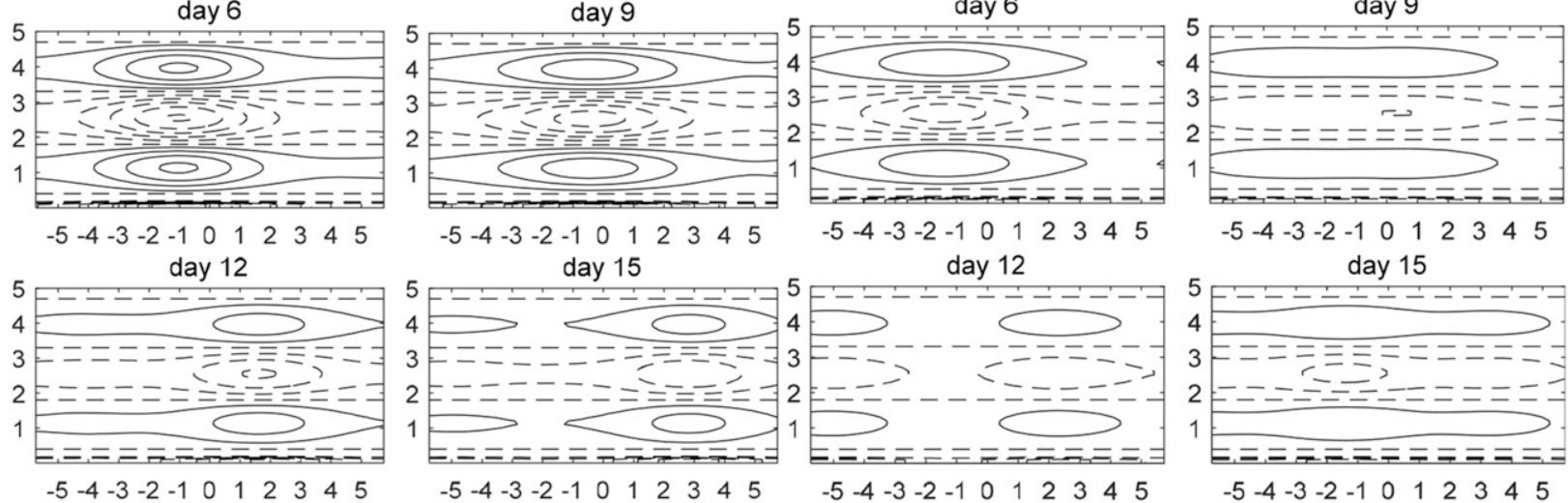

$\begin{array}{llllllllll}-5 & -4 & -3 & -2 & -1 & 0 & 1 & 2 & 3 & 4\end{array}$ day 15
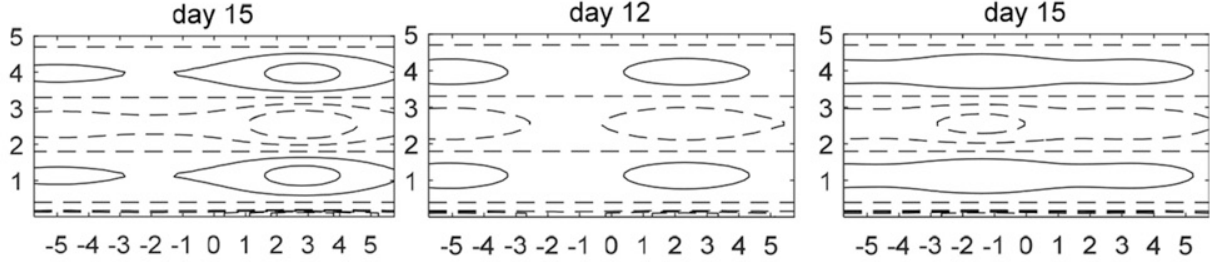

(d)

FIG. 6. Time sequences of (a),(b) mean zonal wind $U_{m}=-\partial \psi_{m} / \partial y(\mathrm{CI}=0.05)$ and (c),(d) PV gradient $\mathrm{PV}_{y}\left(U_{m}\right)=-\left(U_{m}\right)_{y y}+F U_{m}$ $(F=1, \mathrm{CI}=0.5)$ during the blocking life cycle due to the feedback of intensified blocking for (a), (c) $\mathrm{PV}_{y}=2.0$ and $(\mathrm{b}),(\mathrm{d}) \mathrm{PV}_{y}=2.5$ under $U=0.7$.

maintenance (Luo et al. 2014). It is noted that the meridional average value of $U$ is $\bar{U}=\left(1 / L_{y}\right) \int_{0}^{L_{y}}\left[u_{0}+\right.$ $\Delta u \cos (m y)] d y=u_{0}$ (Fig. 9a). Also, the meridional average values of $\Delta \omega, \mathrm{PV}_{y}, C_{g}, \lambda$, and $\delta$ are independent of $\Delta u$ (Figs. 9b-f), but dependent on $u_{0}$. Although the strength of the domain-averaged double jet (Fig. 9a) is the same as that of the uniform westerly wind in Fig. 3a, we find that the eddy-driven dipole blocking under such a background double jet becomes slightly more persistent (solid line in Fig. 9g) than that in the uniform 


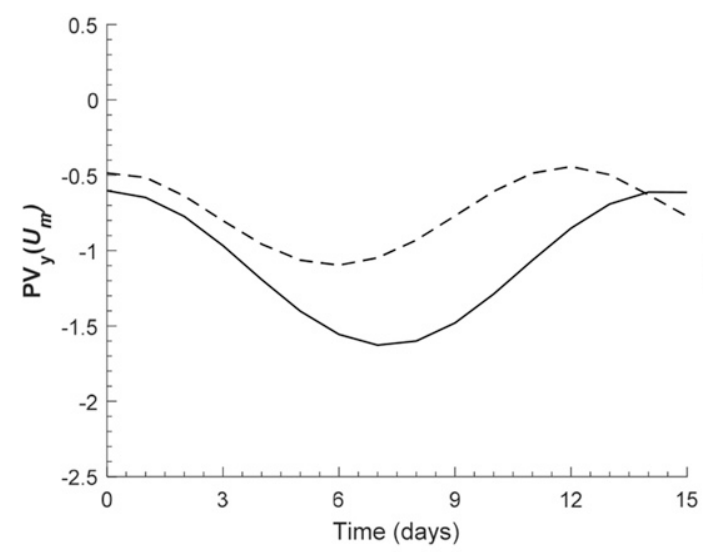

(a)

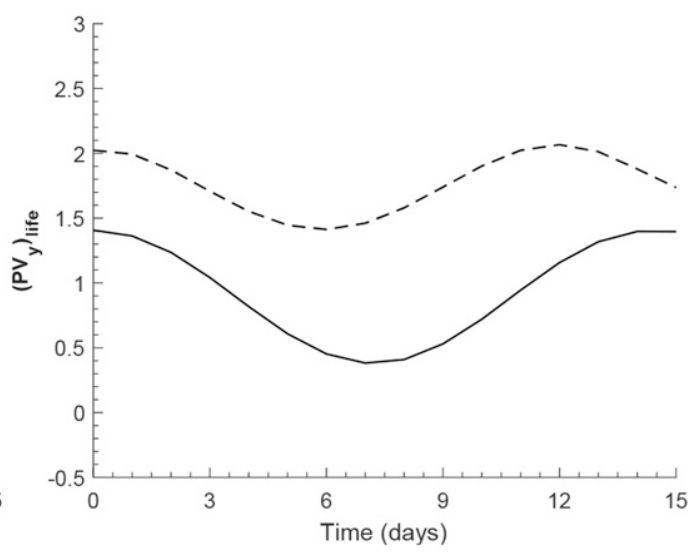

(b)

FIG. 7. Time series of the daily domain-averaged (a) $\mathrm{PV}_{y}\left(U_{m}\right)$ anomaly and (b) $\left(\mathrm{PV}_{y}\right)_{\text {life }}\left[\right.$ where $\left(\mathrm{PV}_{y}\right)_{\text {life }}=$ $\left.\mathrm{PV}_{y}+\mathrm{PV}_{y}\left(U_{m}\right)\right]$ averaged over $-3.0 \leq x \leq 2.0$ and $2.0 \leq y \leq 3.0$ during the blocking life cycle for $\mathrm{PV}_{y}=2.0$ (solid line) and $\mathrm{PV}_{y}=2.5$ (dashed line).

background westerly wind (dot-dashed line in Fig. 9g), while the blocking amplitude is almost independent of the value of $\Delta u$. Thus, although the meridionally averaged $\mathrm{PV}_{y}\left[\overline{\mathrm{PV}}_{y}=\left(1 / L_{y}\right) \int_{0}^{L_{y}} \mathrm{PV}_{y}\left(y^{\prime}\right) d y^{\prime}=\beta+F u_{0}\right]$ is the same as the uniform zonal wind case, the decreasing extent of the background zonal wind in the middle channel can somewhat increase the duration of the blocking.

It is seen from Fig. 9 that in the weakened region of the prescribed double jet $U, \lambda$ and $\Delta \omega$ are weakened (Figs. 9e and $9 \mathrm{~b}$ ) and $\delta$ is intensified (Fig. 9f) because of reduced $\mathrm{PV}_{y}$ (Fig. 9c) so that the dipole blocking has weak energy dispersion, strong nonlinearity, and long duration in the middle part of the blocking region. Such weakened energy dispersion, reduced $\Delta \omega$, and intensified nonlinearity distributions can increase the persistence of dipole blocking but cannot produce a meridional asymmetry. Thus, the meridional distribution of the background zonal wind prior to the blocking onset can significantly affect the dipole blocking in spatial shape and duration.

To show the sensitivity of the results to the value of $u_{0}$, Fig. 10b compares the temporal variations of the blocking amplitude $\psi_{D}$ for $\Delta u=0.2$, a case with $u_{0}=0.5$ and another with $u_{0}=0.9$ (and $\Delta u=0.2$ for both cases). It is seen that while the duration of the blocking depends slightly on the value of $\Delta u$ (Fig. 10a), the value of $u_{0}$ can strongly influence on the duration and amplitude of the blocking (Fig. 10b). Because the magnitude of the meridionally averaged $\mathrm{PV}_{y}\left(\overline{\mathrm{PV}}_{y}=\beta+F u_{0}\right)$ depends mainly on the strength of background zonal wind $u_{0}$, it appears that the change in the background zonal wind strength can strongly affect the duration and amplitude of dipole blocking. Thus, while the meridional distribution of the background zonal wind can affect the asymmetry of the blocking dipole in the meridional direction, the duration and strength of the blocking seem to be mainly determined by the magnitude of the meridionally averaged background zonal wind (or PV gradient).

\section{North-south asymmetry of dipole blocking and its physical cause}

In this section, we define $\psi_{A}=\left|\left(\psi_{B}\right)_{\text {Max }}\right|-\left|\left(\psi_{B}\right)_{\text {Min }}\right|$ as a parameter reflecting the meridional asymmetry of the eddy-driven blocking dipole in different westerly wind environments. As shown in Fig. 2b, because the background zonal wind over the North Atlantic is generally weaker in high latitudes and stronger in midlatitudes, this allows us to infer that the meridional asymmetry of the North Atlantic blocking dipole is likely related to the asymmetric distribution of the background zonal wind or the associated PV gradient in the meridional direction. In the following discussions, we further consider three cases and compare their differences to help understand why blocking dipole patterns with a strong anticyclonic anomaly and weak cyclonic anomaly can often occur in the North Atlantic (Fig. 2a).

\section{a. Physical cause of dipole blocking with a strong anticyclonic anomaly and a weak cyclonic anomaly}

Here, we consider $U=u_{0}+\Delta u e^{-\gamma\left(y-y_{0}\right)^{2}} \cos \left\{\left[2 \pi /\left(L_{y}+\right.\right.\right.$ $\left.\left.\left.y_{1}\right)\right] y\right\}$ to represent a southward-shifted jet similar to that in Fig. 2b, where $u_{0}=0.7, \gamma=0.1, y_{0}=1.5$, and $y_{1}=3.0$. For $\Delta u=0.2$ and other parameters listed in Table 1, we show the instantaneous planetary-scale streamfunction $\psi_{P}$, anomaly streamfunction $\psi_{B}$, synoptic-scale 


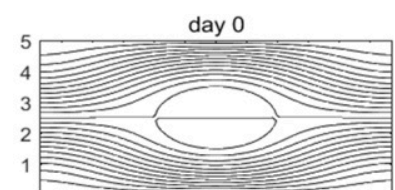

$\begin{array}{llllllllllll}-5 & -4 & -3 & -2 & -1 & 0 & 1 & 2 & 3 & 4 & 5\end{array}$ day 6

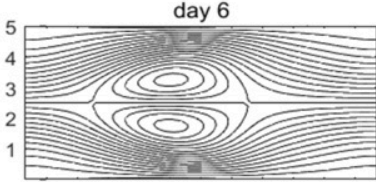

$\begin{array}{lllllllllll}-5 & -4 & -3 & -2 & -1 & 0 & 1 & 2 & 3 & 4 & 5\end{array}$

day 12

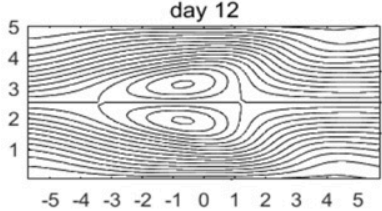

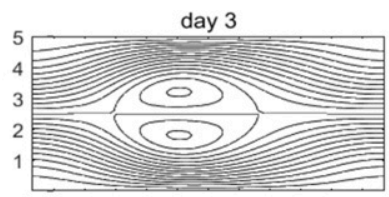

$\begin{array}{llllllllllll}-5 & -4 & -3 & -2 & -1 & 0 & 1 & 2 & 3 & 4 & 5\end{array}$

day 9

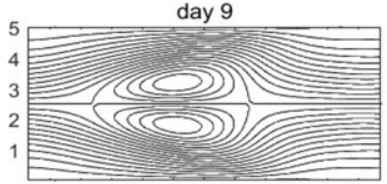

$\begin{array}{lllllllllll}-5 & -4 & -3 & -2 & -1 & 0 & 1 & 2 & 3 & 4 & 5\end{array}$

day 15

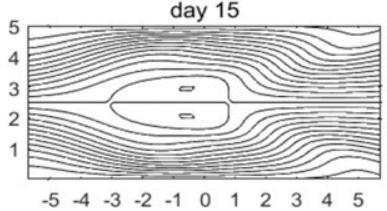

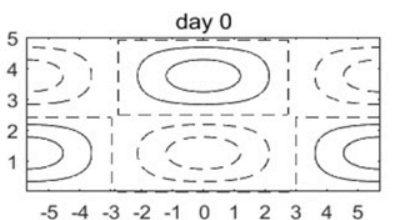

day 6

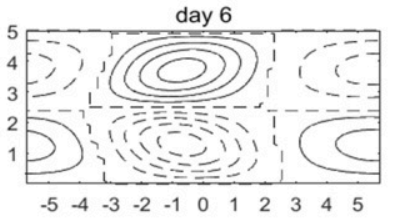

day 12

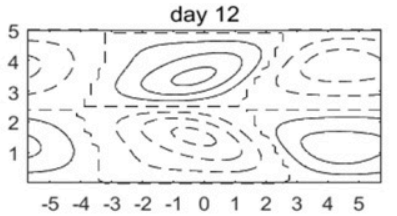

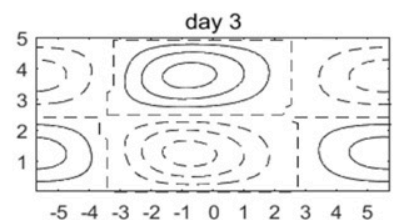

day 9
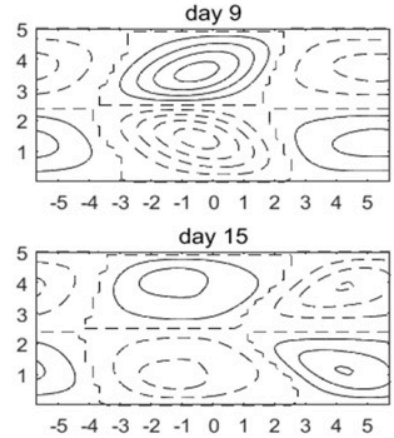

(a)

(b)
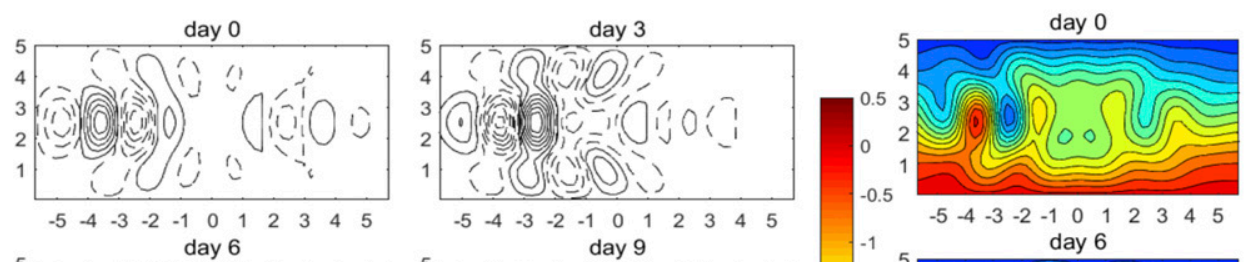

day 9
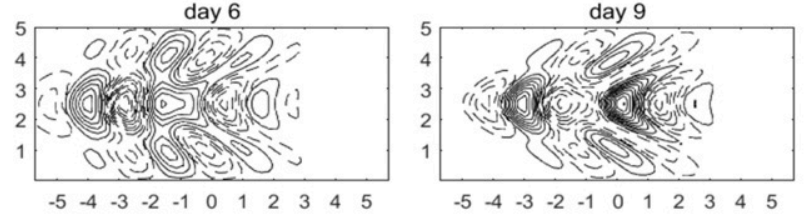

day 12
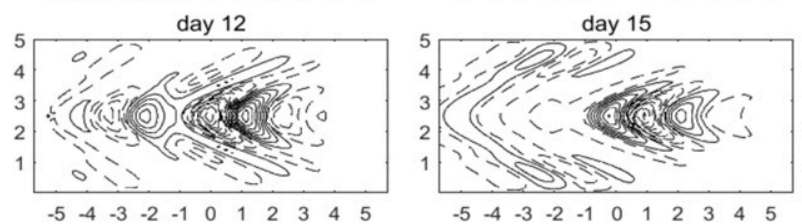

(c)
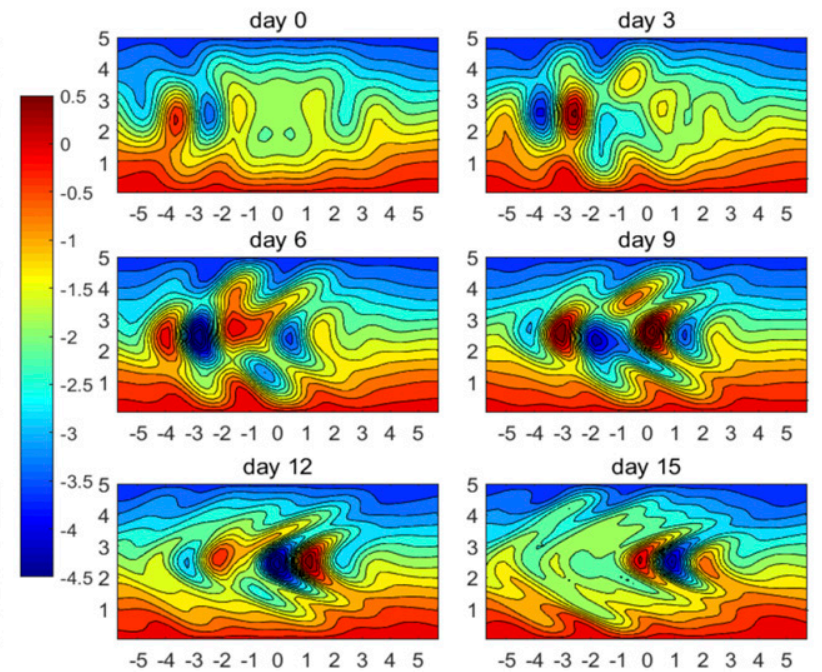

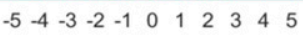
day 15

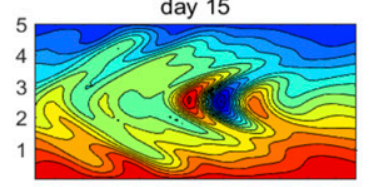

$\begin{array}{lllllllllll}-5 & -4 & -3 & -2 & -1 & 0 & 1 & 2 & 3 & 4 & 5\end{array}$

(d)

FIG. 8. Temporal evolutions of instantaneous (a) planetary-scale streamfunction $\psi_{P}(\mathrm{CI}=0.15)$, (b) blocking wavy anomaly $\psi_{B}(\mathrm{CI}=0.2)$, (c) synoptic-scale streamfunction $\psi^{\prime}(\mathrm{CI}=0.3)$, and $(\mathrm{d})$ total streamfunction $\psi_{T}(\mathrm{CI}=0.3)$ fields during the life cycle of an eddy-driven dipole blocking for the initial amplitude of $B(x, 0)=0.4$ with $\mathrm{PV}_{y}=\beta-U_{y y}+F U, U=u_{0}+\Delta u \cos (m y), u_{0}=0.7$, and $\Delta u=0.2$.

streamfunction $\psi^{\prime}$, and total streamfunction $\psi_{T}$ fields in Fig. 11. It is found that the northern pole of the eddydriven blocking is more intense than its southern pole (Fig. 11a). Thus, this blocking wavy anomaly behaves as an asymmetric dipole (Fig. 11b). This dipole also shows a NW-SE-oriented structure with a strong anticyclonic anomaly to the north and a weak cyclonic anomaly to the south (Figs. 11a,b) consistent with reanalysis data (Fig. 2a), which can also be explained by the latitudinal distributions of $\lambda$ and $\delta$ in the blocking system [Eq. (2)] as shown in Fig. 12. Such a meridional asymmetry is more distinct for $\Delta u=0.4$ (not shown) because the background westerly wind is much weaker in high latitudes than in midlatitudes.

We see that the linear dispersion $\lambda$ is weaker in higher latitudes than that in lower latitudes for the given background zonal wind (Fig. 12e), whereas the blocking nonlinearity $\delta$ shows an opposite distribution (Fig. 12f). Thus, more energy can be accumulated in the north side of the blocking dipole than over its south side because of this difference in energy dispersion (Fig. 12e) or nonlinearity (Fig. 12f). Such a distribution of energy accumulation helps explain why the northern pole of the blocking is stronger than the southern pole 
(a)

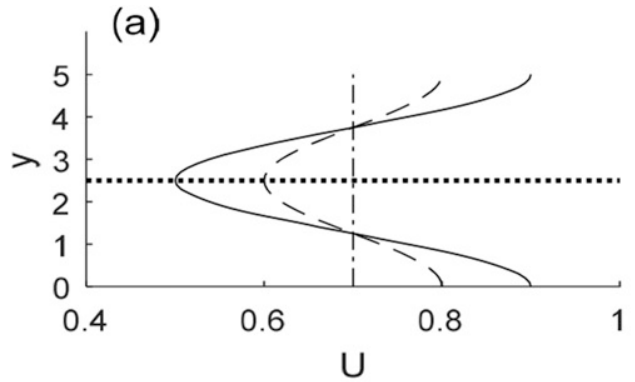

(c)

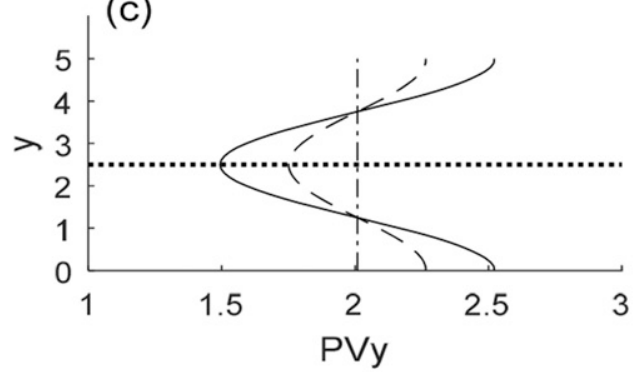

(e)

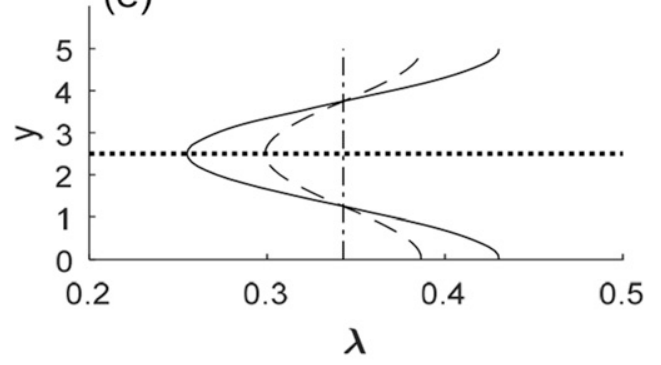

(b)

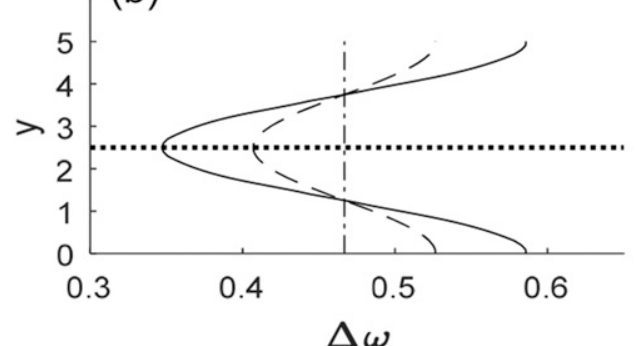

(d)

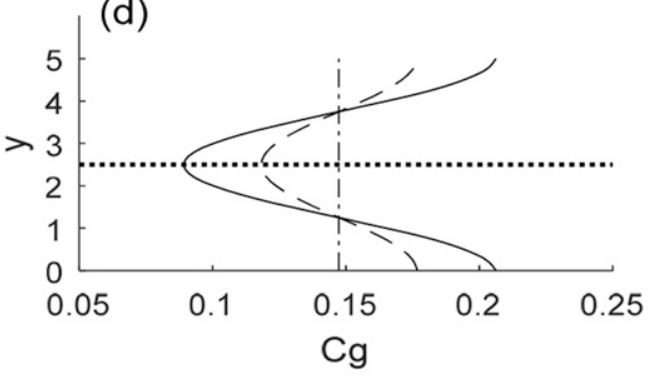

(f)

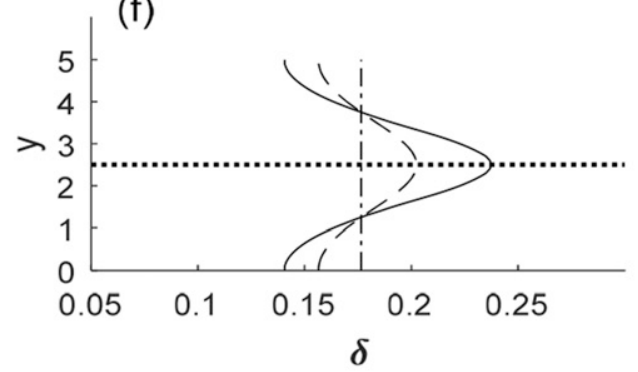

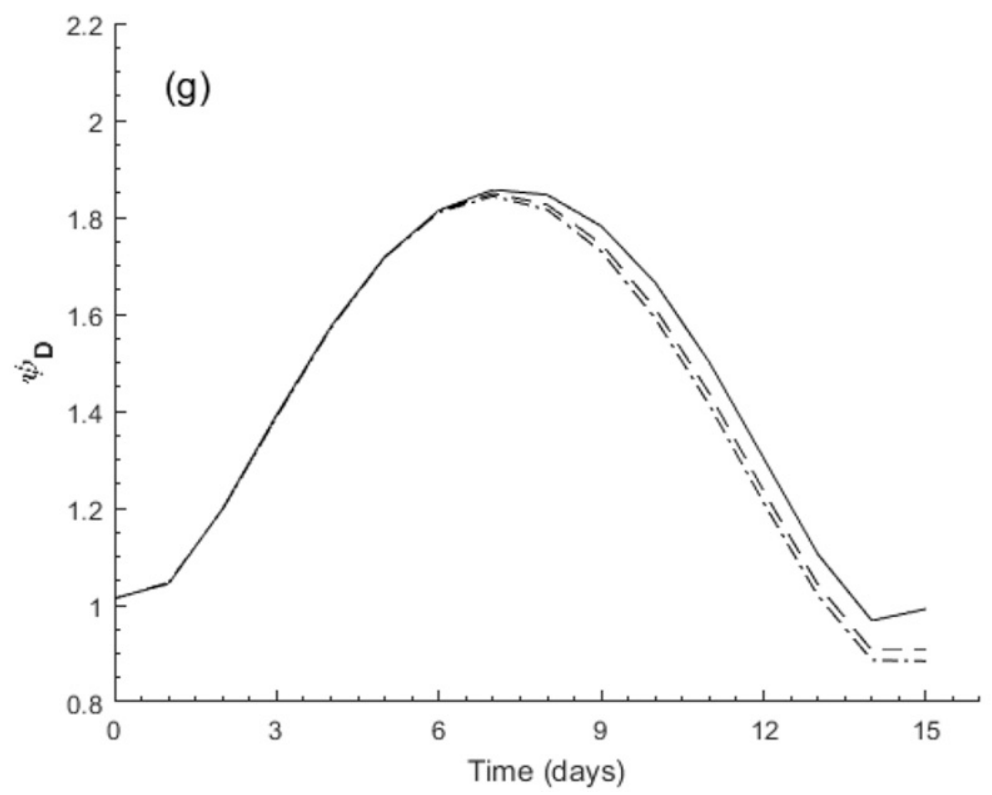

FIG. 9. Meridional distributions of (a) $U$, (b) $\Delta \omega$, (c) $\mathrm{PV}_{y}$, (d) $C_{g}$, (e) $\lambda$, and (f) $\delta$ for three cases: $\Delta u=0$ (dotdashed line), 0.1 (long-dashed line), and 0.2 (solid line) of $U=u_{0}+\Delta u \cos (m y)$ and $u_{0}=0.7$. (g) Time series of daily blocking amplitude $\psi_{D}$ during the blocking life cycle for $\Delta u=0$ (dot-dashed line), 0.1 (long-dashed line) and 0.2 (solid line). The dotted line in (a)-(f) denotes the central latitude of $y=2.5$. 

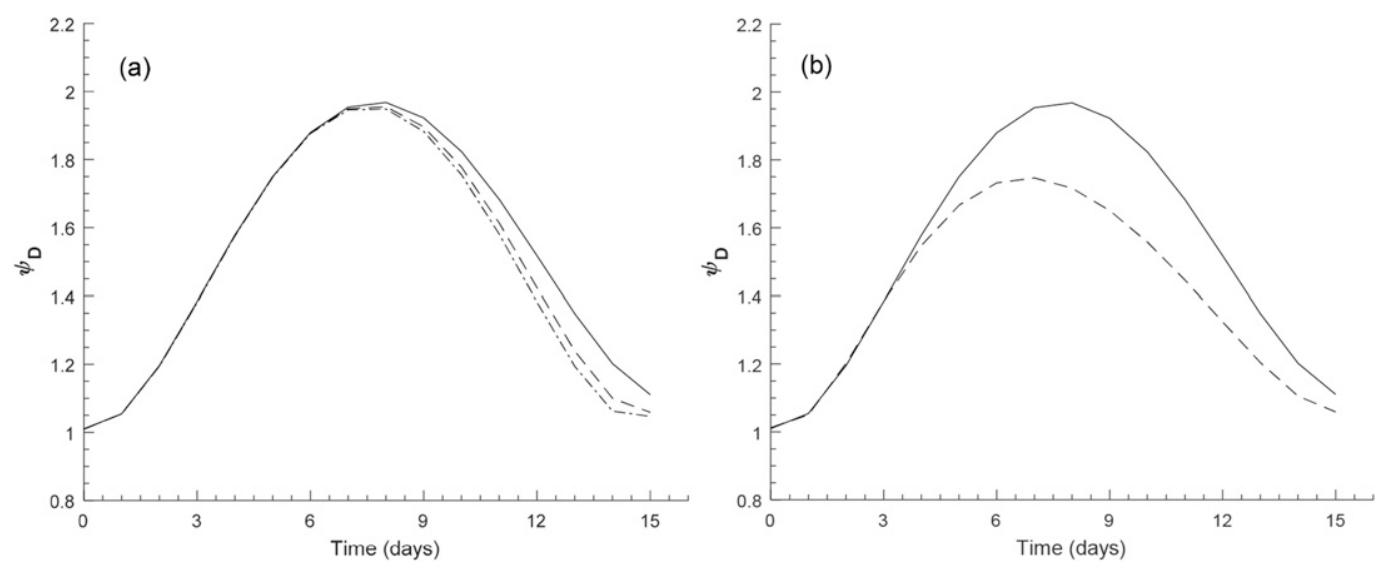

FIG. 10. (a) Time series of daily blocking amplitude $\psi_{D}$ of an eddy-driven blocking with $U=u_{0}+\Delta u \cos (m y)$ and $u_{0}=0.5$ during the blocking life cycle for $\Delta u=0$ (dot-dashed line), 0.1 (long-dashed line) and 0.2 (solid line). (b) Daily time variations of the blocking amplitude $\psi_{D}$ with $\Delta u=0.2$ for $u_{0}=0.5$ (solid line) and $u_{0}=0.9$ (dashed line).

(Figs. 11a,b). Figures $12 \mathrm{~g}$ and $12 \mathrm{~h}$ show that while the strength and duration of the dipole blocking do not change with the value of $\Delta u$, its meridional asymmetry is intensified with the growth of the blocking and also more distinct for larger values of $\Delta u$ (Fig. 12h). Such an asymmetry is more distinct during the blocking decay phase than during its growth phase. This may be because the meridional asymmetry of the blocking amplitude is enhanced after the blocking peak because of enhanced energy dispersion of the southern pole of the blocking dipole. However, the observed blocking asymmetry is also related to many other factors such as the meridional asymmetry of the incipient blocking dipole, baroclinicity, and the barotropic decay of upstream traveling synoptic-scale eddies. A strong meridional asymmetry of blocking dipole can also be seen during the blocking growing phase if the initial blocking dipole has a large meridional asymmetry. For example, we consider an initial value as $B(x, y, 0)=0.4 \exp \left[-\sigma \varepsilon^{2}(y-\right.$ $\left.\left.y_{0}\right)^{2}\right]$, where $y_{0}=3.75$ and $\sigma$ is a constant. Here, $\sigma=0$ corresponds to the case of Fig. 11, but $\sigma=2.0$ represents an initial blocking dipole having a large meridional asymmetry. For $\sigma=2.0$ and the other parameters same as in Fig. 11, we show the instantaneous fields of the blocking wavy anomaly $\psi_{B}$ and total streamfunction $\psi_{T}$ of eddy-driven dipole blocking and the time series of the blocking intensity $\psi_{D}$ and meridional asymmetry $\psi_{A}$ in Fig. 13. It is found that while the total streamfunction field (Fig. 13b) of blocking still resembles that in Fig. 11d, their details are slightly different. For an initial blocking dipole with a large meridional asymmetry, the meridional asymmetry of eddy-driven blocking dipole is further intensified with the blocking growth (Fig. 13a). In particular, the blocking asymmetry (Fig. 13d) becomes more intense during the blocking growing phase than during the blocking decaying phase and is more consistent with the reanalysis data result (Fig. 2a), even though the blocking intensity is slightly weaker for $\sigma=2.0$ than for $\sigma=0$ (Fig. 13c). This indicates that the meridional asymmetry of the initial blocking is important for the asymmetry of the subsequent blocking dipole.

To account for the NW-SE orientation, it is useful to examine the meridional variations of linear phase speed $C_{p}$, blocking-induced speed $C_{N}$, and nonlinear phase speed $C_{\mathrm{NP}}$ during the blocking life cycle (Fig. 14). In the $C_{p}, C_{N}$, and $C_{\mathrm{NP}}$ expressions, the meridional distribution of $C_{\mathrm{NP}}$ can reflect whether the blocking dipole shows a horizontal orientation or not. It is found that the linear phase speed $C_{p}$ of the blocking dipole is more negative in the north side of the central latitude $y=2.5$ than in the south side (Fig. 14a). Since the blocking dipole moves with the nonlinear phase speed $C_{\mathrm{NP}}=C_{p}+C_{N}$, it would move more westward over its north side than over its south side when $C_{\mathrm{NP}}$ has a larger negative value over the north side than over the south side. The zonal movement of the blocking dipole depends mainly on the background westerly wind if the blocking amplitude $M_{0}$ is small and thus $C_{N}=-\delta_{N} M_{0}^{2} /\left(2 k \mathrm{PV}_{y}\right) \approx 0$. However, $C_{N}$ becomes more important as the blocking amplitude increases to a larger value (Fig. 14b), but the net result is that there is a tendency of larger negative $C_{\mathrm{NP}}\left(C_{\mathrm{NP}}<0\right)$ in higher latitudes such that the dipole blocking moves westward faster in the high latitudes than in the low latitudes (Fig. 14c). This leads to a horizontal orientation along the NW-SE direction. Of course, such a blocking structure also depends on the spatial distribution of the blocking wave packet amplitude $|B|$ (Fig. 15).

For a background symmetric double jet of $U=$ $u_{0}+\Delta u \cos (m y)$, the blocking amplitude is always 

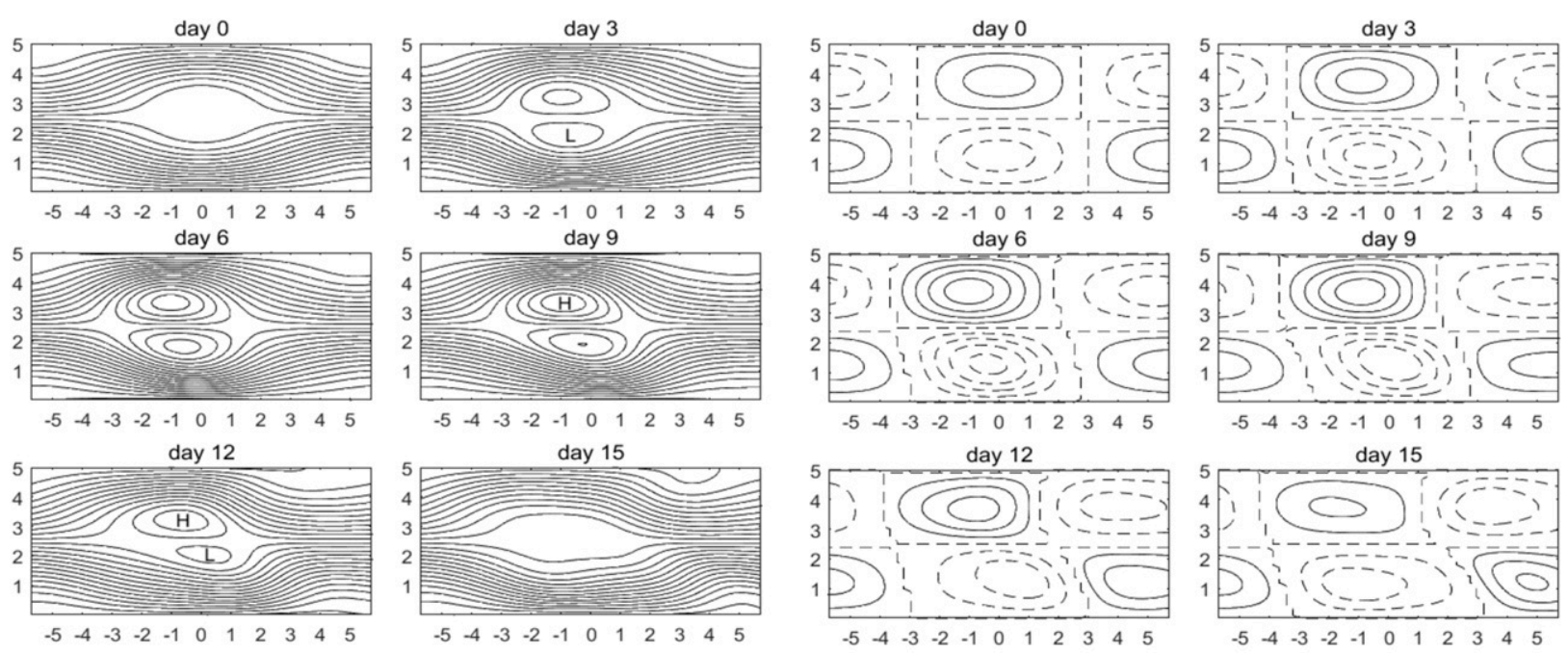

(a)

(b)
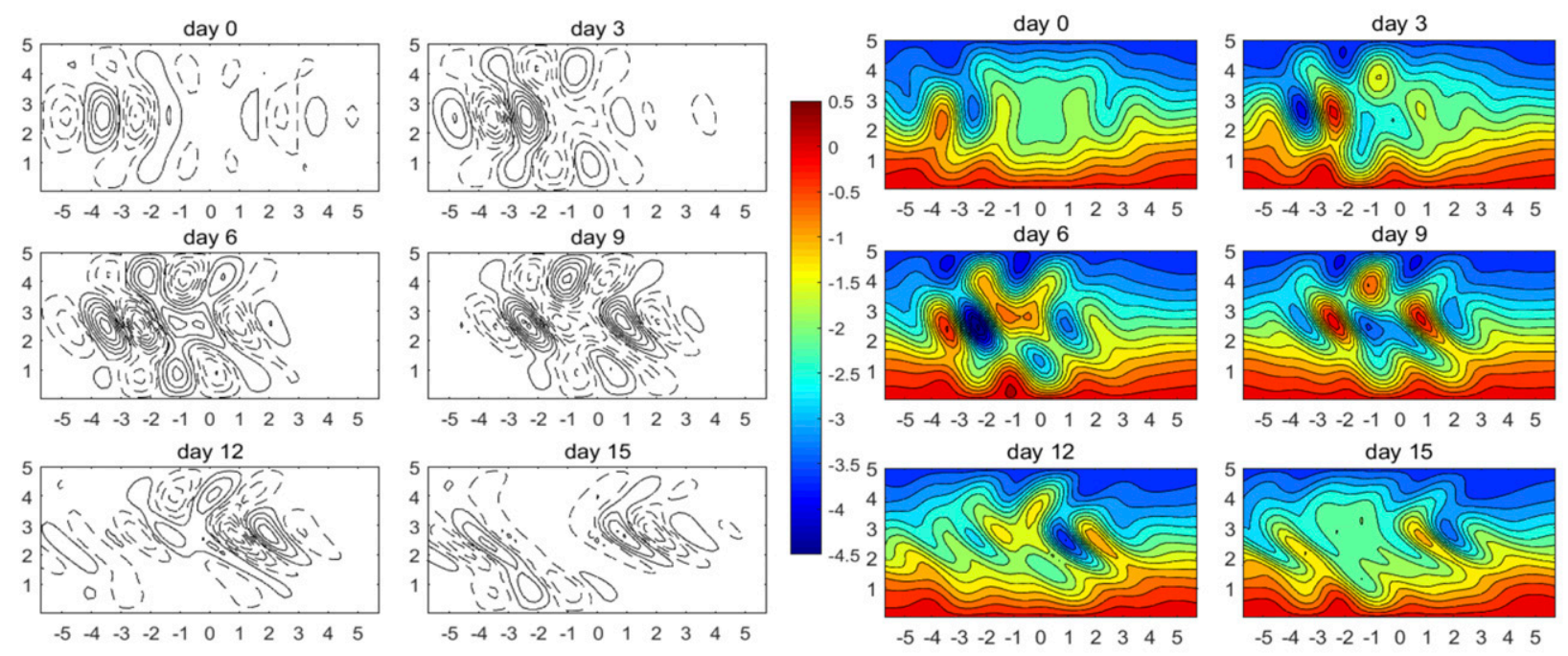

(c)

(d)

FIG. 11. As in Fig. 8, but for $U=u_{0}+\Delta u e^{-\gamma\left(y-y_{0}\right)^{2}} \cos \left\{\left[2 \pi /\left(L_{y}+y_{1}\right)\right] y\right\}, u_{0}=0.7, \Delta u=0.2, \gamma=0.1, y_{0}=1.5$, and $y_{1}=3$.

symmetric with respect to $y=2.5$ during the blocking life cycle (Fig. 15a). Thus, it is inevitable that the dipole blocking does not show a horizontal tilting in the meridional direction (Figs. 8a,b) because the nonlinear phase speed of the dipole blocking possesses a symmetric distribution in the meridional direction (not shown) as the background westerly wind shows a symmetric distribution in the meridional direction. However, for an asymmetric double jet of $U=u_{0}+\Delta u e^{-\gamma\left(y-y_{0}\right)^{2}} \cos \left\{\left[2 \pi /\left(L_{y}+y_{1}\right)\right] y\right\}$, the intensification of the blocking amplitude is mainly concentrated in the north side of the blocking region (Fig. 15b) where the weakened energy dispersion and intensified nonlinearity can take place (Figs. 12e,f). Moreover, because the blocking amplitude is larger over the north side of the channel than over its south side (Fig. 15b), the blocking-induced westward speed is stronger in the north side of blocking region than its south side (Fig. 14c). The combination of $C_{p}$ and $C_{N}$ leads to a large negative value of $C_{\mathrm{NP}}$ in the north side of the blocking dipole and a small negative or positive value over its south side (Fig. 14c). Such a nonlinear phase speed distribution leads to the NW-SE orientation of blocking dipole (Figs. 11a,b). This can explain why the observed North Atlantic blocking dipole shows a strong meridional asymmetry and a NW-SE orientation (Fig. 2a). To test the sensitivity of our results to different background zonal winds, we also calculated 
(a)

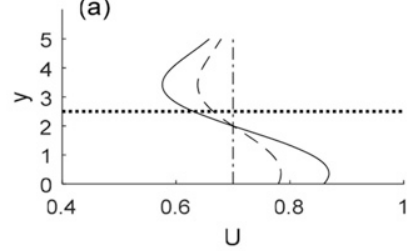

(c)

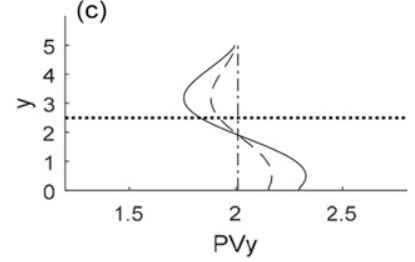

(e)
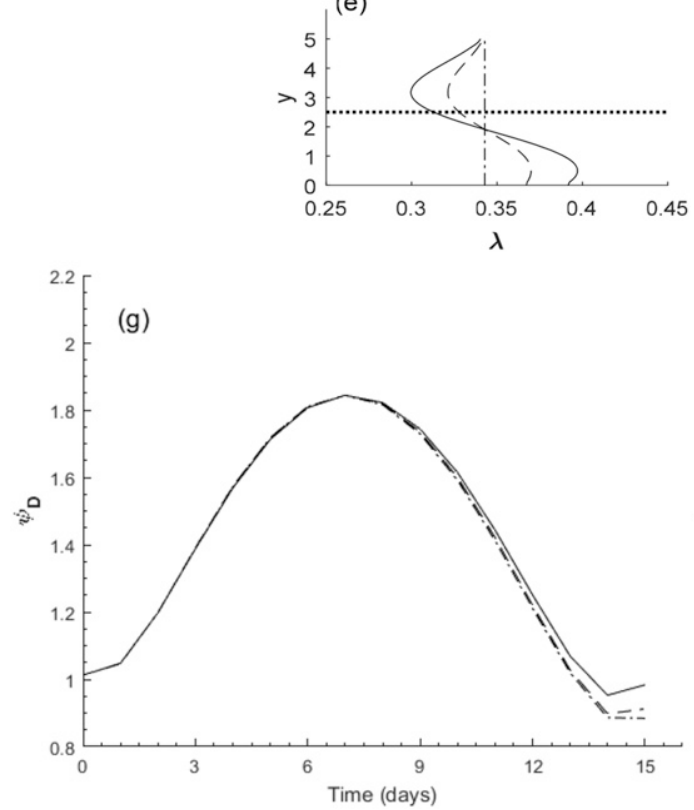

(b)

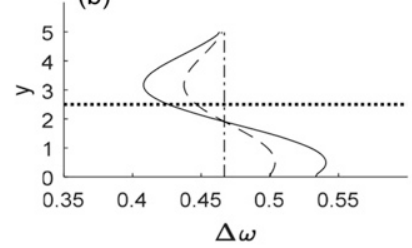

(d)
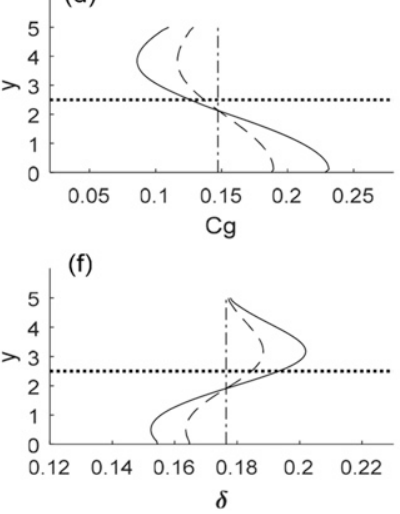

(h)

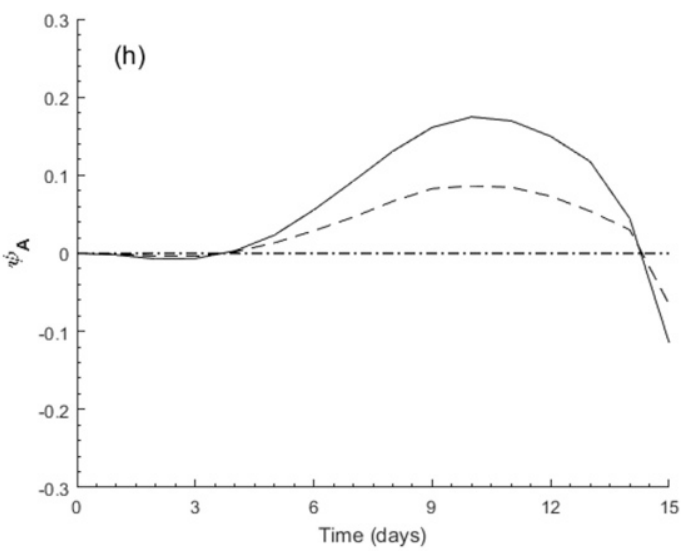

FIG. 12. (top) Meridional distributions of (a) $U$, (b) $\Delta \omega$, (c) $\mathrm{PV}_{y}$, (d) $C_{g}$, (e) $\lambda$, and (f) $\delta$ for three cases: $\Delta u=0$ (dotdashed line), 0.1 (long-dashed line), and 0.2 (solid line) of $U=u_{0}+\Delta u e^{-\gamma\left(y-y_{0}\right)^{2}} \cos \left\{\left[2 \pi /\left(L_{y}+y_{1}\right)\right] y\right\}, u_{0}=0.7$, $\gamma=0.1, y_{0}=1.5$, and $y_{1}=3$. (bottom) Time series of (g) daily blocking dipole amplitude $\psi_{D}$ and (h) asymmetry $\psi_{A}\left[\psi_{A}=\left|\left(\psi_{B}\right)_{\text {Max }}\right|-\left|\left(\psi_{B}\right)_{\text {Min }}\right|\right]$ during the blocking life cycle for $\Delta u=0$ (dot-dashed line), 0.1 (long-dashed line), and 0.2 (solid line). The dotted line in (a)-(f) denotes the central latitude of $y=2.5$.

the case of $U=u_{0}+\Delta u e^{-\gamma\left(y-y_{0}\right)^{2}}$ for $\gamma>0$. A similar result is found (not shown).

The above results clearly reveal that the northsouth asymmetry of the eddy-driven dipole blocking is significantly influenced by the meridional distribution of the background zonal wind. More specifically, weak (strong) background westerly winds in the higher (lower) latitudes favor a NW-SE-oriented dipole blocking with a strong anticyclonic anomaly to the northwest and a weak cyclonic anomaly to the southeast of the central latitude of the blocking region because of reduced (enhanced) energy dispersion and increased (decreased) nonlinearity in the higher (lower) latitudes. b. The physical cause of dipole blocking with a weak anticyclonic anomaly and a strong cyclonic anomaly

Here, we still assume that the mathematical form of background zonal wind is same as $U=u_{0}+\Delta u e^{-\gamma\left(y-y_{0}\right)^{2}} \times$ $\cos \left\{\left[2 \pi /\left(L_{y}+y_{1}\right)\right] y\right\}$, but allow the background zonal winds to be strong in higher latitudes. For $\Delta u=-0.2$ of $U=u_{0}+\Delta u e^{-\gamma\left(y-y_{0}\right)^{2}} \cos \left\{\left[2 \pi /\left(L_{y}+y_{1}\right)\right] y\right\}$ with the other parameters described in Fig. 11a, the instantaneous $\psi_{P}, \psi_{B}, \psi^{\prime}$, and $\psi_{T}$ fields of an eddy-driven dipole blocking event and the variations of corresponding $U, \Delta \omega$, $\mathrm{PV}_{y}, C_{g}, \lambda$, and $\delta$ with the latitude are shown in Figs. 16 and 17 , respectively. It is seen that the eddy-driven blocking 

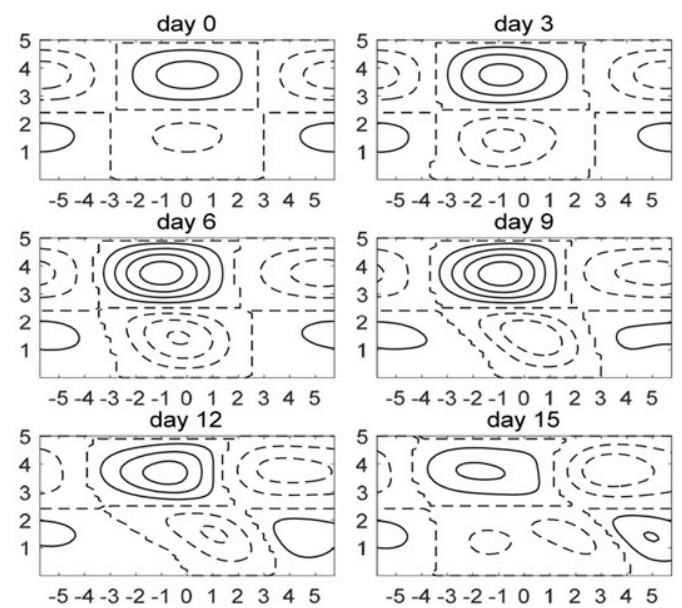

(a)

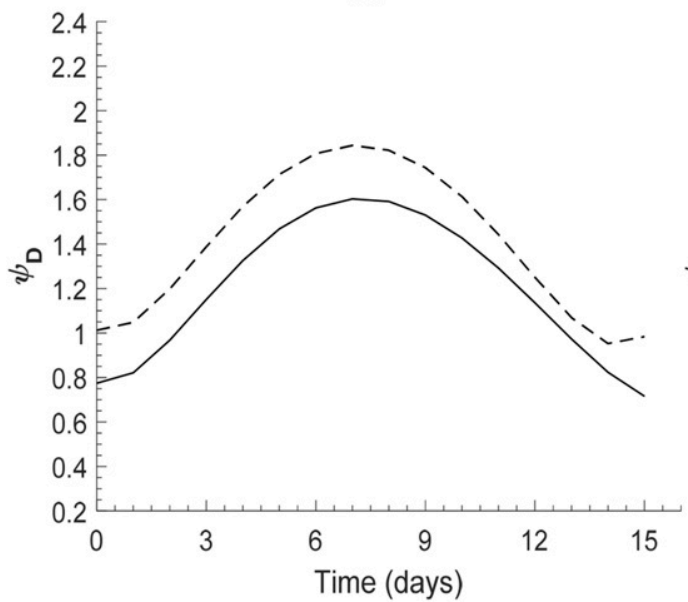

(c)
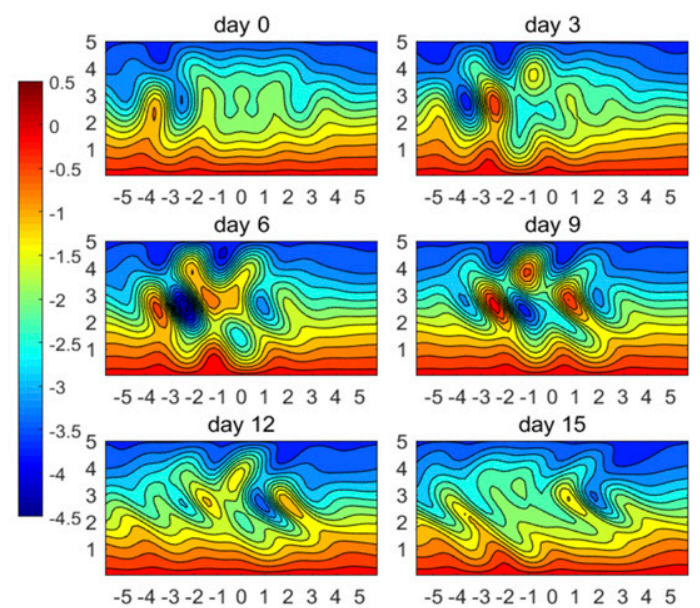

day 9
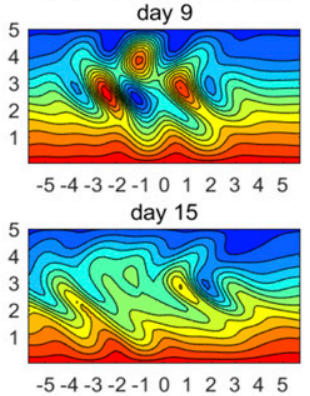

(b)

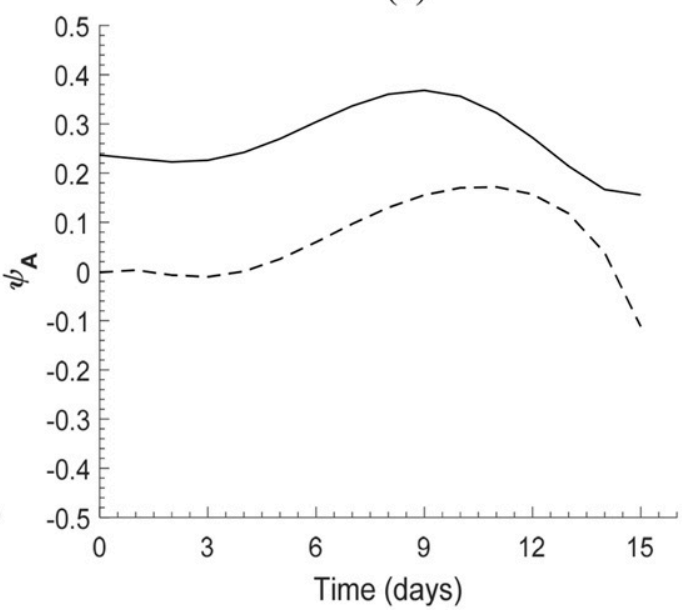

(d)

FIG. 13. (top) Instantaneous (a) blocking wavy anomaly $\psi_{B}(\mathrm{CI}=0.2)$ and (b) total streamfunction $\psi_{T}(\mathrm{CI}=0.3)$ fields of an eddy-driven dipole blocking. (bottom) Time series of (c) blocking intensity $\psi_{D}$ and (d) meridional asymmetry $\psi_{A}$ for the initial amplitude of $B(x, y, 0)=0.4 \exp \left[-\sigma \varepsilon^{2}\left(y-y_{0}\right)^{2}\right]$ with $y_{0}=3.75$ and the same parameters as in Fig. 11 for two cases with $\sigma=2.0$ (solid line) and $\sigma=0$ (dashed line).

shows a southwest-northeast (SW-NE)-oriented dipole pattern with a weak anticyclonic anomaly and a strong cyclonic anomaly (Figs. 16a,b), because the energy dispersion of the blocking is intensified and its nonlinearity is weakened in higher latitudes (Figs. 17e,f) as the westerly jet is shifted to higher latitudes (Fig. 17a). Obviously, the north-south asymmetry of blocking dipole is attributed to the meridional asymmetries of its energy dispersion and nonlinearity (Figs. 17e,f) because of the meridional asymmetry of $U$ and $\mathrm{PV}_{y}$ (Figs. 17a,c).

It is also noted that the synoptic-scale eddies are split into two branches, in which the northern branch moves eastward faster than the southern one (Fig. 16c). For this case, a SW-NE-oriented meandering westerly jet stream is seen in the total field (Fig. 16d). The SW-NE orientation of the blocking dipole (Fig. 16b) can be explained by its rapid (slow) eastward movement in higher (lower) latitudes in terms of $C_{\mathrm{NP}}$ (not shown).

While the blocking asymmetry for $\Delta u=-0.2$ (Fig. 17h) has the same amplitude as that for $\Delta u=$ 0.2 (Fig. 12h), they have opposite signs. Here we find that the persistence and strength of dipole blocking is not strongly influenced by the magnitude of $\Delta u$ from $\Delta u=0.2$ to $\Delta u=-0.2$, even though the amplitude of the northern pole is different. A possible cause of it is that the change of the meridionally averaged $\mathrm{PV}_{y}$ is small between $\Delta u=0.2$ and $\Delta u=-0.2$.

The above results reveal that while the magnitude of the meridionally averaged $\mathrm{PV}_{y}$ is important for the persistence and strength of dipole blocking, the meridional distribution of the background zonal winds can 


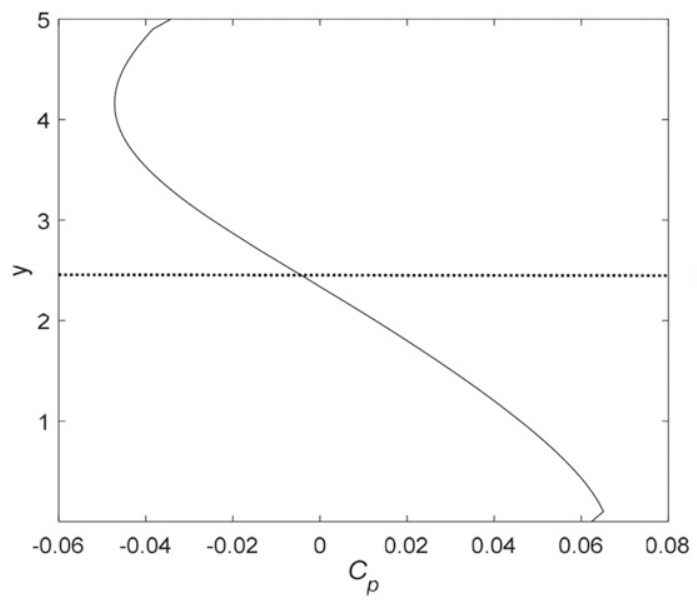

(a)

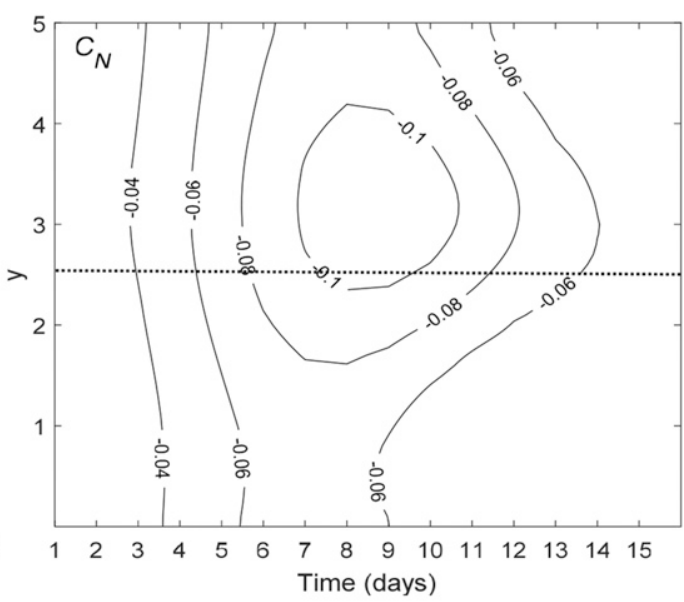

(b)

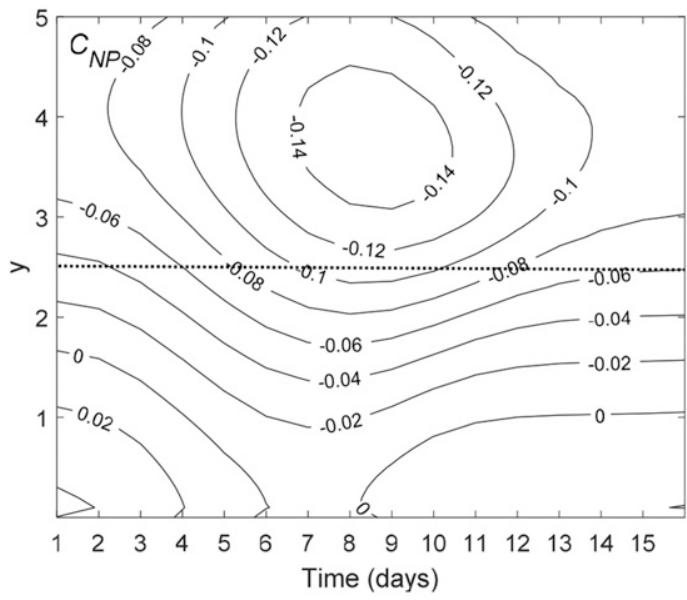

(c)

FIG. 14. (a) Meridional distribution of the linear phase speed $C_{p}$ of the dipole blocking. (b),(c) Time-latitude evolution of (b) blocking-induced westward speed $C_{N}$ and (c) nonlinear phase speed $C_{\mathrm{NP}}$ of eddy-driven dipole blocking in a given background zonal wind $U$ shown in Fig. 11, where $C_{\mathrm{NP}}=C_{p}+C_{N}$. The blocking dipole moves with the speed of $C_{\mathrm{NP}}$ and the dotted line denotes the central latitude of $y=2.5$.

significantly affect the north-south asymmetry of the eddy-driven dipole blocking through different meridional distributions of the movement speed, energy dispersion, and nonlinearity related to the meridional distribution of $\mathrm{PV}_{y}$. The blocking asymmetry becomes more evident when the background zonal winds have a stronger asymmetric jetlike structure shifting from higher to lower latitudes or conversely.

\section{Impacts of background zonal wind and its nonuniform meridional shear on the blocking dipole}

As we have noted above, the change of $\mathrm{PV}_{y}=\beta-$ $U_{y y}+F U$ is mainly influenced by $U$ and $U_{y y}$ for a fixed $\beta$. Thus, to examine the different effects of the background zonal wind $U$ and its nonuniform shear $U_{y y}$ on the eddydriven dipole blocking, we consider two cases: one is $\mathrm{PV}_{y}=\beta+F U$ without $U_{y y}$ and another is $\mathrm{PV}_{y}=\beta-U_{y y}$ without the background zonal wind strength $U$.

For $U=u_{0}+\Delta u e^{-\gamma\left(y-y_{0}\right)^{2}} \cos \left\{\left[2 \pi /\left(L_{y}+y_{1}\right)\right] y\right\}$ with the same parameters as in Fig. 11, we show the planetaryscale streamfunction $\left(\psi_{P}\right)$ field of the eddy-driven dipole blocking and its intensity $\psi_{D}$ and asymmetry $\psi_{A}$ in Fig. 18. It is seen that the blocking dipole has larger amplitude and longer lifetimes for $\mathrm{PV}_{y}=\beta-U_{y y}$ (Fig. 18b) than for $\mathrm{PV}_{y}=\beta+F U$ (Fig. 18a). The blocking moves eastward for $\mathrm{PV}_{y}=\beta-U_{y y}$ (Fig. 18b), but westward for $\mathrm{PV}_{y}=\beta+F U$ (Fig. 18a). Because the presence of $U\left(U_{y y}\right)$ tends to enhance (reduce) the PV gradient for $U_{y y}>0$, the role of $U_{y y}>0$ can increase the 

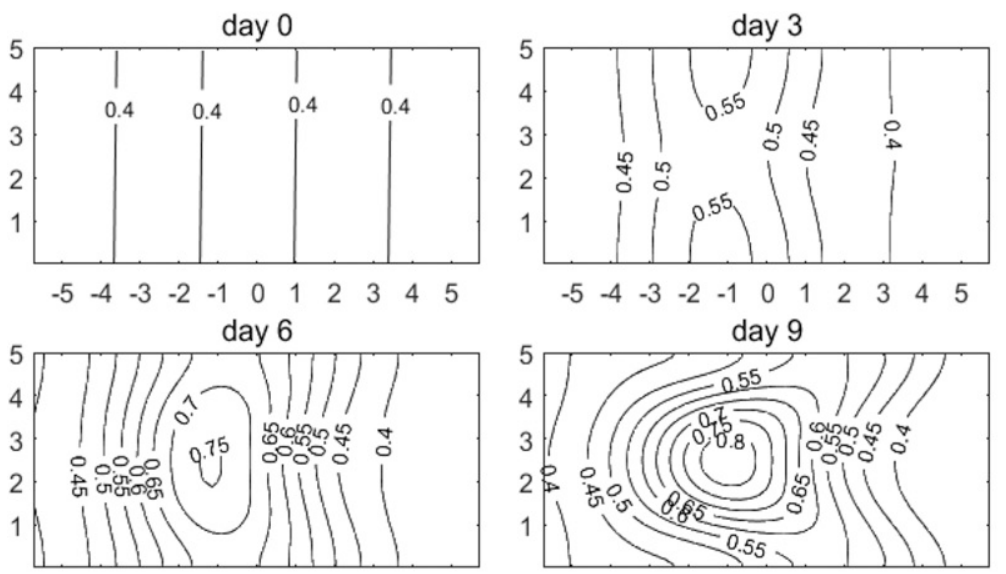

$\begin{array}{lllllllllll}-5 & -4 & -3 & -2 & -1 & 0 & 1 & 2 & 3 & 4 & 5\end{array}$
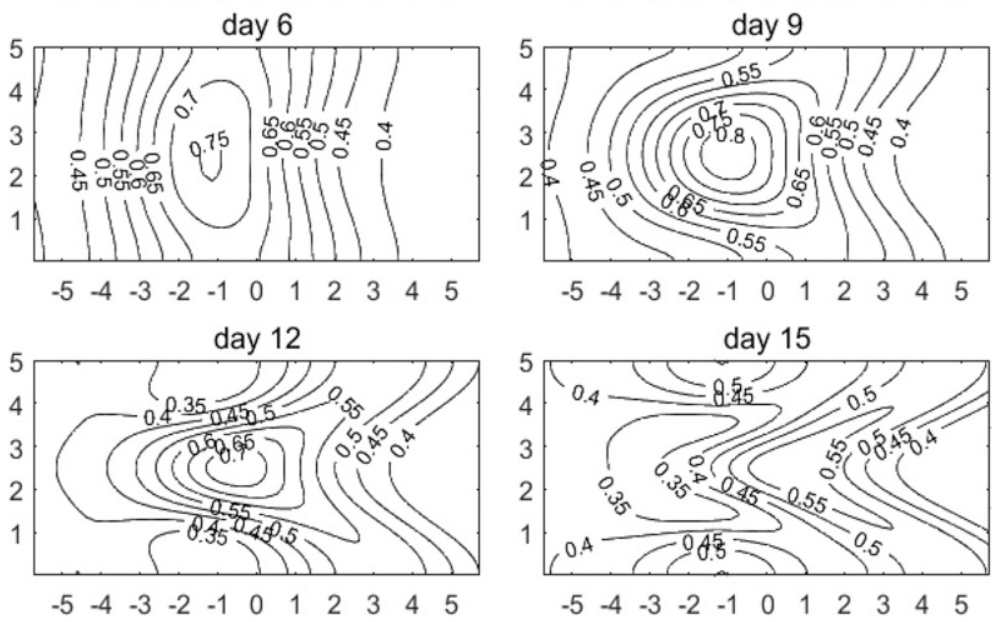

(a)
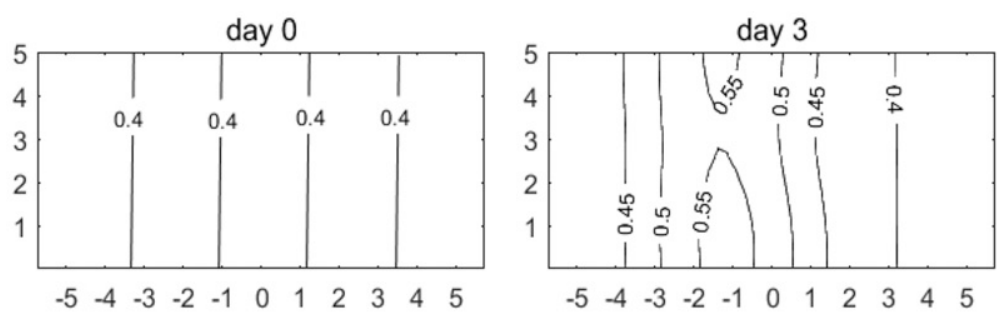

day 6
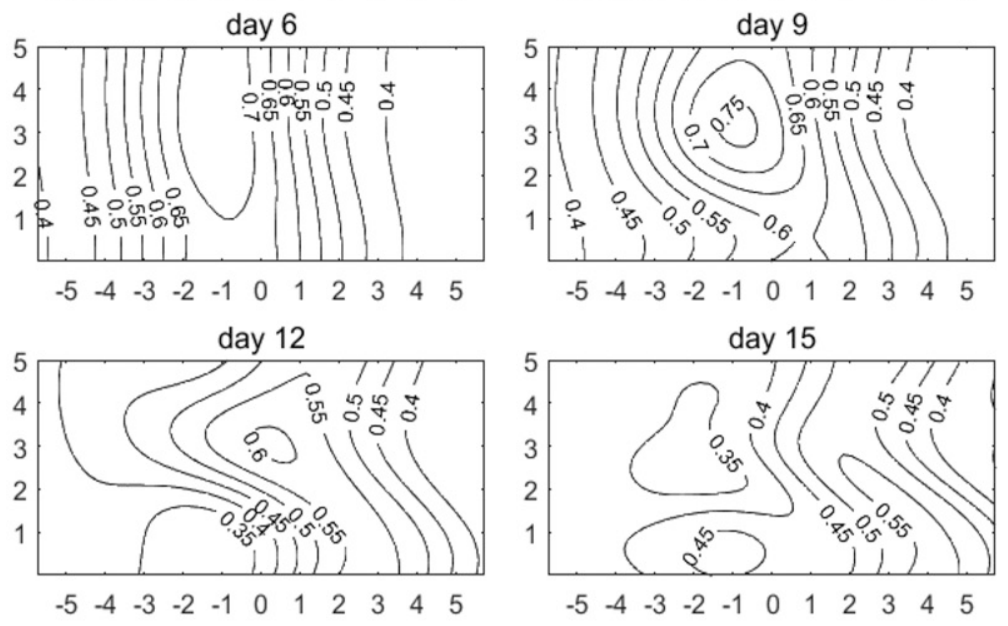

(b)

FIG. 15. Time sequences of the blocking wave packet amplitude $|B(x, y, t)|$ of an eddy-driven dipole blocking for given background zonal winds: (a) $U=u_{0}+\Delta u \cos (m y)$ with $u_{0}=0.7$ and $\Delta u=0.2$ and (b) $U=u_{0}+\Delta u e^{-\gamma\left(y-y_{0}\right)^{2}} \cos \left\{\left[2 \pi /\left(L_{y}+y_{1}\right)\right] y\right\}$ with $u_{0}=0.7, \Delta u=0.2, \gamma=0.1, y_{0}=1.5$, and $y_{1}=3$. 

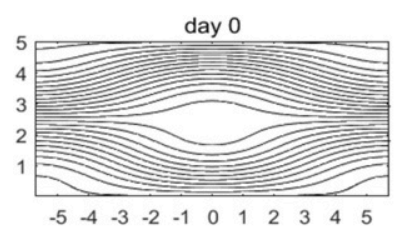
day 6

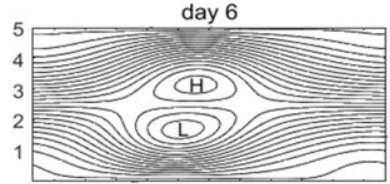

$\begin{array}{lllllllllll}-5 & -4 & -3 & -2 & -1 & 0 & 1 & 2 & 3 & 4 & 5\end{array}$

day 12

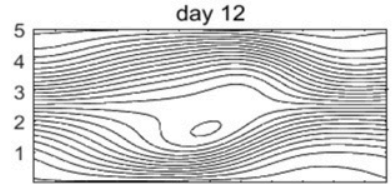

$\begin{array}{lllllllllll}-5 & -4 & -3 & -2 & -1 & 0 & 1 & 2 & 3 & 4 & 5\end{array}$

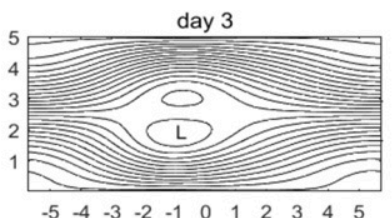

day 9

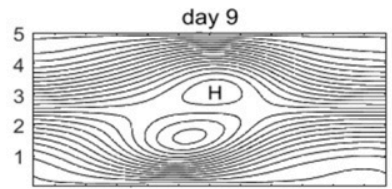

$\begin{array}{lllllllllll}-5 & -4 & -3 & -2 & -1 & 0 & 1 & 2 & 3 & 4 & 5\end{array}$

day 15

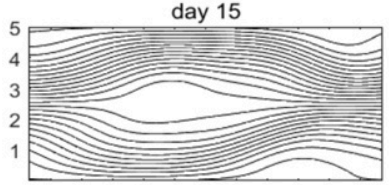

$\begin{array}{llllllllllll}-5 & -4 & -3 & -2 & -1 & 0 & 1 & 2 & 3 & 4 & 5\end{array}$
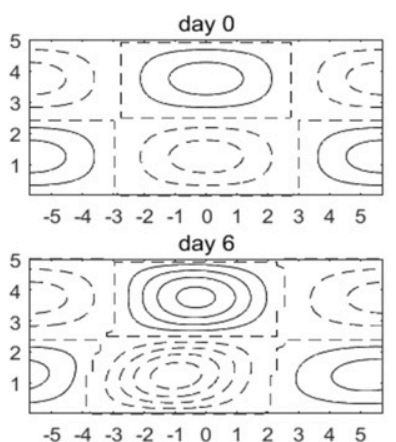

day 12

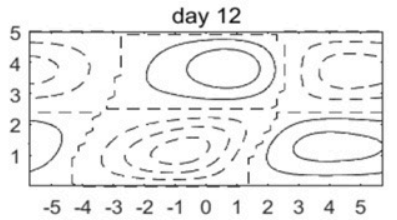

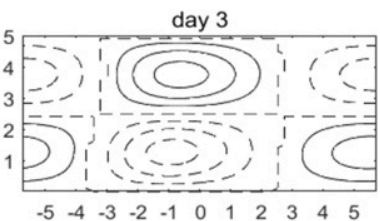

day 9

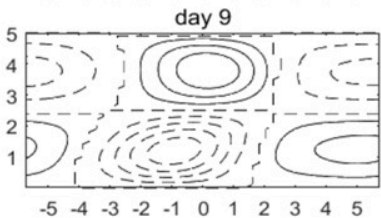

day 15

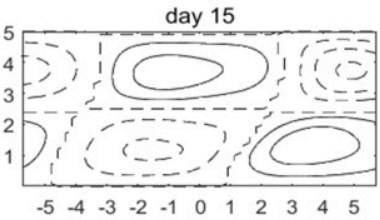

(b)

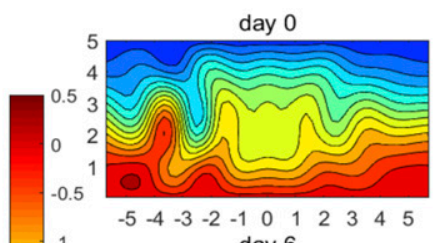

day 9

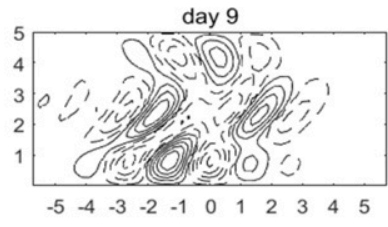

day 15

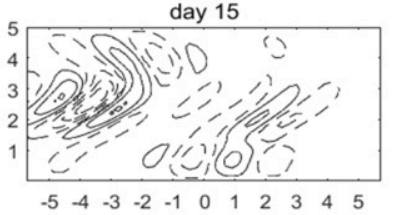

$\begin{array}{lllllllllll}-5 & -4 & -3 & -2 & -1 & 0 & 1 & 2 & 3 & 4 & 5\end{array}$ day 6

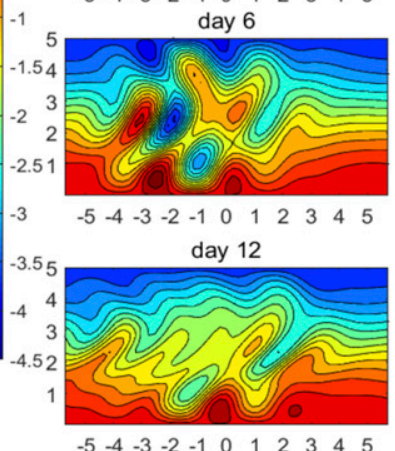

$\begin{array}{lllllllllll}-5 & -4 & -3 & -2 & -1 & 0 & 1 & 2 & 3 & 4 & 5\end{array}$

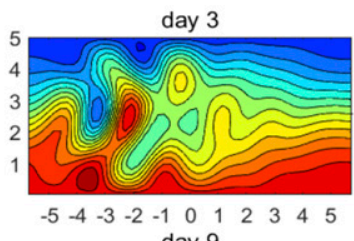

day 9

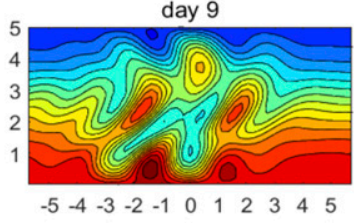

day 15

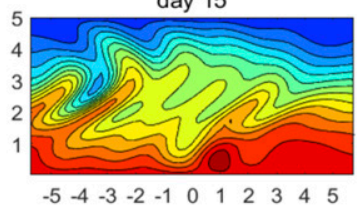

(c)

(d)

FIG. 16. As in Fig. 11, but for $U=u_{0}+\Delta u e^{-\gamma\left(y-y_{0}\right)^{2}} \cos \left\{\left[2 \pi /\left(L_{y}+y_{1}\right)\right] y\right\}$ with $u_{0}=0.7, \Delta u=-0.2, \gamma=0.1, y_{0}=1.5$, and $y_{1}=3$.

lifetime and eastward movement of dipole blocking compared to the effect of weakened $U$. In other words, the nonuniform meridional shear of the background zonal wind is more important for the intensity, movement and persistence time of dipole blocking than under this $U$ distribution (Fig. 12a). This can be further seen from the time variation of the strength of dipole blocking as shown in Fig. 18c, while the difference of the meridional asymmetry of blocking dipole between the two cases is less distinct before the blocking decay (Fig. 18d). The above result also holds for other types of background zonal winds and is insensitive to the different choice of $F$ (not shown). It is worth pointing out that when the Barents-Kara Seas experience a large warming, the change of $U_{y y}$ may be much larger than the change in the strength of $U$ in some regions (Luo et al. 2018). Thus, the magnitude of $\mathrm{PV}_{y}$ is a more appropriate indicator of the blocking change, especially for detecting omega-type blocking events, than $U$ because $\mathrm{PV}_{y}$ combines $U_{y y}$ and $U$ into a single index.

\section{Conclusions and discussion}

In this paper, an extended the nonlinear multiscale interaction (NMI) or ENMI model of blocking events including slowly varying background zonal winds is used to examine how the meridional distribution of the 
(a)

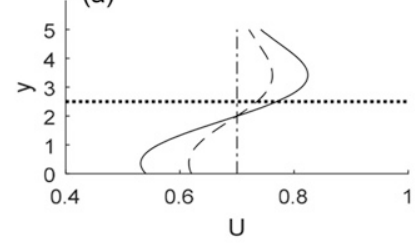

(c)

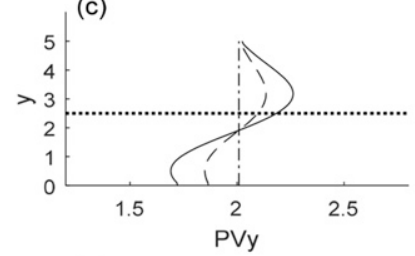

(e)

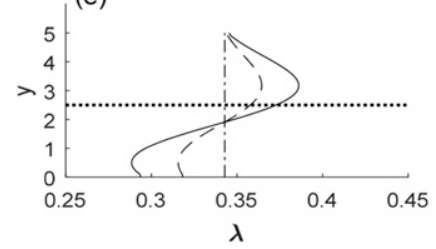

(g)

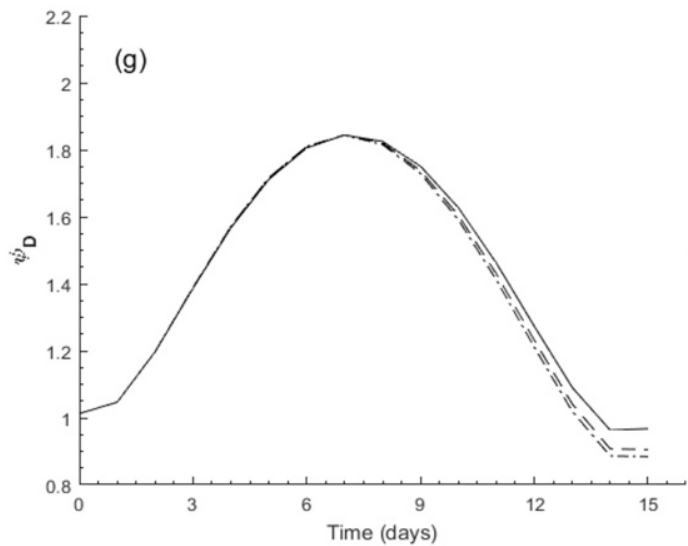

(b)

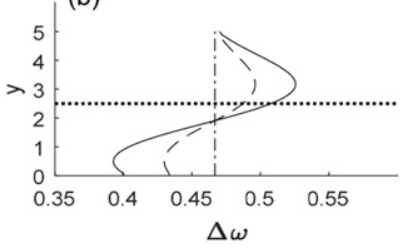

(d)

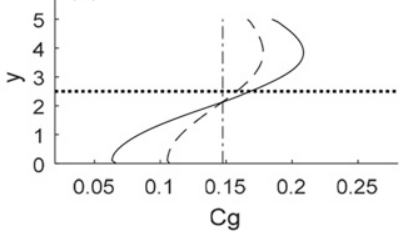

(f)

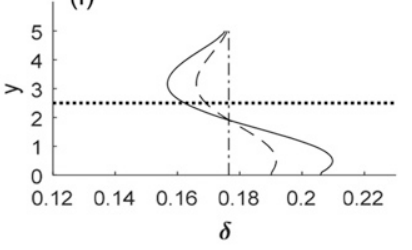

(h)

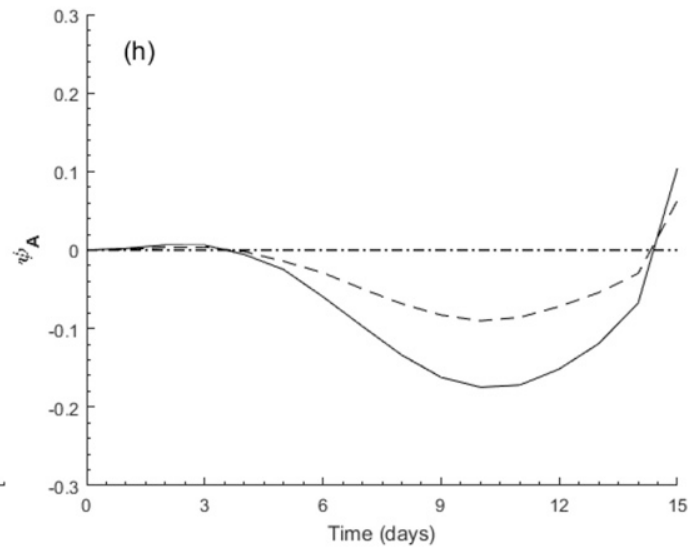

FIG. 17. (top) Variations of (a) $U$, (b) $\Delta \omega$, (c) $\mathrm{PV}_{y}$, (d) $C_{g}$, (e) $\lambda$, and (f) $\delta$ with $y$. (bottom) Time variations of the (g) blocking amplitude $\psi_{D}$ and $(\mathrm{h})$ asymmetry $\psi_{A}$ during the blocking cycle for three cases: $\Delta u=0$ (dot-dashed line), -0.1 (long-dashed line) and -0.2 (solid line) of $U=u_{0}+\Delta u e^{-\gamma\left(y-y_{0}\right)^{2}} \cos \left\{\left[2 \pi /\left(L_{y}+y_{1}\right)\right] y\right\}$ with $u_{0}=0.7$, $\gamma=0.1, y_{0}=1.5$, and $y_{1}=3$. The dotted line in (a)-(f) denotes the central latitude of $y=2.5$.

background zonal winds affect the lifetime, strength and north-south asymmetry of the subsequent eddy-driven dipole blocking. In this ENMI model, the atmospheric blocking may be understood as a nonlinear evolution of a large-scale Rossby wave packet described by a forced NLS equation under the forcing of preexisting synopticscale eddies. Results of this study show that the meridional distribution of the background zonal winds can significantly influence the north-south asymmetry, persistence, and amplitude of the eddy-driven dipole blocking through changing the meridional distributions of its movement speed, energy dispersion, and nonlinearity. It is shown that the meridional gradient of the potential vorticity $\left(\mathrm{PV}_{y} \approx\right.$ $\beta-U_{y y}+F U$ ), which combines the effects of background zonal wind and its nonuniform meridional shear, is a better metric than the zonal wind $U$ itself for quantifying the influence of the background zonal wind on an eddydriven dipole blocking. Moreover, the blocking system satisfies the linear energy dispersion-nonlinearity strength inverse relation rule. A low- $\mathrm{PV}_{y}$ environment has low energy dispersion, strong nonlinearity, and long-lived eddy forcing especially in high latitudes and in weak background westerly wind regions, which leads to a long lifetime and a large amplitude for the eddy-driven dipole blocking. In this extended NMI model, the energy dispersion and nonlinearity strength of the blocking system is mainly associated with $\mathrm{PV}_{y}$, while its movement speed is influenced by the background zonal wind, $\mathrm{PV}_{y}$, and the blocking amplitude. Under some certain assumptions, dipole blocking with a longer lifetime or weaker energy 


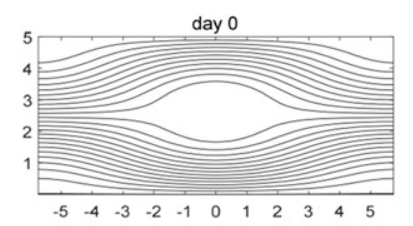

day 6

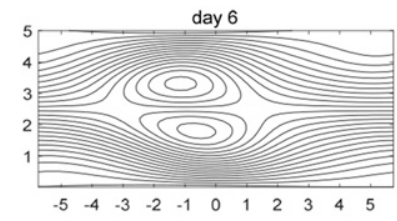

day 12

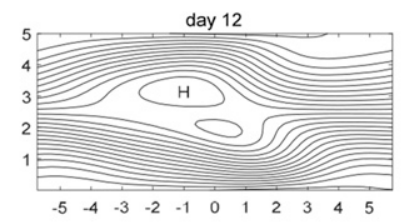

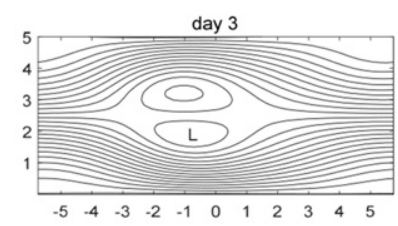

day 9

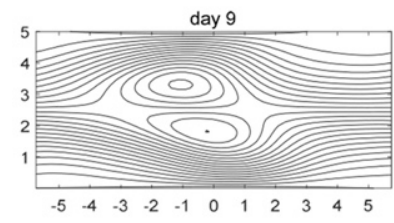

day 15

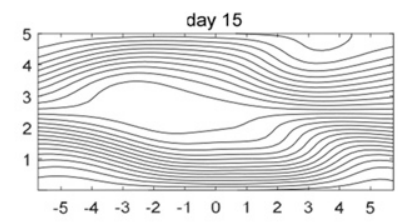

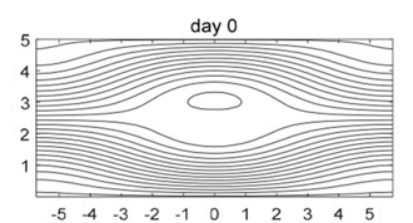

day 6

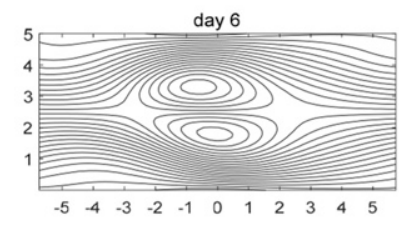

day 12

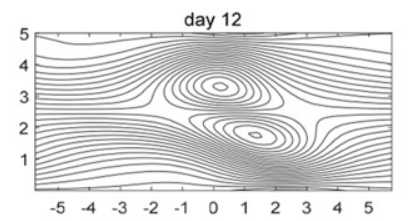

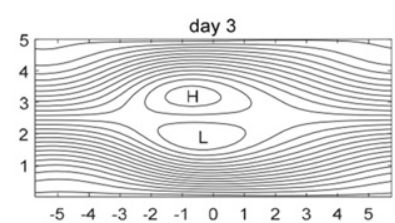

day 9

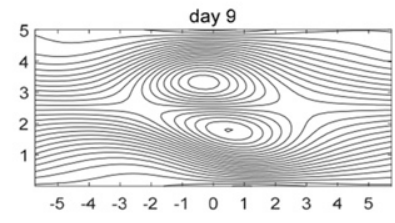

day 15

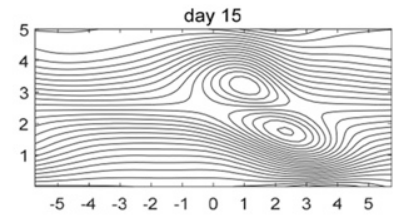

(a)

(b)

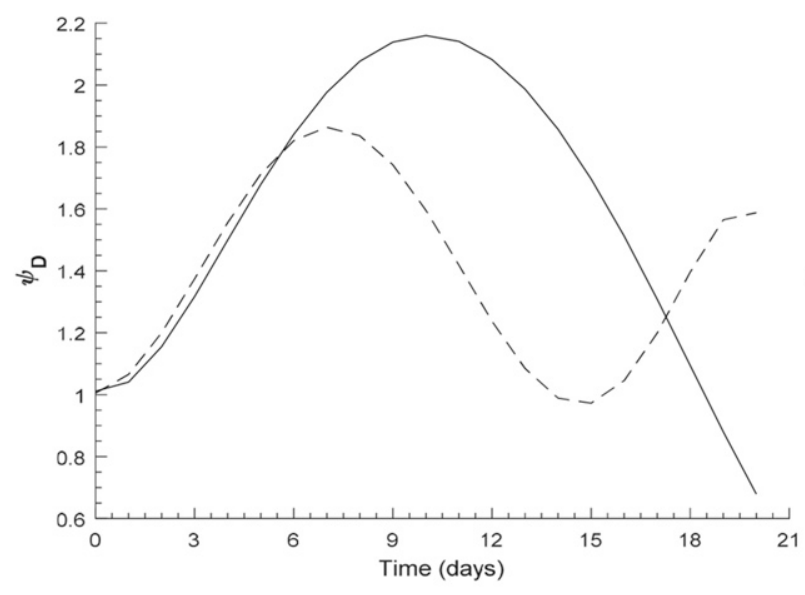

(c)

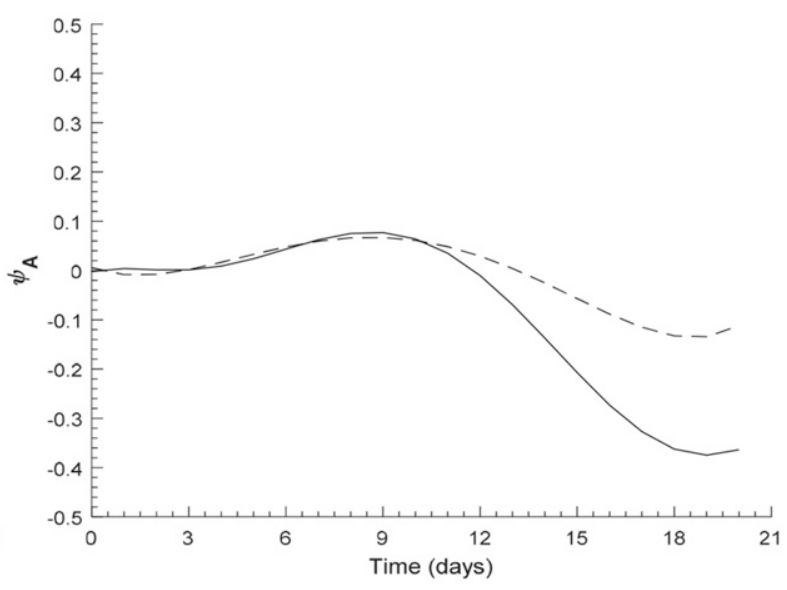

(d)

FIG. 18. (top) Instantaneous fields of planetary-scale streamfunction $\psi_{P}(\mathrm{CI}=0.15)$ during the life cycle of an eddy-driven dipole blocking for (a) $\mathrm{PV}_{y}=\beta+F U$ and (b) $\mathrm{PV}_{y}=\beta-U_{y y}$ with $U=u_{0}+\Delta u e^{-\gamma\left(y-y_{0}\right)^{2}} \cos \left\{\left[2 \pi /\left(L_{y}+y_{1}\right)\right] y\right\}, u_{0}=0.7, \Delta u=0.2, \gamma=0.1, y_{0}=1.5$, and $y_{1}=3$. (bottom) Temporal variations of the (c) amplitude $\psi_{D}$ and (d) asymmetry $\psi_{A}$ of an eddy-driven dipole blocking during its life cycle for $\mathrm{PV}_{y}=\beta+F U$ (dashed line) and $\mathrm{PV}_{y}=\beta-U_{y y}$ (solid line).

dispersion requires that the blocking amplitude cannot be too large or the background $\mathrm{PV}_{y}$ cannot be too small.

When the background jet has a double-branch structure, the lifetime of blocking is lengthened compared to the effect of uniform westerly winds. Especially, when the core of the background jet shifts from higher to lower latitudes, the energy dispersion (nonlinearity) of the blocking dipole is weakened (intensified) because of reduced PV gradient in higher latitudes. In this case, the northern pole of the dipole blocking can be maintained and undergo an accelerated westward movement because of its large amplitude and the weak westerly wind. In contrast, the energy dispersion (nonlinearity) of the lower-latitude part of the blocking system is intensified (reduced) because of increased $\mathrm{PV}_{\mathrm{y}}$, so that the southern pole disperses its energy rapidly and has small amplitude. As a result, the blocking dipole inevitably shows a strong NW-SE-oriented asymmetric dipole with a strong anticyclonic anomaly to the northwest and a weak cyclonic anomaly to the southeast. However, a strong NE-SW-oriented asymmetric dipole blocking with a weak anticyclonic anomaly to the northeast and a strong cyclonic anomaly to the southwest is seen when the background jet shifts from lower to higher latitudes. The NW-SE (NE-SW) orientation of this asymmetric 
blocking dipole can be explained in terms of the meridional profile of the nonlinear phase speed (the moving speed) of the dipole blocking, since the nonlinear phase speed is related to the meridional distributions of the background zonal wind, $\mathrm{PV}_{y}$ and the blocking amplitude. While the meridional distribution of the background zonal wind can significantly influence the meridional asymmetry of the blocking dipole, the magnitude of the meridionally averaged $\mathrm{PV}_{y}$, which is related to the background zonal winds, is crucial for the lifetime and strength of the dipole blocking. We also found that in $\mathrm{PV}_{y} \approx \beta-U_{y y}+F U$ the nonuniform meridional shear of the background zonal winds $U_{y y}$ is more important for the intensity, movement, and lifetime of the dipole blocking than the weakened background zonal wind strength $U$. These are new findings not shown by previous studies, including Luo et al. (2014). Of course, based on the magnitude and spatial distribution of $\mathrm{PV}_{y}$, we may design an index to describe how the background condition affects the duration and movement of blocking. This provides a useful tool for examining how Arctic warming influences atmospheric blocking and midlatitude cold extremes through changing the background westerly wind and $\mathrm{PV}_{y}$ in the mid- to high latitudes.

It must be pointed out that in this extended NMI model, we have neglected the nonuniform distribution of the background zonal winds in the zonal direction and the role of background meridional wind. If the zonal nonuniformity of the PV gradient is considered, the extended
NMI model may be used to explain why teleconnection wave trains such as the Pacific-North American (PNA) pattern are often formed in the North Pacific and North America, but a localized dipole mode such as the North Atlantic Oscillation (NAO) is usually generated in the North Atlantic. This problem will be further investigated in the future study.

Acknowledgments. The authors acknowledge the support from the National Natural Science Foundation of China (Grant 41430533) and the National Key Research and Development Program of China (2016YFA0601802). Dai acknowledges the funding support from the U.S. National Science Foundation (Grants AGS-1353740 and OISE-1743738), the U.S. Department of Energy's Office of Science (Award DE-SC0012602), and the U.S. National Oceanic and Atmospheric Administration (Award NA15OAR4310086). The authors thank the three anonymous reviewers for their constructive comments.

\section{APPENDIX A}

\section{Coefficients of Deformed Eddies in Eq. (2g)}

The following equations define the coefficients of deformed eddies in Eq. (2g).

$$
\begin{aligned}
& p_{j}=\frac{k-2 \tilde{k}_{j}}{\operatorname{PV}_{y}\left\{\tilde{k}_{j}+k-\left(\frac{\tilde{k}_{j}}{\tilde{k}_{j}^{2}+m^{2} / 4+F}+\frac{k}{k^{2}+m^{2}+F}\right)\left[\left(\tilde{k}_{j}+k\right)^{2}+9 m^{2} / 4+F\right]\right\}}, \\
& r_{j}=\frac{k+2 \tilde{k}_{j}}{\operatorname{PV}_{y}\left\{\tilde{k}_{j}+k-\left(\frac{\tilde{k}_{j}}{\tilde{k}_{j}^{2}+m^{2} / 4+F}+\frac{k}{k^{2}+m^{2}+F}\right)\left[\left(\tilde{k}_{j}+k\right)^{2}+m^{2} / 4+F\right]\right\}}, \\
& s_{j}=\frac{k+2 \tilde{k}_{j}}{\operatorname{PV}_{y}\left\{\tilde{k}_{j}-k-\left(\frac{\tilde{k}_{j}}{\tilde{k}_{j}^{2}+m^{2} / 4+F}-\frac{k}{k^{2}+m^{2}+F}\right)\left[\left(\tilde{k}_{j}-k\right)^{2}+9 m^{2} / 4+F\right]\right\}}, \\
& h_{j}=\frac{k-2 \tilde{k}_{j}}{\mathrm{PV}_{y}\left\{\tilde{k}_{j}-k-\left(\frac{\tilde{k}_{j}}{\tilde{k}_{j}^{2}+m^{2} / 4+F}-\frac{k}{k^{2}+m^{2}+F}\right)\left[\left(\tilde{k}_{j}-k\right)^{2}+m^{2} / 4+F\right]\right\}},
\end{aligned}
$$




$$
Q_{j}=k^{2}+m^{2}-\left(\tilde{k}_{j}^{2}+m^{2} / 4\right), \quad j=1,2 .
$$

\section{APPENDIX B}

\section{Coefficients of the Nonlinearity Strength and Preexisting Eddy Forcing in Eq. (2h)}

The following equations define the coefficients of the nonlinearity strength and preexisting eddy forcing in Eq. (2h).

$$
\begin{aligned}
& \delta_{N}=\frac{k m \sum_{n=1}^{\infty} q_{N n} g_{n}^{2}\left[k^{2}+m^{2}-m^{2}(n+1 / 2)^{2}\right]}{k^{2}+m^{2}+F}, \\
& q_{N n}=\frac{4 k^{2} m}{L_{y}\left\{1-\left(m^{2}+F-k^{2}\right)\left[F+m^{2}(n+1 / 2)^{2}\right] /\left(k^{2}+m^{2}+F\right)^{2}\right\}} \text {, } \\
& g_{n}=\frac{8}{m\left[4-(n+1 / 2)^{2}\right] L_{y}},
\end{aligned}
$$

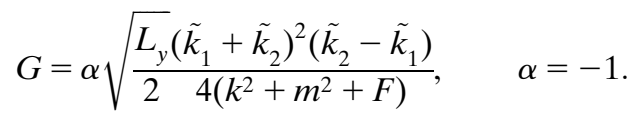

\section{REFERENCES}

Berggren, R., B. Bolin, and C.-G. Rossby, 1949: An aerological study of zonal motion, its perturbations and break-down. Tellus, 1 (2), 14-37, https://doi.org/10.3402/tellusa.v1i2.8501.

Charney, J. G., and J. G. DeVore, 1979: Multiple flow equilibria in the atmosphere and blocking. J. Atmos. Sci., 36, 1205-1216, https://doi.org/10.1175/1520-0469(1979)036<1205: MFEITA $>2.0 . \mathrm{CO} ; 2$.

Colucci, S. J., 1985: Explosive cyclogenesis and large-scale circulation changes: Implications for atmospheric blocking. J. Atmos. Sci., 42, 2701-2717, https://doi.org/10.1175/ 1520-0469(1985)042<2701:ECALSC > 2.0.CO;2.

Dai, A., D. Luo, M. Song, and J. Liu, 2019: Arctic amplification is caused by sea-ice loss under increasing $\mathrm{CO}_{2}$. Nat. Commun., 10, 121, https://doi.org/10.1038/s41467-018-07954-9.

Diao, Y., J. Li, and D. Luo, 2006: A new blocking index and its application: Blocking action in the Northern Hemisphere. J. Climate, 19, 4819-4839, https://doi.org/10.1175/JCLI3886.1.

Dole, R. M., and N. D. Gordon, 1983: Persistent anomalies of the extratropical Northern Hemisphere wintertime circulation: Geographical distribution and regional persistence characteristics. Mon. Wea. Rev., 111, 1567-1586, https://doi.org/ 10.1175/1520-0493(1983)111<1567:PAOTEN > 2.0.CO;2.

— , and Coauthors, 2011: Was there a basis for anticipating the 2010 Russian heat wave? Geophys. Res. Lett., 38, L06702, https://doi.org/10.1029/2010GL046582.

Egger, J., 1978: Dynamics of blocking high. J. Atmos. Sci., 35, 1788-1801, https://doi.org/10.1175/1520-0469(1978)035<1788: $\mathrm{DOBH}>2.0 . \mathrm{CO} ; 2$.

Haines, K., and J. C. Marshall, 1987: Eddy-forced coherent structures as a prototype of atmospheric blocking. Quart. J. Roy. Meteor. Soc., 113, 681-704, https://doi.org/10.1002/qj.49711347613.

—_, and A. J. Holland, 1998: Vacillation cycles and blocking in a channel. Quart. J. Roy. Meteor. Soc., 124, 873-895, https:// doi.org/10.1002/qj.49712454711.
Holopainen, E., and C. Fortelius, 1987: High-frequency transient eddies and blocking. J. Atmos. Sci., 44, 1632-1645, https://doi.org/ 10.1175/1520-0469(1987)044<1632:HFTEAB >2.0.CO;2.

Hoskins, B. J., I. N. James, and G. H. White, 1983: The shape, propagation and mean-flow interaction of large-scale weather systems. J. Atmos. Sci., 40, 1595-1612, https://doi.org/10.1175/ 1520-0469(1983)040<1595:TSPAMF>2.0.CO;2.

Illari, L., and J. C. Marshall, 1983: On the interpretation of eddy fluxes during a blocking episode. J. Atmos. Sci., 40, 2232-2242, https://doi.org/10.1175/1520-0469(1983)040<2232: OTIOEF $>2.0 . \mathrm{CO} ; 2$.

Ji, L. R., and S. Tibaldi, 1983: Numerical simulations of a case of blocking: The effects of orography and land-sea contrast. Mon. Wea. Rev., 111, 2068-2086, https://doi.org/10.1175/ 1520-0493(1983)111<2068:NSOACO>2.0.CO;2.

Kitano, Y., and T. J. Yamada, 2016: Relationship between atmospheric blocking and cold day extremes in current and RCP8.5 future climate conditions over Japan and the surrounding area. Atmos. Sci. Lett., 17, 616-622, https://doi.org/ 10.1002/asl.711.

Lejenäs, H., and H. Økland, 1983: Characteristics of Northern Hemisphere blocking as determined from a long time series of observed data. Tellus, 35A, 350-362, https://doi.org/10.1111/ j.1600-0870.1983.tb00210.x.

Luo, D., 2000: Planetary-scale baroclinic envelope Rossby solitons in a two-layer model and their interaction with synopticscale eddies. Dyn. Atmos. Oceans, 32, 27-74, https://doi.org/ 10.1016/S0377-0265(99)00018-4.

_ 2005: A barotropic envelope Rossby soliton model for blockeddy interaction. Part I: Effect of topography. J. Atmos. Sci., 62, 5-21, https://doi.org/10.1175/1186.1.

— and synoptic-scale waves during the life cycle of blockings. Adv. Atmos. Sci., 17, 649-670, https://doi.org/10.1007/ s00376-000-0026-5.

- F. Huang, and Y. Diao, 2001: Interaction between antecedent planetary-scale envelope soliton blocking anticyclone and synoptic-scale eddies: Observations and theory. J. Geophys. Res., 106, 31795-31816, https://doi.org/10.1029/ 2000JD000086.

_ J. Cha, L. Zhong, and A. Dai, 2014: A nonlinear multiscale interaction model for atmospheric blocking: The eddyblocking matching mechanism. Quart. J. Roy. Meteor. Soc., 140, 1785-1808, https://doi.org/10.1002/qj.2337.

_ , Y. Xiao, Y. Yao, A. Dai, I. Simmonds, and C. L. E. Franzke, 2016: Impact of Ural blocking on winter warm Arctic-cold Eurasian anomalies. Part I: Blocking-induced amplification. J. Climate, 29, 3925-3947, https://doi.org/10.1175/JCLI-D-150611.1.

— X. Chen, A. Dai, and I. Simmonds, 2018: Changes in atmospheric blocking circulations linked with winter Arctic warming: A new perspective. J. Climate, 31, 7661-7678, https:// doi.org/10.1175/JCLI-D-18-0040.1.

McWilliams, J. C., 1980: An application of equivalent modons to atmospheric blocking. Dyn. Atmos. Oceans, 5, 43-66, https:/ doi.org/10.1016/0377-0265(80)90010-X.

Mu, M., and Z. Jiang, 2008: A method to find perturbations that trigger blocking onset: Conditional nonlinear optimal perturbations. J. Atmos. Sci., 65, 3935-3946, https://doi.org/10.1175/ 2008JAS2621.1.

Mullen, S. L., 1987: Transient eddy forcing of blocking flows. J. Atmos. Sci., 44, 3-22, https://doi.org/10.1175/1520-0469(1987) 044<0003:TEFOBF $>2.0$. CO;2. 
Muslu, G. M., and H. A. Erbay, 2005: Higher-order split-step Fourier schemes for the generalized nonlinear Schrödinger equation. Math. Comput. Simul., 67, 581-595, https://doi.org/ 10.1016/j.matcom.2004.08.002.

Nakamura, H., and J. M. Wallace, 1993: Synoptic behavior of baroclinic eddies during the blocking onset. Mon. Wea. Rev., 121, 1892-1903, https://doi.org/10.1175/1520-0493(1993) $121<1892:$ SBOBED $>2.0 . \mathrm{CO} ; 2$.

Nakamura, N., and S. Y. Huang, 2018: Atmospheric blocking as a traffic jam in the jet stream. Science, 361, 42-47, https://doi.org/ 10.1126/science.aat0721.

Nayfeh, A. H., 2000: Perturbation Methods. Wiley, 425 pp.

Ogi, M., K. Yamazaki, and Y. Tachibana, 2004: The summertime annular mode in the Northern Hemisphere and its linkage to the winter mode. J. Geophys. Res., 109, D20114, https:// doi.org/10.1029/2004JD004514.

Pelly, J. L., and B. J. Hoskins, 2003: A new perspective on blocking. J. Atmos. Sci., 60, 743-755, https://doi.org/10.1175/ 1520-0469(2003)060<0743:ANPOB >2.0.CO;2.

Rex, D. F., 1950: Blocking action in the middle troposphere and its effect upon regional climate. I: An aerological study of blocking action. Tellus, 2, 196-211, https://doi.org/10.3402/ tellusa.v2i3.8546.

Shukla, J., and K. C. Mo, 1983: Seasonal and geographical variation of blocking. Mon. Wea. Rev., 111, 388-402, https://doi.org/ 10.1175/1520-0493(1983)111<0388:SAGVOB >2.0.CO;2.
Shutts, G. J., 1983: The propagation of eddies in diffluent jetstreams: Eddy vorticity forcing of blocking flow fields. Quart. J. Roy. Meteor. Soc., 109, 737-761, https://doi.org/10.1002/ qi.49710946204.

Tachibana, Y., T. Nakamura, H. Komiya, and M. Takahashi, 2010: Abrupt evolution of the summer Northern Hemisphere annular mode and its association with blocking. J. Geophys. Res., 115, D12125, https://doi.org/10.1029/2009JD012894.

Thorncroft, C. D., B. J. Hoskins, and M. E. McIntyre, 1993: Two paradigms of baroclinic wave life-cycle behavior. Quart. J. Roy. Meteor. Soc., 119, 17-55, https://doi.org/10.1002/qj.49711950903.

Tibaldi, S., and F. Molteni, 1990: On the operational predictability of blocking. Tellus, 42A, 343-365, https://doi.org/10.3402/ tellusa.v42i3.11882.

Tung, K. K., and R. S. Lindzen, 1979: A theory of stationary long waves. Part I: A simple theory of blocking. Mon. Wea. Rev., 107, 714-734, https://doi.org/10.1175/1520-0493(1979) $107<0714$ :ATOSLW $>2.0$.CO;2.

Yao, Y., D. Luo, A. Dai, and I. Simmonds, 2017: Increased quasi stationarity and persistence of Ural blocking and Eurasian extreme cold events in response to Arctic warming. Part I: Insight from observational analyses. J. Climate, 30, 3549-3568, https://doi.org/10.1175/JCLI-D-16-0261.1.

Yeh, T. C., 1949: On energy dispersion in the atmosphere. J. Meteor., 6, 1-16, https://doi.org/10.1175/1520-0469(1949) $006<0001$ :OEDITA $>2.0 . \mathrm{CO} ; 2$ 


\title{
CORRIGENDUM
}

\author{
Dehai LuO ${ }^{\mathrm{a}, \mathrm{b}}$ \\ ${ }^{a}$ CAS Key Laboratory of Regional Climate-Environment for Temperate East Asia, Chinese Academy of Science, Beijing, China \\ ${ }^{\mathrm{b}}$ University of Chinese Academy of Sciences, Beijing, China
}

(Manuscript received 27 March 2020, in final form 20 May 2021)

KEYWORDS: Blocking; Nonlinear dynamics; Potential vorticity

There is an incorrect writing in the equation of $G$ in appendix B in Luo et al. (2019) and also in Luo et al. (2018) because of a typographic error, although the results are not affected. The equation of $G$ should read

$$
G=\alpha \sqrt{\frac{L_{y}}{2}} \frac{\left(\tilde{k}_{1}+\tilde{k}_{2}\right)^{2}\left(\tilde{k}_{2}-\tilde{k}_{1}\right) m}{4\left(k^{2}+m^{2}+F\right)} .
$$

The authors regret any inconvenience this error may have caused.

\section{REFERENCES}

Luo, D., X. Chen, and S. Feldstein, 2018: Linear and nonlinear dynamics of North Atlantic Oscillations: A new thinking of symmetry breaking. J. Atmos. Sci., 75, 1955-1977, https://doi.org/10.1175/JAS-D-170274.1.

— W. Zhang, L. Zhong, and A. Dai, 2019: A nonlinear theory of atmospheric blocking: A potential vorticity gradient view. J. Atmos. Sci., 76, 2399-2427, https://doi.org/10.1175/JAS-D-18-0324.1. 